\title{
A Sampling Design for the 1980 Commercial and Multifamily Residential Building Survey
}

W.M. Bowen

A. R. Olsen

A. L. Nieves

June 1981

Prepared for the U.S. Department of Energy under Contract DE-AC06-76RLO 1830

Pacific Northwest Laboratory

Operated for the U.S. Department of Energy by Battelle Memorial Institute 


\title{
NOTICE
}

This report was prepared as an account of work sponsored by the United States Government. Neither the United States nor the Department of Energy, nor any of their employees, nor any of their contractors, subcontractors, or their employees, makes any warranty, express or implied, or assumes any legal liability or responsibility for the accuracy, completeness or usefulness of any information, apparatus, product or process disclosed, or represents that its use would not infringe privately owned rights.

The views, opinions and conclusions contained in this report are those of the contractor and do not necessarily represent those of the United States Government or the United States Department of Energy.

\author{
PACIFIC NORTHWEST LABORATORY \\ operated by \\ BATTELLE \\ for the \\ UNITED STATES DEPARTMENT OF ENERGY \\ Under Contract DE-AC06-76RLO 1830
}

\author{
Printed in the United States of America \\ Available from \\ National Technical Information Service \\ United States Department of Commerce \\ 5285 Port Royal Road \\ Springfield, Virginia 22151
}

Price: Printed Copy $\$$ $\therefore$ Microfiche $\$ 3.00$

NTIS
-Pages Selling Price

001-025 $\$ 4,00$

026-050 \$4.50

051-075 \$5.25

076-100 $\$ 5.00$

$101-125 \quad \$ 6.50$

$126-150 \quad \$ 7.25$

$151-175 \quad \$ 8.00$

$176-200 \quad \$ 9.00$

$201-225 \quad \$ 9.25$

$226-250 \quad \$ 9.50$

$251-275 \quad \$ 10.75$

276-300 $\$ 11.00$ 
PNL-3883

UC -32

33679000597882

A SAMPLING DESIGN FOR THE 1980 COMMERCIAL AND MULTIFAMILY RESIDENTIAL BUILDING SURVEY

W.M. Bowen

A.R. 01 sen

A.L. Nieves

June 1981

Prepared for

the U.S. Department of Energy

under Contract DE-AC06-76RLO 1830

Pacific Northwest Laboratory

Richland, Washington 99352 
-

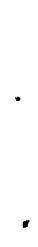




\section{CONTENTS}

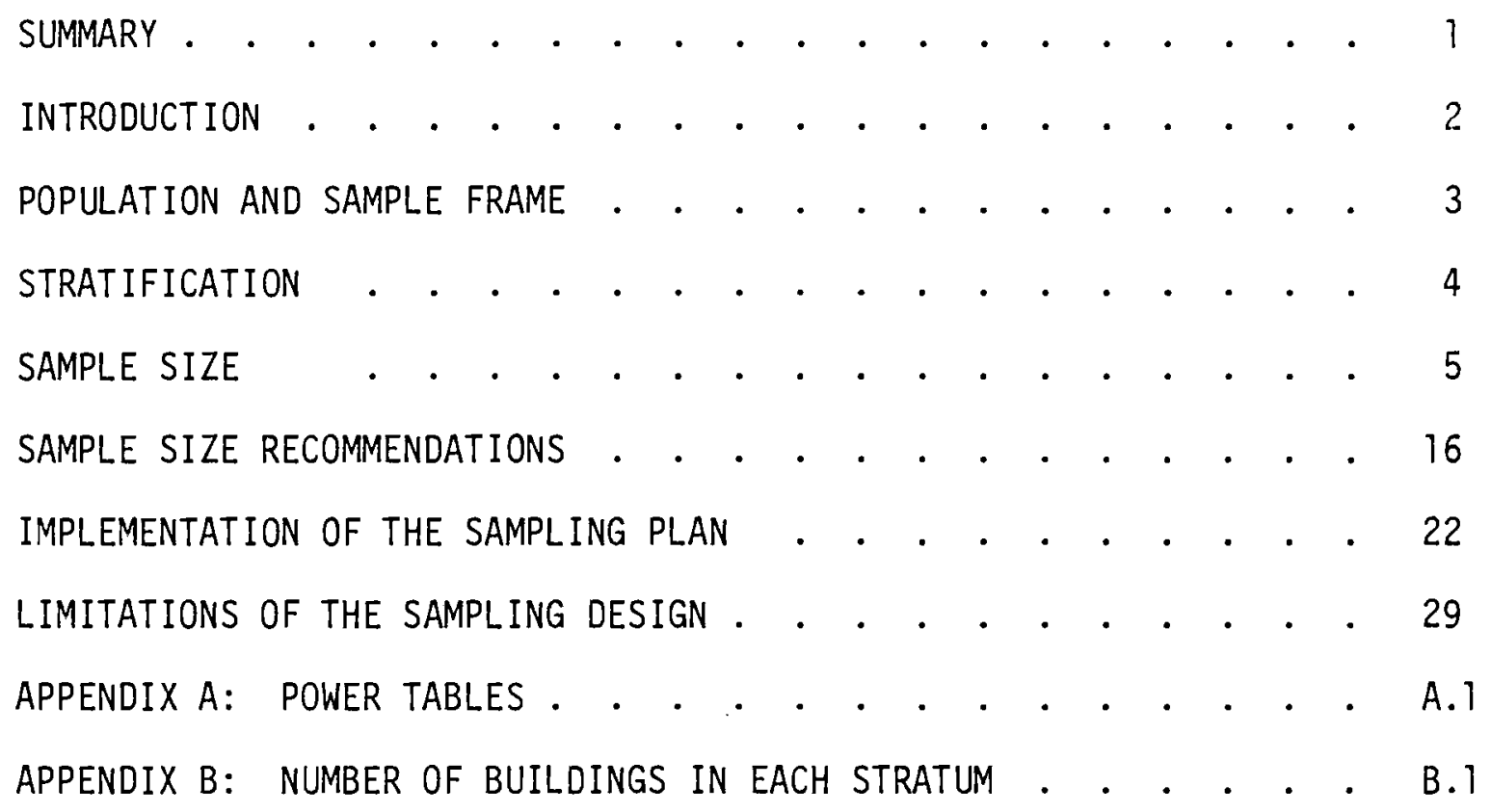


A sampling plan is proposed for the 1980 Conmercial and Multifamily Building Energy Performance Survey. The sampling plan is designed to acquire sufficient information to make inferences about nationwide compliance with $\mathrm{DEB}_{80}$. Due to lack of information about the per building costs associated with conducting the survey, several alternative sample sizes are discussed. Implementation of the sampling plan is described, and formulas are given for the statistical analysis of the survey data. 


\section{INTRODUCTION}

This report presents details of a proposed sample design for the 1980 Commercial and Multifamily Building Energy Performance Survey. The objective of the survey is to assess the extent to which new building design practices comply with the proposed "1980 Energy Budget Levels for Commercial and Multifamily Residential Building Designs" $\left(\mathrm{DEB}_{80}\right)$. The procedure will be to:

(1) identify a small number of building types which account for the majority of commercial buildings constructed in the U.S.A.;

(2) conduct a "separate survey" for each building type;

(3) include only buildings designed during 1980 .

For each building in the survey, the Design Energy Consumption $\left(D C_{80}\right)$ will be determined by the DOE2.1 computer program. The quantity

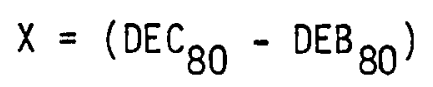

will be calculated for each building as a measure of its compliance with ${ }^{D E B} 80^{\circ}$. These $X$ quantities will then be used to compute sample statistics. Inferences about nationwide compliance with $D^{D E B} 80$ may then be made for each building type. Subsequent sections of this report provide details of the population, sampling frame, stratification, sample size, and implementation of the sampling plan. Although there are additional objectives of the study, this sample design addresses only the primary objective described above. 


\section{POPULATION AND SAMPLE FRAME}

The population of interest includes all commercial and multifamily residential buildings in the U.S.A. for which designs were completed during calendar year 1980. Energy requirements (and indeed ${ }^{D E B} 80$ 's) differ by building type. For the purpose of this survey, each building type is viewed as a subpopulation. Since it is infeasible, in terms of money and resources, to include all building types in the survey the scope of the survey is narrowed by excluding:

1. buildings valued at less than $\$ 100,000$

2. additions or alterations

3. church, YMCA, bowling alley, club, lodge, studio, exhibition hall, laboratory, government, manufacturing, or restaurant buildings.

The building types included in the survey (funding permitting) and their corresponding F.W. Dodge Structure Codes (DSC) are listed in Table 1 in descending order of importance to the survey. It is recommended that the survey proceed by building type in the order suggested by Table 1 . After the surveys have been completed for the high priority building types, should funding limitation preclude sampling from all building types, the following options may then be exercised:

1. sparse sampling of some or all remaining building types (this may involve collapsing geographic regions and is discussed in subsequent sections), or

2. complete sampling of as many of the remaining building types as funding permits, i.e., eliminate some of the lower priority building types from the study. 
For each building type the sample frame consists of all buildings and construction starts listed on the $1980 \mathrm{~F} . W$. Dodge tapes for which construction plans are available. The total number of buildings listed on the $1980 \mathrm{~F} . \mathrm{W}$. Dodge tapes for each building type is denoted $K_{80}$ and is displayed in Table 1. The actual number of these buildings for which construction plans are available is unknown, but it is believed that construction plans are available for most (say 95\%) of the $k_{80}$ buildings of each type. 
TABLE 1. Building Types to be Included in the -urvey with Dodge Structure Code, Number of Buildings listed on the 1980 F.W. Dodge Tapes and Standard Deviation of $\mathrm{DEC}_{76}-\mathrm{DEB}_{80}$

$\begin{array}{lcrr}\text { Building Type } & \underline{\text { DSC }} & \underline{K_{80}} & \underline{S_{76}} \\ \text { 1. Large Office* } & 5,6,7 & 1039 & 17.87 \\ \text { 2. Small Office+ } & 5,6,7 & 9400 & 25.17 \\ \text { 3. Low-rise Apartments } & 70,75 & 13253 & 18.55 \\ \text { 4. High-rise Apartments } & 71 & 1115 & 18.55 \\ \text { 5. Elementary Schoo } & 41 & 371 & 16.44 \\ \text { 6. Secondary Schoo } 1 & 42,43,44,45 & 596 & 28.80 \\ \text { 7. Clinic } & 94 & 1702 & 40.32 \\ \text { 8. Hospital } & 93 & 144 & 109.22 \\ \text { 9. Nursing Home } & 95 & 19 & 39.50 \\ \text { 10. Store } & 1 & 5474 & 31.05 \\ \text { 11. Shopping Center } & 101 & 1206 & 31.16 \\ \text { 12. Warehouse. } & 3 & 8048 & 19.56 \\ \text { 13. Hotel/Motel } & 69,72,73 & 513 & 17.30 \\ \text { 14. Theater/Auditorium } & 56,59,256 & 178 & 23.22 \\ \text { 15. Community Center } & 63,65 & 223 & 22.13 \\ \text { 16. Gymnasium } & 62,262 & 578 & 35.31\end{array}$

* floor area at least 50K $\mathrm{ft}^{2}$

+ floor area less than 50K $\mathrm{ft}^{2}$ 


\section{STRATIFICATION}

The United States, including Alaska and Hawaii, is stratified by $c l$ imate zone and Census Division. Seven climate zones were defined for the 1976 AIA/RC Phase II Study in terms of the number of heating and cooling degree days. Weather data were then used to assign each county in the U.S.A. to its appropriate climate zone, see Figure 1 . The fifty states are assigned to the four Census Regions* which are further divided into nine Census Divisions, see Figure 1. Thus, the stratification of the U.S.A. is defined by:

\section{States assigned to Census Divisions}

2. Counties within Census Divisions assigned to climate zones (see Figure 1)

This stratification of Census Divisions by $\mathrm{climate}$ zones implies a total of sixty-three strata. However, Table 2 reveals that there are actualiy thirtyeight strata.

An important goal of the stratification is to ensure that all climate zones in every Census Division are represented in the survey. However, in certain strata, there were no 1980 designs for some building types. Thus, it will be necessary to judiciously collapse similar strata, while attempting to maintain a fair representation of geographic regions and climate zones in the survey. The necessary information for such decisions, presented in Appendix $B$, comes from a summary analysis of the $1980 \mathrm{~F}$.W. Dodge tapes.

* See, e.g., "Characteristics of New Housing: 1979", Construction Reports C2579-13, U.S. Department of Commerce, Bureau of the Census, page 69. 


\section{CLIMATE ZONES}

1. \|\|$^{m}||-2000$ CDD

- $>7000 \mathrm{HDD}$

2. $\square \begin{aligned} & <2000 \text { CDD } \\ & 5000-7000 \text { HDD }\end{aligned}$

3. $<2000 \mathrm{CDD}$ 4000-5500 HDD

4. $य \begin{aligned} & 2000 \text { CDD } \\ & 2000-4000 \text { HDD }\end{aligned}$

5. $<2000$ CDD

6. $>2000 \mathrm{CDD}$ 2000 CDD
$0-2000$ HDD

7.
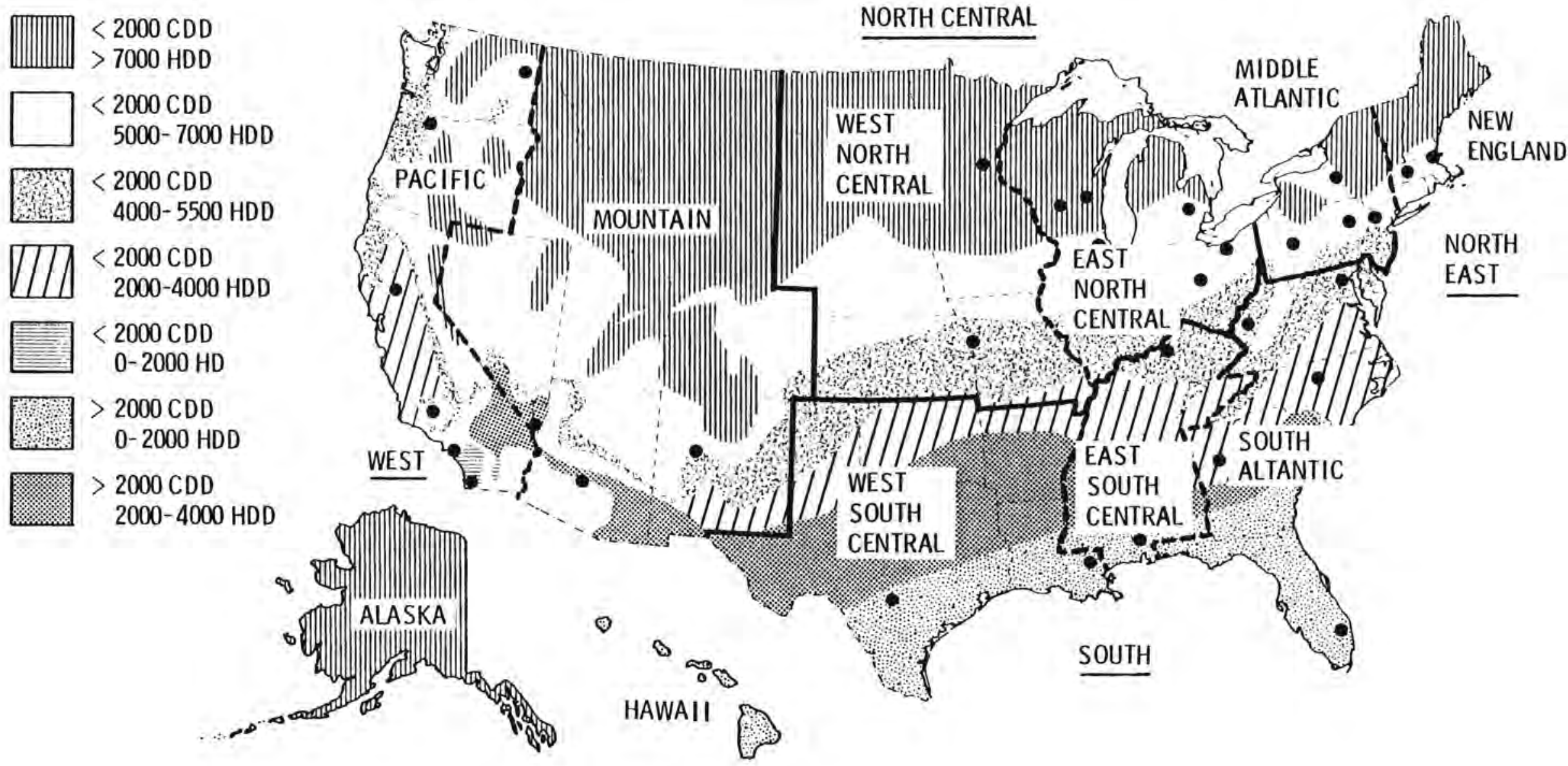

FIGURE 1 CLIMATE ZONES DEFINED BY THE AIA/RC WITH CENSUS REGIONS AND DIVISIONS 
TABLE 2. Contingency Table Showing Presence of Climate Zones in Census Division. There Are Thirty-Eight Strata

CENSUS DIVISION

AIA/RC PHASE II CLIMATE ZONES TOTAL STRATA

\begin{tabular}{lllllll}
1 & 2 & 3 & 4 & 5 & 6 & 7 \\
\hline
\end{tabular}

New Engl and

(NE) $\quad x \quad x$

Mid-Atlantic (NE) $x \quad x \quad x$

So. Atlantic

(S) $x \times x \times$

$x \quad x$

E. So. Central ( $S$ )

$x \quad x$

$x \quad x$

4

W. So. Central ( S)

$x \quad x$

$x \quad x$

4

E. No. Central (NC) $x \times x$

W. No. Centrat (NC) $x \quad x \quad x \quad x$

4

Mountain

(W) $x \times x \times$

$x \quad x$

6

Pacific

$x \quad x$

$x \quad x^{*} \quad x \quad x$

6

TOTAL

* Climate Zones 4 and 5 were collapsed leaving a total of 38 strata 


\section{SAMPLE SIZE}

In order to determine sample size it is necessary to establish:

(1) The desired precision of parameter estimates;

(2) The power of the hypothes is test(s).

These, however, are administrative and management decisions rather than statistical perspectives. Both, however, will influence the sample sizes necessary for this survey. In any event, some knowledge of the variability existing within the population is essential. The measure of concern for each of the building types is $\mathrm{DEC}_{80}-\mathrm{DEB}_{80}$. Estimates of the standard deviation for each building type are calculated from data collected in Phase II of the 1976 AIA/RC Commercial Building Survey. These estimates (see Table 1) are used as approximations of the standard deviations of $\mathrm{DEC}_{80}-\mathrm{DEB}_{80}$. For purposes of this sample design, in other words, is is assumed that

Std. Dev. of $\left(D E C_{76}-D^{D E B} 80\right)=$ Std. Dev. of $\left(D E C_{80}-D E B_{80}\right)$.

An objective of the survey for each building type is to be able to construct a $100(1-\alpha) \%$ confidence interval for the 80th percentile of the distribution of $x=\left(D E C_{80}-D E B_{86}\right)$ such that the half-width of the interval is no more than $5 \mathrm{MBtu} / \mathrm{ft}^{2} /$ year. That is, it is desirable that an estimate of the 80th percentile be correct to within $\pm 5 \mathrm{MBtu}$ of the true value of the 80 th percentile, say $x_{.80}$, in the sense that if the sample estimate is $\hat{x}_{.80}=-20 \mathrm{MBtu}$ there is a $100(1-\alpha) \%$ chance that the true value, $x_{.80}$, is between $-25 \mathrm{MBtu}$ and $-15 \mathrm{MBtu}$. In this manner, we would interpret $\hat{X}_{8}=20 \mathrm{MBtu}$ to mean that it is estimated that 80 percent of the buildings within the type being examined have $D E C_{80}$ values that are at least 20 MBtu below their $\mathrm{DEB}_{80}$ values. Thus, if $\alpha=.05$, there exists a 95 percent probability that 80 percent of the buildings in the relevant type have $D^{2} C_{80}$ values at least $15 \mathrm{MB}$ tu to $25 \mathrm{MBt}$ below their $\mathrm{DEB}_{80}$ values. 
If it can be assumed that $X$ follows approximately a normal distribution with standard deviation $\sigma$, then the standard deviation of the estimator $\hat{x} .80$ is

Std. Dev. $\left(\hat{x}_{.80}\right)=\left(\frac{k_{80}-n}{n K_{80}}\right)\left(\frac{.40}{.2803}\right) \sigma$

where $n$ is the sample size. Assuming further that $\hat{x}_{.80}$ follows a normal distribution, the sample size required to construct a 100 (1- $\alpha) \%$ confidence interval for $X_{.80}$ with half-width 5 MBtu is given by

where

$$
n=n_{0} /\left(1+n_{0} / k_{80}\right),
$$

$$
n_{0}=\left[z_{\alpha / 2} \sigma(.4 / .2803) / 5\right]^{2}
$$

and where $Z \alpha / 2$ is the $\alpha / 2$ quantile of the standard normal distribution.

For each building type the $S_{76}$ value from Table 1 was used in place of $\sigma$ in equation (3) to obtain an approximation of the required sample size for a specified value of $\alpha$.

Using Large Office Buildings as an example, the value $S_{76}=17.87$ is obtained from Table 1. To approximate the sample sizes required to construct $80 \%, 90 \%$ and $95 \%$ confidence intervals for $x .80$, each having a half-width of $5 \mathrm{MBtu}$, the values $z_{.10}=-1.282, z_{.05}=-1.645$, and $z_{.025}=-1.96$, respectively, are substituted for $Z \alpha_{/ 2}$, and $S_{76}=17.87$ replaces $\sigma$ in equation (3). The resulting sample sizes are calculated as follows:

$$
n_{0}=[-1.282(17.87)(.2854)]^{2}=43
$$

and

$$
n=43 /(1+43 / 1039)=42
$$


for an $80 \%$ confidence interval;

$$
n_{0}=[-1.645(17.87)(.2854)]^{2}=71
$$

and

$$
\mathrm{n}=71 /(1+71 / 1039)=67
$$

for a $90 \%$ confidence interval; and

$$
\begin{aligned}
& n_{0}=[-1.96(17.87)(.2854)]^{2}=100 \\
& \text { and } n=100 /(1+100 / 1039)=92
\end{aligned}
$$

for a 95\% confidence interval. Sample sizes computed in this manner for each of the building types are displayed in Table 3.

It should be noted that the assumption that $x$ and $\hat{x}_{.80}$ follow normal distributions is probably not true. However, the sample sizes determined under this assumption are considered reasonable for practical purposes.

It can be seen from Table 3 that certain building types require quite large sample sizes to permit the construction of confidence intervals with halfwidths 5MBtu. This is due to the size of their $S_{76}$ values. It may be reasonable for some building types to require the half-widths of the confidence intervals for $x_{.80}$ to be some fraction of $\sigma$. Using this concept, equation (3) can be written

$$
n=n_{0} /\left(1+n_{0} / K_{80}\right)
$$

where

$$
n_{0}=\left[z_{\alpha / 2}(.4 / .2803) / C\right]^{2}
$$


TABLE 3. Required Sample Sizes for $100(1-\alpha) \%$ Confidence Intervals for $X_{.80}$ with Half-Width 5MBtu

\begin{tabular}{lrrr} 
& \multicolumn{3}{c}{$(1-\alpha)$} \\
\cline { 2 - 4 } Building Type & $80 \%$ & $90 \%$ & $95 \%$ \\
\hline Large Office & 42 & 67 & 92 \\
Smal1 Office & 85 & 140 & 199 \\
Low-rise Apartments & 46 & 76 & 108 \\
High-rise Apartments & 46 & 72 & 99 \\
Elementary School & 34 & 52 & 70 \\
Secondary School & 95 & 140 & 181 \\
Clinic & 194 & 297 & 392 \\
Hospital & 132 & 137 & 139 \\
Nursing Home & 18 & 18 & 19 \\
Store & 129 & 206 & 290 \\
Shopping Center & 118 & 182 & 243 \\
Warehouse & 52 & 85 & 120 \\
Hotel/Motel & 38 & 59 & 80 \\
Theater/Auditorium & 52 & 72 & 87 \\
Community Center & 51 & 73 & 92 \\
Gymnasium & 128 & 187 & 233 \\
\hline & & & \\
Total of Sample Sizes & 1260 & 1863 & 2444
\end{tabular}


and where $C$ is some fraction. Equation (4) requires no knowledge of $\sigma$. The interpretation here is that if the estimate, $\hat{x}_{.80}$, is computed from a sample of size $n$, there is a $100(1-\alpha) \%$ chance that the true value, $x_{.80}$, is between $\hat{x}_{.80}-C \sigma$ and $\hat{x}_{.80}+C \sigma$, where $C$ and $\alpha$ are to be specified. Table 4 gives the values of $n_{0}$ for various values of $C$ and $\alpha$ from which the required sample size $n$ can be computed from equation (4).

TABLE 4. Required $n_{0}$ Values for a $100(1-\alpha) \%$ Confidence Interval for $X_{.80}$ with Half-Width Co MBtu

C

$1 / 2$

$1 / 3$

$1 / 4$

$1 / 5$
$80 \%$

14

31

54

84
$90 \%$

22

50

89

138
$95 \%$

32

71

126

196

Using Hospitals as an example, Table 1 gives $K_{80}=144$ and $S_{76}=$ 109.22 which is used in place of $\sigma$. Table 4 shows that a $90 \%$ confidence interval for $X_{.80}$ with half-width $\sigma / 4 \doteq 109.22 / 4=27.31 \mathrm{MBtu}$ would require $n_{0}=89$, yielding a sample size $n=55$. Whereas Table 3 shows that a $90 \%$ confidence interval for $x_{.80}$ with half-width 5 MBtu would require a sample size $n=137$. Clearly, requiring a less precise estimate substantially reduces the necessary sample size.

Another important consideration in determining sample size is the hypothes is to be tested and the desired power or sensitivity of the test for detecting departures from the hypothesis. Specifically, an objective of this survey will be to test, for each building type, the null hypothes is that the population mean of $x$, say $\mu_{X}$, is equal to a specified value, say $\mu_{0}$, against the alternative hypothes is that $\mu_{x}$ differs from $\mu_{0}$. For example, it may be desirable to test the hypothesis that the mean $x$ value, ${ }_{x}$, for Large Office Buildings is $\mu_{0}=-40 \mathrm{MBtu}$, i.e., that in the population, Large 
Office Buildings have $D E C_{80}$ values which are an average of $40 \mathrm{MBtu}$ below the ir ${ }^{D E B} 80$ values. Clearly, if $\mu_{x}$ and $\mu_{0}$ differ "greatly" it is desirable to be able to detect this difference with a high probability. That is, when $\mu_{x}-\mu_{0}$ is "large", it is desirable to have a high probability of rejecting the null hypothesis in favor of the alternative. This probability is called the power of the test and depends on:

1. the size of $\mu_{x}-\mu_{0}$,

2. the significance level of the test, and

3. the standard deviation of the sample mean, $\sigma \sqrt{\left(K_{80}-n\right) / n K_{80}}$.

For a fixed significance level and given values of $\mu_{x}-\mu_{0}$ and $k_{80}$, the power of the test increases as $n$ increases. Thus, if $\sigma$ is known $\left(S_{76}\right.$ will be used as an approximation) it is possible to select a sample size, $n$, such that the power of the test is "sufficiently large" when $\mu_{x}-\mu_{0}$ exceeds a specified value.

The strategy for selecting a sample size for each building type will follow three steps.

1. Using equation 3 or 4 , find an "initial" sample size that satisfies the requirements for the desired precision of the estimate of the 80 th percentile.

2. Using the sample size from Step 1 and the desired significance level, compute the power of the test of the hypothesis that $\mu_{x}=\mu_{0}$ for various value of $\mu_{x}-\mu_{0}$.

3. If a more powerful test is desired, the sample size must be increased.

For the purpose of these comparisons, "power tables" are given in Appendix A. There are three tables for each building type which correspond to the significance levels $.20, .10$, and .05 . Each row of a table corresponds to a 
sample size $n$, and each column corresponds to a value of $\mu_{x}-\mu_{0}$. The body of the table gives the power of the test at each significance level/sample size/ $\mu_{x}-\mu_{0}$ combination.

Using Large Office Buildings as an example, Table A.I gives the power of the test when the significance level is .20 . Thus, if a sample of size $n=50$ is taken and the value of $\mu_{x}-\mu_{0}$ is 7.5MBtu, the power of the test is .9608. Figure 2 displays a plot of the power of the test v.s. $\mu_{x}-\mu_{0}$ (i.e., a power curve) for each of the sample sizes 25,50,100 and 200 . 


\section{SAMPLE SIZE RECOMMENDATIONS}

Using the information from the previous section, especially Tables 3 and 4 and Appendix A, it is possible to objectively determine a satisfactory sampling plan. However, keeping in mind the limited budget for the survey and the as yet unknown cost of analyzing a building, it is practical to consider several alternative sampling plans.

By way of review, the objectives of the survey for each building type are:

1. to construct a $100(1-\alpha) \%$ confidence interval for $x .80$ with a specified half-width, and

2. to test the hypothesis that $\mu_{x}=\mu_{0}$ with a specified level of significance and "sufficiently high" power when $\mu_{x}-\mu_{0}$ exceeds a specified value.

Tables 5, 6 and 7 give three sample sizes for each building type and the necessary information for evaluating and comparing "how we11" the objectives of the survey would be met if one of these sample sizes is used. For example, for Large Office Buildings, Table 5 indicates that a sample of size $n$ $=92$ is necessary to construct a $95 \%$ confidence interval for $X .80$ with halfwidth 5MBtu. For a sample of this size $(n=92)$ the power of the test of the hypothes is that $\mu_{x}=\mu_{0}$ is:

1. $80 \%$ when the significance level of the test is .20 and $\mu_{x}-\mu_{0}=3.8$,

2. $80 \%$ when the significance level is .10 and $\mu_{x}-\mu_{0}=4.4$,

3. $90 \%$ when the significance leve 1 is .20 and $\mu_{x}-\mu_{0}=4.6$, and

4. $90 \%$ when the significance leve 1 is .10 and $\mu_{x}-\mu_{0}=5.2$. 


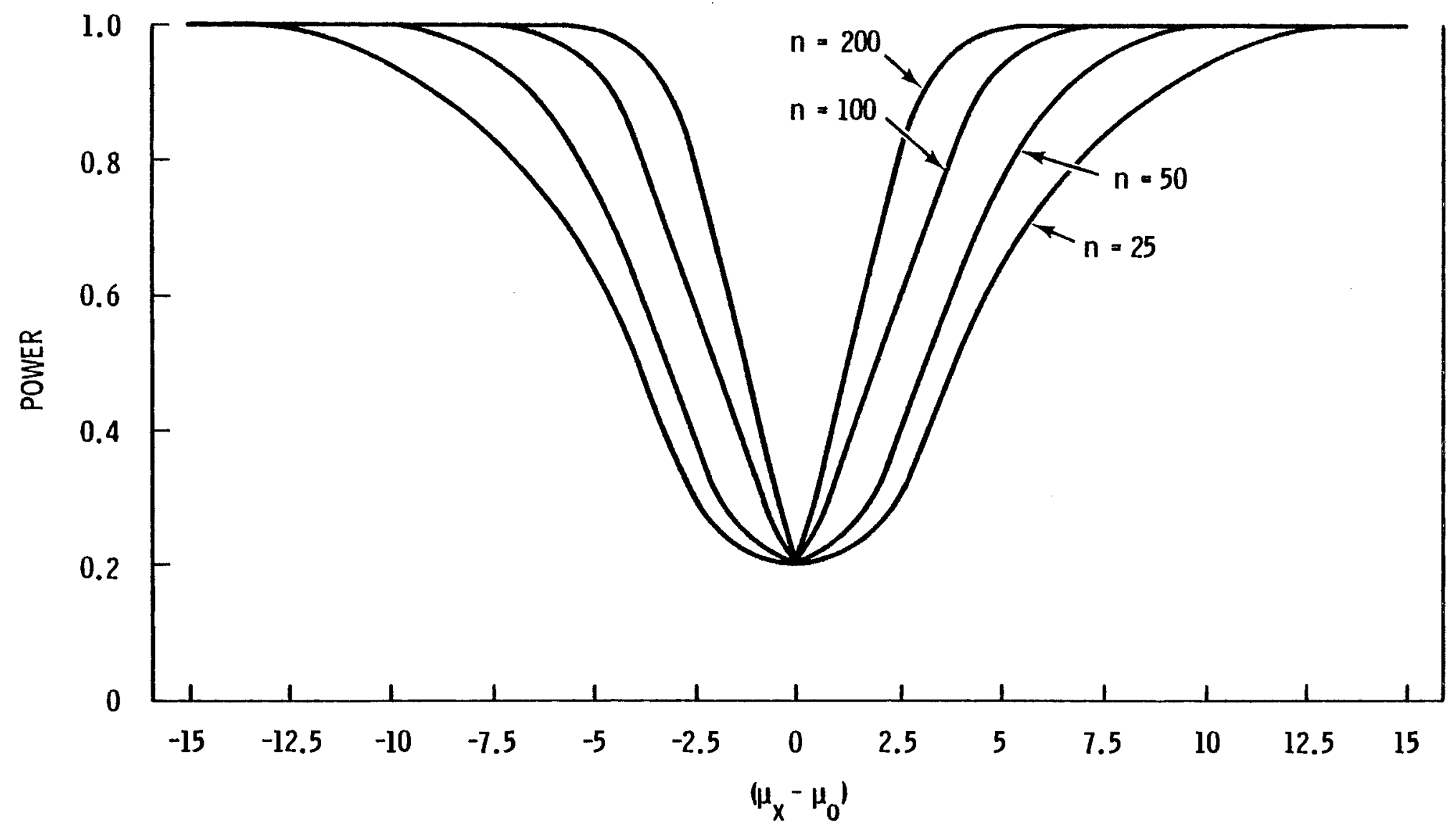

Figure 2. Power Curves for Large Office Buildings

When the Significance Level is . 20 . 
Tables 6 and 7 indicate that samples of size $n=67$ and $n=42$ would be required to construct $90 \%$ and $80 \%$ confidence intervals, respectively, for $X .80$ with half-widths 5MBtu. As expected, the power of the hypothesis test decreases as the sample size decreases.

The sample sizes given in Tables 5, 6 and 7 are computed using equations (3) and (4) by specifying a confidence level and a half-width for the confidence interval for $x_{.80}$. The scheme used to specify a half-width for a particular building type takes into account its relative importance to the study. Large Office, Small Office, Low-rise Apartments, High-rise Apartments, and Elementary School buildings have highest priority, and the corresponding half-widths are 5MBtu. Secondary Schools and Clinics have the next highest priority with half-widths of $(\sigma / 4)$ MBtu. Hospitals, Nursing Homes, Stores, and Shopping Centers are lower priority with half-widths of $(\sigma / 3)$ MBtu. The lowest priority building types are Warehouses, Hotel/Motel, Theater/Auditorium, Community Center, and Gymnasium with half-widths of $(\sigma / 2) M B t u$.

As indicated in the above example, the confidence level of the confidence interval for $X_{.80}$ is $95 \%$ for Table $5,90 \%$ for Table 6 , and $80 \%$ for Table 7 , while the half-widths are constant for all tables. This accounts for the decrease in the sample sizes from Table 5 to Table 7. The sampling plan represented by Table 5 with its larger sample sizes would be most likely to detect differences between $\mu_{x}$ and $\mu_{0}$ but it requires a total of 1141 buildings which may exceed the present budget.

The per building cost of data collection represents a significant unknown. It is recommended that data collection begin with the highest priority building type (Large Office Buildings) using the plan represented by Table 5 . An estimate of per building cost can be calculated after data collection has been completed for these buildings. From this estimate, some guidance may be obtained in determining the total number of buildings the budget will allow. At that time, a decision can be made to select a sampling plan for the remaining building types which best utilizes the remaining funds. However, every effort should be made to use the larger sample sizes for the higher priority building types to ensure sufficient precision and power. 
TABLE 5

\begin{tabular}{|c|c|c|c|c|c|c|}
\hline \multirow[t]{2}{*}{ Building Type } & \multirow{2}{*}{$\begin{array}{l}\text { Sample } \\
\text { Size } \\
\mathrm{n} \\
\end{array}$} & \multirow[t]{2}{*}{$\begin{array}{l}\text { Half-Width } \\
\text { of } 95 \% \text { CI. } \\
\text { for } X .80\end{array}$} & \multicolumn{4}{|c|}{$\begin{array}{l}\text { Value of }\left(\mu_{x}-\mu_{0}\right) \text { for which } \\
\text { the power of the test is } \\
80 \% \\
\end{array}$} \\
\hline & & & $.20 *$ & $.10 \star$ & $.20 \star$ & $.10 \star$ \\
\hline Large Office & 92 & 5 & 3.8 & 4.4 & 4.6 & 5.2 \\
\hline Sma11 Office & 199 & 5 & 3.8 & 4.4 & 4.6 & 5.2 \\
\hline Low-rise Apts. & 108 & 5 & 3.8 & 4.4 & 4.6 & 5.2 \\
\hline High-rise Apts. & 99 & 5 & 3.8 & 4.4 & 4.6 & 5.2 \\
\hline Elem School & 70 & 5 & 38 & 4.4 & 46 & 5.2 \\
\hline Sec. School & 104 & 7.2 & 5.5 & 6.4 & 6.6 & 7.5 \\
\hline Clinic & 118 & 10.1 & 7.6 & 8.9 & 9.2 & 10.5 \\
\hline Hospital & 48 & 36.4 & 27.5 & 32.0 & 33.0 & 37.7 \\
\hline Nurs. Home & 15 & 13.3 & 10.0 & 11.7 & 12.0 & 13.7 \\
\hline Store & 71 & 10.4 & 7.8 & 9.2 & 9.5 & 10.8 \\
\hline Shop. Center & 67 & 10.4 & 7.9 & 9.2 & 9.5 & 10.8 \\
\hline Warehouse & 32 & 9.8 & 7.4 & 8.6 & 8.9 & 10.1 \\
\hline Hote1/Mote1 & 31 & 8.7 & 6.5 & 7.6 & 7.9 & 9.0 \\
\hline Theater/Aud. & 28 & 11.6 & 8.7 & 10.2 & 10.5 & 12.0 \\
\hline Comm. Center & 28 & 11.1 & 8.3 & 96 & 9.9 & 11.3 \\
\hline Gymnasium & 31 & 17.8 & 13.3 & 15.5 & 16.0 & 18.3 \\
\hline
\end{tabular}

Total

1141

*Significance level of the test 
TABLE 6

\begin{tabular}{|c|c|c|c|c|c|c|}
\hline \multirow[t]{2}{*}{ Building Type } & \multirow{2}{*}{$\begin{array}{c}\text { Sample } \\
\text { Size } \\
n \\
\end{array}$} & \multirow[t]{2}{*}{$\begin{array}{l}\text { Half-Width } \\
\text { of } 90 \% \mathrm{CI} \text {. } \\
\text { for } X .80\end{array}$} & \multicolumn{4}{|c|}{$\begin{array}{c}\text { Value of }\left(\mu_{x}-\mu_{0}\right) \text { for which } \\
\text { the power of the test is } \\
80 \% \\
\end{array}$} \\
\hline & & & $.20 \star$ & $.10^{\star}$ & $.20 \star$ & $.10^{\star}$ \\
\hline Large Office & 67 & 5 & 4.5 & 5.3 & 5.4 & 6.2 \\
\hline Small Office & 140 & 5 & 4.5 & 5.3 & 5.4 & 6.2 \\
\hline Low-rise Apts. & 76 & 5 & 4.5 & 5.3 & 5.4 & 6.2 \\
\hline High-rise Apts. & 72 & 5 & 4.5 & 5.3 & 5.4 & 6.2 \\
\hline Elem. School & 52 & 5 & 4.5 & 5.3 & 5.4 & 6.2 \\
\hline Sec. School & 78 & 7.2 & 6.5 & 7.6 & 7.8 & 8.9 \\
\hline Crinic & 85 & 10.1 & 9.1 & 10.6 & 11.0 & 12.5 \\
\hline Hospital & 38 & 36.4 & 32.8 & 38.4 & 39.6 & 45.2 \\
\hline Nurs. Home & 14 & 13.3 & 11.9 & 13.9 & 14.3 & 16.4 \\
\hline Store & 50 & 10.4 & 9.3 & 10.9 & 11.3 & 12.9 \\
\hline Shop. Center & 49 & 10.4 & 9.4 & 10.9 & 11.3 & 12.9 \\
\hline Warehouse & 22 & 9.8 & 8.9 & 10.4 & 10.7 & 12.2 \\
\hline Hotel/Motel & 22 & 8.7 & 7.8 & 9.2 & 9.5 & 10.8 \\
\hline Theater/Aud. & 20 & 11.6 & 10.5 & 12.3 & 12.7 & 14.5 \\
\hline Comm. Center & 20 & 11.1 & 10.0 & 11.7 & 12.1 & 13.8 \\
\hline Gymnas i um & 22 & 17.8 & 16.0 & 18.7 & 19.3 & 22.0 \\
\hline
\end{tabular}

Total

827

*Significance level of the test 


\begin{tabular}{|c|c|c|c|c|c|c|}
\hline \multirow[t]{2}{*}{ Building Type } & \multirow{2}{*}{$\begin{array}{c}\text { Sample } \\
\text { Size } \\
\mathrm{n} \\
\end{array}$} & \multirow[t]{2}{*}{$\begin{array}{l}\text { Half-Width } \\
\text { of } 80 \% \mathrm{CI} \text {. } \\
\text { for } X .80\end{array}$} & \multicolumn{4}{|c|}{$\begin{array}{l}\text { Value of }\left(\mu_{x}-\mu_{0}\right) \text { for which } \\
\text { the power of the test is } \\
80 \% \\
\end{array}$} \\
\hline & & & $.20^{*}$ & $.10 \star$ & $.20 *$ & $.10 *$ \\
\hline Large Office & 42 & 5 & 5.7 & 6.7 & 6.9 & 7.9 \\
\hline Small Office & 85 & 5 & 5.7 & 6.7 & 6.9 & 7.9 \\
\hline Low-rise Apts. & 46 & 5 & 5.7 & 6.7 & 6.9 & 7.9 \\
\hline High-rise Apts. & 46 & 5 & 5.7 & 6.7 & 6.9 & 7.9 \\
\hline Elem. School & 34 & 5 & 5.7 & 6.7 & 6.9 & 7.9 \\
\hline Sec. School & 50 & 7.2 & 8.3 & 9.8 & 9.7 & 11.5 \\
\hline Clinic & 54 & 10.1 & 11.7 & 13.7 & 14.1 & 16.1 \\
\hline Hospital & 26 & 36.4 & 41.6 & 48.8 & 50.3 & 57.4 \\
\hline Nurs. Home & 12 & 13.3 & 15.1 & 17.6 & 18.2 & 20.8 \\
\hline Store & 31 & 10.4 & 11.9 & 13.9 & 14.3 & 16.4 \\
\hline Shop. Center & 31 & 10.4 & 11.9 & 13.9 & 14.3 & 16.4 \\
\hline Warehouse & 14 & 9.8 & 11.1 & 13.0 & 13.4 & 15.3 \\
\hline Hotel/Motel & 14 & 8.7 & 9.8 & 11.5 & 11.9 & 13.6 \\
\hline Theater/Aud. & 13 & 11.6 & 13.2 & 15.4 & 15.9 & 18.2 \\
\hline Cormm. Center & 14 & 11.1 & 12.6 & 14.7 & 15.2 & 17.3 \\
\hline Gymnasium & 14 & 17.8 & 20.0 & 23.5 & 24.2 & 27.6 \\
\hline
\end{tabular}

Total

526

*Significance level of the test 
The discussion, thus far, has dealt with determining how many buildings of each type should be included in the study. This section gives the details of the sampling scheme that will be implemented once the sample sizes have been determined. For notational simplicity and clarity of presentation, it is necessary to narrow the scope of the subsequent discussion to a single building type. This is consistent with previous sections, since a "separate survey" has been recormended for each building type.

The following definitions are helpful in subsequent discussions:

$k_{i}=$ the total number of buildings in the $i^{\text {th }}$ strata that are listed on the 1980 F.W. Dodge tapes.

$N_{i}=$ the total number of buildings in the $i^{\text {th }}$ strata that are listed on the $1980 \mathrm{~F} . \mathrm{W}$. Dodge tapes and that have construction plans available. The value of $N_{i}$ is unknown.

$L \quad=$ the number of strata for which $K_{j}>0$ and $N_{i}>0$.

$K=\sum_{i} K_{i}=$ the total number of buildings in the U.S.A. that are listed on the $1980 \mathrm{F.W}$. Dodge tapes. (This quantity was denoted $K_{80}$ in previous sections).

$N=\sum_{i} N_{i}=$ the total number of buildings in the U.S.A. that are listed on the $1980 \mathrm{~F} . \mathrm{W}$. Dodge tapes and that have construction plans available. The value of $N$ is unknown. 
$P_{i}=K_{i} / K$ expresses the total number of buildings in the $i^{\text {th }}$

strata as a percent of the total number of buildings in the

U.S.A.

$\mathrm{n} \quad=$ the proposed sample size.

$n_{i}=n_{i}$ rounded up to the next whole number. This will be the sample size for the $i^{\text {th }}$ strata, (i.e., the number of buildings from the $i^{\text {th }}$ strata that will be included in the survey). By rounding up to the next integer, $n_{i}$ will always be at least one unless $K_{i}$ is zero.

$n *=\sum_{i} n_{i}=$ the actual total sample size. $n *$ will usually be larger than $n$ due to rounding $n_{i}$.

For the particular building type being sampled, the sampling scheme will proceed as follows. A list of all buildings from the $1980 \mathrm{~F} . W$. Dodge tapes will be created on a computer file. Each building will be assigned a climate zone code (an integer between 1 and 7 ) and a Census Division code (an integer between 1 and 9) depending upon the location (state and county) of the building site, see Figure 1. It will then be possible to determine how many buildings from each of the 38 strata are listed on the $1980 \mathrm{~F} . W$. Dodge tapes. That is, the values of the $k_{i}$ 's can be determined.

For each of the strata with $k_{i}>0$, a distinct random integer between 1 and $k_{i}$ will be assigned to each of the $k_{i}$ buildings. The list of buildings will then be sorted so that the order of the buildings on the list is an ascending order of the random integers. Starting at the top of the sorted list, the first $n_{i}$ buildings will be included in the sample. If any of 
these $n_{i}$ buildings do not have construction plans available, the sampling continues by including the $\left(n_{i}+1\right)$ th, $\left(n_{j}+2\right)$ th, etc. buildings from the sorted list until there are $n_{i}$ buildings in the sample which do have construction plans available. Let $k_{i}$ denote the total number of buildings actually sampled in order to have $n_{i}$ buildings in the sample which have construction plans available.

One of the following situations may occur for some strata:

1. $K_{i}>0$ and $N_{i}<n_{j}$, or

2. $K_{i}>0$ and $N_{i}=0$.

If situation 1 occurs, then all of the $N_{i}$ buildings with construction plans available will be included in the sample. In this case, a re-definition of terms gives $k_{i}=k_{i}$ and $n_{i}=N_{i}$. Since the sample size for these strata will be smaller than planned, it may be desirable to reallocate the "unused portion" of the overall sample size to enlarge the sample sizes in other strata, or for other building types.

If situation 2 occurs, the particular strata will be dropped from the survey and the "unused portion" of the sample size may be reallocated as discussed above.

For the $i^{\text {th }}$ strata (except situation 2, described above) there will be $k_{i}$ buildings in the sample, of which $n_{j}$ have construction plans available. The $\left(k_{i}-n_{i}\right)$ buildings which do not have construction plans available will be treated as "non-respondents".

The construction plans for those buildings in the sample which have construction plans available will be analyzed by an architect who will complete a survey form for each building. The data from the survey forms will be used as input for the DOE2.1 computer program which will determine the $D C_{80}$ value for each building. 
The $D E B_{80}$ value for each building will be determined by the procedure outlined in the November 28, 1979 Federal Register, Vol. 44, No. 230, page 68180 .

The quantities $x_{i j}=\left(D E C_{80}-D^{D E B} 80\right)$ for $j^{\text {th }}$ building in the $i^{\text {th }}$ strata will be computed, and the estimation procedures described below will be used to construct confidence intervals for the national mean, $\mu x$, and the 80 th percentile, $X_{.} 80^{\text {. }}$

The population of buildings in strata $i$ can be viewed as two domains. The $N_{j}$ buildings with construction plans available are domain 1 , and the $\left(K_{i}-N_{i}\right)$ remaining buildings are domain 2 . Let $\mu_{1 j}$ and $\mu_{2 i}$ denote the population mean of $X$ for domain 1 and domain 2 of the $i^{\text {th }}$ strata, respectively. Since only the buildings from domain 1 can be analyzed, the sample mean of $X$ for the $i^{\text {th }}$ strata, $\bar{X}_{j}$, will be an unbiased estimate of $\mu_{1 i}$. The estimate of the overall population mean, $\mu_{x}$, will be

$$
\bar{x}=\frac{\sum_{i=1}^{L} k_{i} \bar{x}_{i}}{k}
$$

The expectation of $\bar{x}$ in (5) is

$$
E(\bar{X})=\frac{\sum_{i=1}^{L} K_{i} \mu_{i}}{K}+\frac{\sum_{i=1}^{K}\left(K_{i}-N_{i}\right)\left(\mu_{1 i}-\mu_{2 i}\right)}{K}
$$

where $\mu_{i}$ is the population mean of the $i^{\text {th }}$ strata, so that (6) can be rewritten as

$$
\left[E(\bar{X})-\mu_{X}\right]=\sum_{i=1}^{L} \frac{\left(K_{i}-N_{i}\right)\left(\mu_{1 i}-\mu_{2 i}\right)}{K},
$$


The term on the right side of equation (7) is the bias of the estimator $\bar{x}$ in (5). Thus, in order for $\bar{x}$ to estimate $\mu_{x}$ unbiasedly, either

$$
\begin{aligned}
& \text { 1. } K_{i}=N_{i} \text { must hold or } \\
& \text { 2. } \mu_{1 i}=\mu_{2 i} \text { must hold }
\end{aligned}
$$

for every strata.

It will be assumed for this study that the difference $\left(\mu_{1 j}-\mu_{2 j}\right)$ is negligible for every strata. In addition, it is projected that the ratio $N_{j} / K_{j}$ is "near one", say $95 \%$, so that $\left(K_{i}-N_{j}\right) / K$ in equation (7) is sma11, say $5 \%$. Thus, under these conditions, the bias of $\mathbb{Z}$ as an estimate of $\mu_{x}$ can be ignored without serious consequences.

To construct a confidence interval for $\mu_{x}$ it is necessary to estimate the variance of $\bar{x}$. It is convenient to consider first the variance of $x_{i j}$, which is given by

$$
V\left(x_{i j}\right)=\sigma^{2}\left(\frac{N-n^{\star}}{N}\right)
$$

It is assumed that the variance of $x_{i j}$ is constant for all strata. Thus, $\sigma^{2}$ can be estimated by the within strata mean square, given by

$$
s_{w}^{2}=\sum_{i=1}^{L} \sum_{j=1}^{n_{i}}\left(x_{i j}-\bar{x}_{i}\right)^{2} /\left(n^{*}-L\right),
$$

which has $(n *-L)$ degrees of freedom. The quantities $N_{j}$, and hence $N$ in equation (8), are unknown. However, it will be assumed that

$$
n_{i} / N_{i} \doteq k_{i} / k_{i}
$$


so that an estimate of $\mathrm{N}_{i}$ is given by

$$
\hat{N}_{i}=n_{i} K_{i} / k_{i}
$$

from which it is possible to estimate $N$ using the formula

$$
\hat{N}=\sum_{i=1}^{L} \hat{N}_{i}
$$

Substituting $S_{W}^{2}$ and $\hat{N}$ in place of $\sigma^{2}$ and $N$ in equation (8) yields an estimate of the variance of $x_{i j}$ which is given by

$$
s^{2}=s_{w}^{2}\left(\frac{\hat{N}-n^{*}}{\hat{N}}\right)
$$

Then the variance of $\bar{x}_{i}$ is estimated by

$$
\hat{v}\left(\bar{x}_{i}\right)=\frac{s^{2}}{n_{i}},
$$

and it follows that an estimate of the variance of $X$ is given by

$$
\hat{V}(\bar{x})=\frac{s^{2}}{k^{2}} \sum_{i=1}^{L} \frac{k_{i}{ }^{2}}{n_{i}}
$$

which has $\left(n^{\star}-L\right)$ degrees of freedom.

$$
\begin{gathered}
\text { A } 100(1-\alpha) \% \text { confidence interval for } \mu_{x} \text { is given by } \\
\quad \bar{X} \pm\left(t_{n *-L, \alpha / 2}\right) \sqrt{\hat{V}(\bar{X})}
\end{gathered}
$$


where $\bar{X}$ is given by equation $(5), \hat{V}(\bar{X})$ is given by equation (15) and where $t_{n^{*}-L}$ is the $\alpha / 2$ percentile of the Student's $t$ distribution with ( $n^{*}$ $L)$ degrees of freedom.

An estimate of the 80 th percentile, $x_{.80}$, is given by

$$
\hat{x}_{.80}=\bar{x}+\left(t_{n *-L}, .80\right) \sqrt{s^{2}}
$$

where $\bar{x}$ is given by equation (5), $s^{2}$ is given by equation (13), and where ${ }^{t} n^{*}-L, .80$ is the 80 th percentile of the Student's $t$ distribution with $\left(n^{\star}-L\right)$ degrees of freedom.

Using a propogation of error arguement, it can be shown that an estimate of the variance of $\hat{x}_{.80}$ is given by

$$
\hat{V}\left(\hat{X}_{.80}\right)=\hat{V}(\bar{X})+\left[\frac{s^{2} t^{2} n^{\star}-L, .80}{2\left(n^{*}-L\right)}\right],
$$

where $\hat{V}(\bar{X})$ is given by equation (15), $s^{2}$ is given by equation (13), and where $t_{n^{*}-L}, .80$ is the 80th percentile of the Student's $t$ distribution with $(n *-L)$ degrees of freedom.

A $100(1-\alpha) \%$ confidence interval for $x_{.80}$ is given by

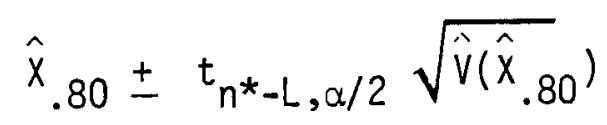

where $\hat{x}_{.80}$ is given by equation (17), $\hat{v}\left(\hat{x}_{.80}\right)$ is given by equation (18), and where $t_{n^{*}-L, \alpha / 2}$ is the $\alpha / 2$ percentile of the Student's $t$ distribution with $\left(n^{*}-L\right)$. degrees of freedom. 


\section{LIMITATIONS OF THE SAMPLE DESIGN}

A difficulty that will most likely be encountered, especially with the lower priority building types, stems from the fact that there are thirty-eight strata. When the proposed sample size is considerably smaller than the number of strata for which $k_{i}$ is larger than zero, it may be too costly to increase the sample size enough so that all strata are represented. There are several possible options in this case. Perhaps the most feasible for this study is the so-called "method of collapsed strata" discussed earlier, where several similar strata are combined to form one larger stratum. The number of strata is thus reduced, enabling the sampling scheme and data analys is described above to be implemented.

It must be emphasized that the proposed sample design is intended to yield sufficient information for making "satisfactory" inferences about nationwide compliance with $\mathrm{DEB}_{80}$ for the various building types. The intent is not to draw conclusions about compliance within individual climate zones or Census Divisions. If, for example, it is desirable to make inferences about compliance with $\mathrm{DEB}_{80}$ with in each Census Division, then sample sizes similar to those given in Tables 3 through 7 would be required for each Census Division. This would increase the total number of buildings required for the survey by as much as $900 \%$ or nine times the number required for nationwide inferences. Similarly, to make inferences about compliance with ${ }^{D E B}{ }_{80}$ with in each climate zone could require $700 \%$ or seven times the total sample size required for nationwide inferences. It can be seen by examination of Table 1 and Appendix $B$ that for some building types, $K_{80}$ is much smaller than $700 \%$ or $900 \%$ of the proposed sample size. Thus, for these particular building types, the above comments are over-pessimistic. However, the restricted budget for this project precludes taking large enough samples to make "satisfactory" inferences within individual climate zones or census divisions. 

APPENDIX A

POWER TABLES

A-1 
TABIE A. 1 LARGE OHFICE

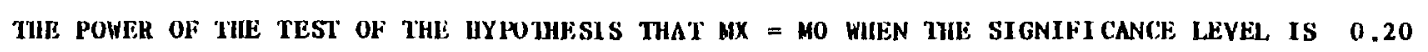

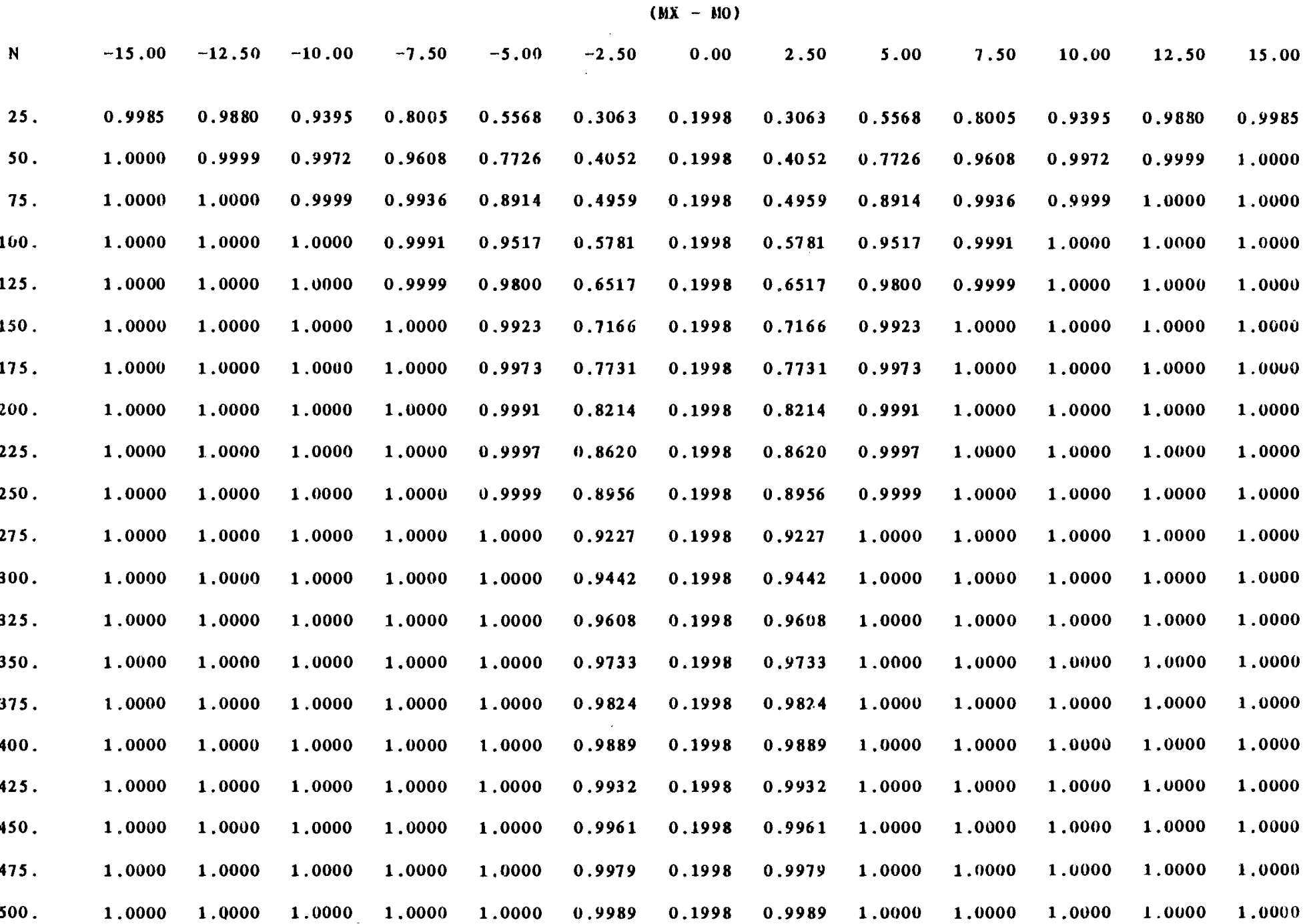


TAHLB A. 2 LARGE OFFICE

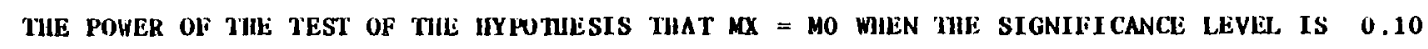

\begin{tabular}{|c|c|c|c|c|c|c|c|c|c|c|c|c|c|}
\hline & & & & & & & $M X-M 0)$ & & & & & & \\
\hline $\mathrm{N}$ & -15.00 & -12.50 & -10.00 & -7.50 & -5.00 & -2.50 & 0.00 & 2.50 & 5.00 & 7.50 & 10.00 & 12.50 & 15.00 \\
\hline 25 . & 0.9954 & 0.9710 & 0.8824 & 0.6842 & 0.4106 & 0.1837 & 0.1000 & 0.1837 & 0.4106 & 0.6842 & 0.8824 & 0.9710 & 0.9954 \\
\hline 50. & 1.0000 & 0.9997 & 0.9920 & 0.9188 & 0.6492 & 0.2679 & 0.1000 & 0.2679 & 0.6492 & 0.9188 & 0.9920 & 0.9997 & 1.0000 \\
\hline 75. & 1.0000 & 1.0000 & 0.9996 & 0.9833 & 0.8080 & 0.3512 & 0.1000 & 0.3512 & 0.8080 & 0.9833 & 0.9996 & 1.0000 & 1.0000 \\
\hline 100. & 1.0000 & 1.0000 & 1.0000 & 0.9972 & 0.9029 & 0.4321 & 0.1000 & 0.4321 & 0.9029 & 0.9972 & 1.0000 & 1.0000 & 1.0000 \\
\hline 125. & 1.0000 & 1.0000 & 1.0000 & 0.9996 & 0.9545 & 0.5095 & 0.1000 & 0.5095 & 0.9545 & 0.9996 & 1.0000 & 1.0000 & 1.0000 \\
\hline 150. & 1.0000 & 1.0000 & 1.0000 & 1.0000 & 0.9803 & 0.5824 & 0.1000 & 0.5824 & 0.9803 & 1.0000 & 1.0000 & 1.0000 & 1.0000 \\
\hline 175. & 1.0000 & 1.0000 & 1.0000 & 1.0000 & 0.9921 & 0.6498 & 0.1000 & 0.6498 & 0.9921 & 1.0000 & 1.0000 & 1.0000 & 1.0000 \\
\hline 200 . & 1.0000 & 1.0000 & 1.0000 & 1.0000 & 0.9971 & 0.7112 & 0.1000 & 0.7112 & 0.9971 & 1.0000 & 1.0000 & 1.0000 & 1.0000 \\
\hline 225. & 1.0000 & 1.0000 & 1.0000 & 1.0000 & 0.9990 & 0.7661 & 0.1000 & 0.7661 & 0.9990 & 1.0000 & 1.0000 & 1.0000 & 1.0000 \\
\hline 250 . & 1.0000 & 1.0000 & 1.0000 & 1.0000 & 0.9997 & 0.8142 & 0.1000 & 0.8142 & 0.9997 & 1.0000 & 1.0000 & 1.0000 & 1.0000 \\
\hline 275. & 1.0000 & 1.0000 & 1.0000 & 1.0000 & 0.9999 & 0.8555 & 0.1000 & 0.8555 & 0.9999 & 1.0000 & 1.0000 & 1.0000 & 1.0000 \\
\hline 300 . & 1.0000 & 1.0000 & 1.0000 & 1.0000 & 1.0000 & 0.8903 & 0.1000 & 0.8903 & 1.0000 & 1.0000 & 1.0000 & 1.0000 & 1.0000 \\
\hline 325. & 1.0000 & 1.0000 & 1.0000 & 1.0000 & 1.0000 & 0.9189 & 0.1000 & 0.9189 & 1.0000 & 1.0000 & 1.0000 & 1.0000 & 1.0000 \\
\hline 350. & 1.0000 & 1.0000 & 1.0000 & 1.0000 & 1.0000 & 0.9417 & 0.1000 & 0.9417 & 1.0000 & 1.0000 & 1.0000 & 1.0000 & 1.0000 \\
\hline 375. & 1.0000 & 1.0000 & 1.0000 & 1.0000 & 1.0000 & 0.9594 & 0.1000 & 0.9594 & 1.0000 & 1.0000 & 1.0000 & 1.0000 & 1.0000 \\
\hline 400. & 1.0000 & 1.0000 & 1.0000 & 1.0000 & 1.0000 & 0.9727 & 0.1000 & 0.9727 & 1.0000 & 1.0000 & 1.0000 & 1.0000 & 1.0000 \\
\hline 425. & 1.0000 & 1.0000 & 1.0000 & 1.0000 & 1.0000 & 0.9824 & 0.1000 & 0.9824 & 1.0000 & 1.0000 & 1.0000 & 1.0000 & 1.0000 \\
\hline 450 . & 1.0000 & 1.0000 & 1.0000 & 1.0000 & 1.0000 & 0.9892 & 0.1000 & 0.9892 & 1.0000 & 1.0000 & 1.0000 & 1.0000 & 1.0000 \\
\hline 475 . & 1.0000 & 1.0000 & 1.0000 & 1.0000 & 1.0000 & 0.9937 & 0.1000 & 0.9937 & 1.0000 & 1.0000 & 1.0000 & 1.0000 & 1.0000 \\
\hline 500. & 1.0000 & 1.0000 & 1.0000 & 1.0000 & 1.0000 & 0.9965 & 0.1000 & 0.9965 & 1.0000 & 1.0000 & 1.0000 & 1.0000 & 1.0000 \\
\hline
\end{tabular}


TABLE A. 3 LARGE OFFICE

THE POWFR OF THE TEST OF THE IIYHOHESIS THAT MX $=$ MO WHEN THE SIGNIFI CNNCI LEVEL IS 0.05

\begin{tabular}{|c|c|c|c|c|c|c|c|c|c|c|c|c|c|}
\hline \multirow[b]{2}{*}{$\mathbf{N}$} & \multicolumn{13}{|c|}{$(M X-M 0)$} \\
\hline & -15.00 & -12.50 & -10.00 & -7.50 & -5.00 & -2.50 & 0.00 & 2.50 & 5.00 & 7.50 & 10.00 & 12.50 & 15.00 \\
\hline 25 . & 0.9889 & 0.9430 & 0.8085 & 0.5652 & 0.2936 & 0.1091 & 0.0500 & 0.1091 & 0.2936 & 0.5652 & 0.8085 & 0.9430 & 0.9889 \\
\hline 50 . & 1.0000 & 0.9991 & 0.9819 & 0.8603 & 0.5271 & 0.1735 & 0.0500 & 0.1735 & 0.5271 & 0.8603 & 0.9819 & 0.9991 & 1.0000 \\
\hline 75. & 1.0000 & 1.0000 & 0.9989 & 0.9651 & 0.7108 & 0.2419 & 0.0500 & 0.2419 & 0.7108 & 0.9651 & 0.9989 & 1.0000 & 1.0000 \\
\hline 100. & 1.0000 & 1.0000 & 1.0000 & 0.9930 & 0.8372 & 0.3129 & 0.0500 & 0.3129 & 0.8372 & 0.9930 & 1.0000 & 1.0000 & 1.0000 \\
\hline 125. & 1.0000 & 1.0000 & 1.0000 & 0.9988 & 0.9155 & 0.3852 & 0.0500 & 0.3852 & 0.9155 & 0.9988 & 1.0000 & 1.0000 & 1.0000 \\
\hline 150. & 1.0000 & 1.0000 & 1.0000 & 0.9998 & 0.9595 & 0.4572 & 0,0500 & 0.4572 & 0.9595 & 0.9998 & 1.0000 & 1.0000 & 1.0000 \\
\hline 175. & 1.0000 & 1.0000 & 1.0000 & 1.0000 & 0.9821 & 0.5277 & 0.0500 & 0.5277 & 0.9821 & 1.0000 & 1.0000 & 1.0000 & 1.0000 \\
\hline 200. & 1.0000 & 1.0000 & 1.0000 & 1.0000 & 0.9927 & 0.5955 & 0.0500 & 0.5955 & 0.9927 & 1.0000 & 1.0000 & 1.0000 & 1.0000 \\
\hline 225. & 1.0000 & 1.0000 & 1.0000 & 1.0000 & 0.9973 & 0.6594 & 0.0500 & 0.6594 & 0.9973 & 1.0000 & 1.0000 & 1.0000 & 1.0000 \\
\hline 250. & 1.0000 & 1.0000 & 1.0000 & 1.0000 & 0.9991 & 0.7185 & 0.0500 & 0.7185 & 0.9991 & 1.0000 & 1.0000 & 1.0000 & 1.0000 \\
\hline 275. & 1.0000 & 1.0000 & 1.0000 & 1.0000 & 0.9997 & 0.7720 & 0.0500 & 0.7720 & 0.9997 & 1.0000 & 1.0000 & 1.0000 & 1.0000 \\
\hline 300. & 1.0000 & 1.0000 & 1.0000 & 1.0000 & 0.9999 & 0.8194 & 0.0500 & 0.8194 & 0.9999 & 1.0000 & 1.0000 & 1.0000 & 1.0000 \\
\hline 325. & 1.0000 & 1.0000 & 1.0000 & 1.0000 & 1.0000 & 0.8605 & 0.0500 & 0.8605 & 1.0000 & 1.0000 & 1.0000 & 1.0000 & 1.0000 \\
\hline 350 & 1.0000 & 1.0000 & 1.0000 & 1.0000 & 1.0000 & 0.8951 & 0.0500 & 0.8951 & 1.0000 & 1.0000 & 1.0000 & 1.0000 & 1.0000 \\
\hline 375. & 1.0000 & 1.0000 & 1.0000 & 1.0000 & 1.0000 & 0.9235 & 0.0500 & 0.9235 & 1.0000 & 1.0000 & 1.0000 & 1.0000 & 1.0000 \\
\hline 400. & 1.0000 & 1.0000 & 1.0000 & 1.0000 & 1.0000 & 0.9461 & 0.0500 & 0.9461 & 1.0000 & 1.0000 & 1.0000 & 1.0000 & 1.0000 \\
\hline 425. & 1.0000 & 1.0000 & 1.0000 & 1.0000 & 1.0000 & 0.9634 & 0.0500 & 0.9634 & 1.0000 & 1.0000 & 1.0000 & 1.0000 & 1.0000 \\
\hline 450. & 1.0000 & 1.0000 & 1.0000 & 1.0000 & 1.0000 & 0.9762 & 0.0500 & 0.9762 & 1.0000 & 1.0000 & 1.0000 & 1.0000 & 1.0000 \\
\hline 475. & 1.0000 & 1.0000 & 1.0000 & 1.0000 & 1.0000 & 0.9853 & 0.0500 & 0.9853 & 1.0000 & 1.0000 & 1.0000 & 1.0000 & 1.0000 \\
\hline 80 . & 1.0000 & 1.0000 & 1.0000 & 1.0000 & 1.0000 & 0.9914 & 0.0500 & 0.9914 & 1.0000 & 1.0000 & 1.0000 & 1.0000 & 1.0000 \\
\hline
\end{tabular}


TABLE A. 4 SMALI, OFFICE

TIE POWER OF THE TEST OF THE IYYOMIESIS THAT MX = MO MIEN THE SIGNIFI CANCE LEVH, IS 0.20

(KX - Mo)

\begin{tabular}{|c|c|c|c|c|c|c|c|c|c|c|c|c|c|}
\hline $\mathbf{N}$ & -15.00 & -12.50 & -10.00 & -7.50 & -5.00 & -2.50 & 0.00 & 2.50 & 5.00 & 7.50 & 10.00 & 12.50 & 15.00 \\
\hline 25. & 0.9556 & 0.8859 & 0.7608 & 0.5859 & 0.3983 & 0.2539 & 0.1998 & 0.2539 & 0.3983 & 0.5859 & 0.7608 & 0.8859 & 0.9556 \\
\hline 50. & 0.9984 & 0.9874 & 0.9376 & 0.7972 & 0.5539 & 0.3052 & 0.1998 & 0.3052 & 0.5539 & 0.7972 & 0.9376 & 0.9874 & 0.9984 \\
\hline 75. & 1.0000 & 0.9988 & 0.9851 & 0.9048 & 0.6732 & 0.3538 & 0.1998 & 0.3538 & 0.6732 & 0.9048 & 0.9851 & 0.9988 & 1.0000 \\
\hline 100. & 1.0000 & 0.9999 & 0.9967 & 0.9567 & 0.7633 & 0.3997 & 0.1998 & 0.3997 & 0.7633 & 0.9567 & 0.9967 & 0.9999 & 1.0000 \\
\hline 125. & 1.0000 & 1.0000 & 0.9993 & 0.9809 & 0.8301 & 0.4430 & 0.1998 & 0.4430 & 0.8301 & 0.9809 & 0.9993 & 1.0000 & 1.0000 \\
\hline 150. & 1.0000 & 1.0000 & 0.9999 & 0.9917 & 0.8792 & 0.4839 & 0.1998 & 0.4839 & 0.8792 & 0.9917 & 0.9999 & 1.0000 & 1.0000 \\
\hline 175. & 1.0000 & 1.0000 & 1.0000 & 0.9965 & 0.9148 & 0.5222 & 0.1998 & 0.5222 & 0.9148 & 0.9965 & 1.0000 & 1.0000 & 1.0000 \\
\hline 200 . & 1.0000 & 1.0000 & 1.0000 & 0.9985 & 0.9404 & 0.5583 & 0.1998 & 0.5583 & 0.9404 & 0.9985 & 1.0000 & 1.0000 & 1.6000 \\
\hline 25. & 1.0000 & 1.0000 & 1.0000 & 0.9994 & 0.9586 & 0.5920 & 0.1998 & 0.5920 & 0.9586 & 0.9994 & 1.0000 & 1.0000 & 1.0000 \\
\hline 250. & 1.0000 & 1.0000 & 1.0000 & 0.9998 & 0.9714 & 0.6237 & 0.1998 & 0.6237 & 0.9714 & 0.9998 & 1.0000 & 1.0000 & 1.0000 \\
\hline 75. & 1.0000 & 1.0000 & 1.0000 & 0.9999 & 0.9804 & 0.6532 & 0.1998 & 0.6532 & 0.9804 & 0.9999 & 1.0600 & 1.0000 & 1.0000 \\
\hline 300 & 1.0000 & 1.0000 & 1.0000 & 1.0000 & 0.9866 & 0.6808 & 0.1998 & 0.6808 & 0.9866 & 1.0000 & 1.0000 & 1.0000 & 1.0000 \\
\hline 325 . & 1.0000 & 1.0000 & 1.0000 & 1.0000 & 0.9909 & 0.7065 & 0.1998 & 0.7065 & 0.9909 & 1.0000 & 1.0000 & 1.0000 & 1.0000 \\
\hline 350. & 1.0000 & 1.0000 & 1.0000 & 1.0000 & 0.9939 & 0.7304 & 0.1998 & 0.7304 & 0.9939 & 1.0000 & 1.0000 & 1.0000 & 1.0000 \\
\hline 375 . & 1.0000 & 1.0000 & 1.0000 & 1.0000 & 0.9959 & 0.7526 & 0.1998 & 0.7526 & 0.9959 & 1.0000 & 1.0000 & 1.0000 & 1.0000 \\
\hline 400 . & 1.0000 & 1.0000 & 1.0000 & 1.0000 & 0.9973 & 0.7733 & 0.1998 & 0.7733 & 0.9973 & 1.0000 & 1.0000 & 1.0000 & 1.0000 \\
\hline 425. & 1.0000 & 1.0000 & 1.0000 & 1.0000 & 0.9982 & 0.7924 & 0.1998 & 0.7924 & 0.9982 & 1.0000 & 1.0000 & 1.0000 & 1.0000 \\
\hline 450 . & 1.0000 & 1.0000 & 1.0000 & 1.0000 & 0.9988 & 0.8101 & 0.1998 & 0.8101 & 0.9988 & 1.0000 & 1.0000 & 1.0000 & 1.0000 \\
\hline 75. & 1.0000 & 1.0000 & 1.0000 & 1.0000 & 0.9992 & 0.8265 & 0.1998 & 0.8265 & 0.9992 & 1.0000 & 1.0000 & 1.0000 & 1.0000 \\
\hline 00 . & 1.0000 & 1.0000 & 1.0000 & 1.0000 & 0.9995 & 0.8416 & 0.1998 & 0.8416 & 0.9995 & 1.0000 & 1.0000 & 1.0000 & 1.0000 \\
\hline
\end{tabular}


TABLE A. 5 SMALL OFFICE

TUE POWER OF THE TEST OF THE HYYOTHESIS THAT MX = MO WHLN THE SIGNIFICANCL LEVEL IS 0.10

\begin{tabular}{|c|c|c|c|c|c|c|c|c|c|c|c|c|c|}
\hline \multirow[b]{2}{*}{$\mathbf{N}$} & \multicolumn{13}{|c|}{$(M X-M 0)$} \\
\hline & -15.00 & -12.50 & -10.00 & -7.50 & -5.00 & -2.50 & 0.00 & 2.50 & 5.00 & 7.50 & 10.00 & 12.50 & 15.00 \\
\hline 25 . & 0.9097 & 0.8000 & 0.6348 & 0.4400 & 0.2619 & 0.1416 & 0.1000 & 0.1416 & 0.2619 & 0.4400 & 0.6348 & 0.8000 & 0.9097 \\
\hline 50. & 0.9951 & 0.9697 & 0.8794 & 0.6801 & 0.4076 & 0.1828 & 0.1000 & 0.1828 & 0.4076 & 0.6801 & 0.8794 & 0.9697 & 0.9951 \\
\hline 75. & 0.9998 & 0.9962 & 0.9648 & 0.8279 & 0.5332 & 0.2234 & 0.1000 & 0.2234 & 0.5332 & 0.8279 & 0.9648 & 0.9962 & 0.9998 \\
\hline 100. & 1.0000 & 0.9996 & 0.9906 & 0.9116 & 0.6378 & 0.2631 & 0.1000 & 0.2631 & 0.6378 & 0.9116 & 0.9906 & 0.9996 & 1.0000 \\
\hline 125. & 1.0000 & 1.0000 & 0.9976 & 0.9563 & 0.7228 & 0.3019 & 0.1000 & 0.3019 & 0.7228 & 0.9563 & 0.9976 & 1.0000 & 1.0000 \\
\hline 150. & 1.0000 & 1.0000 & 0.9994 & 0.9790 & 0.7904 & 0.3398 & 0.1000 & 0.3398 & 0.7904 & 0.9790 & 0.9994 & 1.0000 & 1.0000 \\
\hline 175. & 1.0000 & 1.0000 & 0.9999 & 0.9902 & 0.8432 & 0.3765 & 0.1000 & 0.3765 & 0.8432 & 0.9902 & 0.9999 & 1.0000 & 1.0000 \\
\hline 200 . & 1.0000 & 1.0000 & 1.0000 & 0.9955 & 0.8839 & 0.4120 & 0.1000 & 0.4120 & 0.8839 & 0.9955 & 1.0000 & 1.0000 & 1.0000 \\
\hline 225 & 1.0000 & 1.0000 & 1.0000 & 0.9980 & 0.9148 & 0.4463 & 0.1000 & 0.4463 & 0.9148 & 0.9980 & 1.0000 & 1.0000 & 1.0000 \\
\hline 250 . & 1.0000 & 1.0000 & 1.0000 & 0.9991 & 0.9380 & 0.4794 & 0.1000 & 0.4794 & 0.9380 & 0.9991 & 1.0000 & 1.0000 & 1.0000 \\
\hline 275 . & 1.0000 & 1.0000 & 1.0000 & 0.9996 & 0.9553 & 0.5111 & 0.1000 & 0.5111 & 0.9553 & 0.9996 & 1.0000 & 1.0000 & 1.0000 \\
\hline 300. & 1.0000 & 1.0000 & 1.0000 & 0.9998 & 0.9680 & 0.5416 & 0.1000 & 0.5416 & 0.9680 & 0.9998 & 1.0000 & 1.0000 & 1.0000 \\
\hline 325 & 1.0000 & 1.0000 & 1.0000 & 0.9999 & 0.9772 & 0.5707 & 0.1000 & 0.5707 & 0.9772 & 0.9999 & 1.0000 & 1.0000 & 1.0000 \\
\hline 350. & 1.0000 & 1.0000 & 1.0000 & 1.0000 & 0.9839 & 0.5984 & 0.1000 & 0.5984 & 0.9839 & 1.0000 & 1.0000 & 1.0000 & 1.0000 \\
\hline 375. & 1.0000 & 1.0000 & 1.0000 & 1.0000 & 0.9887 & 0.6249 & 0.1000 & 0.6249 & 0.9887 & 1.0000 & 1.0000 & 1.0000 & 1.0000 \\
\hline 400 . & 1.0000 & 1.0000 & 1.0000 & 1.0000 & 0.9921 & 0.6501 & 0.1000 & 0.6501 & 0.9921 & 1.0000 & 1.0000 & 1.0000 & 1.0000 \\
\hline 425. & 1.0000 & 1.0000 & 1.0000 & 1.0000 & 0.9946 & 0.6739 & 0.1000 & 0.6739 & 0.9946 & 1.0000 & 1.0000 & 1.0000 & 1.0000 \\
\hline 450 . & 1.0000 & 1.0000 & 1.0000 & 1.0000 & 0.9962 & 0.6966 & 0.1000 & 0.6966 & 0.9962 & 1.0000 & 1.0000 & 1.0000 & 1.0000 \\
\hline 5 . & 1.0000 & 1.0000 & 1.0000 & 1.0000 & 0.9974 & 0.7179 & 0.1000 & 0.7179 & 0.9974 & 1.0000 & 1.0000 & 1.0000 & 1.0000 \\
\hline 500. & 1.0000 & 1.0000 & 1.0000 & 1.0000 & 0.9982 & 0.7381 & 0.1000 & 0.7381 & 0.9982 & 1.0000 & 1.0000 & 1.0000 & 1.0000 \\
\hline
\end{tabular}


TABLE A. 6 SMALL OFFICE

THE POWER OF THE TESI OF THE HXPUDIESIS THAT MX = MO WIEN THE SIGNIFICANCE I.EVEL IS 0.05

\begin{tabular}{|c|c|c|c|c|c|c|c|c|c|c|c|c|c|}
\hline \multirow[b]{2}{*}{$N$} & \multicolumn{13}{|c|}{$(H X-M O)$} \\
\hline & -15.00 & -12.50 & -10.00 & -7.50 & -5.00 & -2.50 & 0.00 & 2.50 & 5.00 & 7.50 & 10.00 & 12.50 & 15.00 \\
\hline 25 . & 0.8470 & 0.7007 & 0.5117 & 0.3201 & 0.1687 & 0.0788 & 0.0500 & 0.0788 & 0.1687 & 0.3201 & 0.5117 & 0.7007 & 0.8470 \\
\hline 50 . & 0.9883 & 0.9407 & 0.8042 & 0.5607 & 0.2910 & 0.1085 & 0.0500 & 0.1085 & 0.2910 & 0.5607 & 0.8042 & 0.9407 & 0.9883 \\
\hline 75. & 0.9994 & 0.9908 & 0.9325 & 0.7359 & 0.4081 & 0.1388 & 0.0500 & 0.1388 & 0.4081 & 0.7359 & 0.9325 & 0.9908 & 0.9994 \\
\hline 100. & 1.0000 & 0.9988 & 0.9790 & 0.8498 & 0.5149 & 0.1697 & 0.0500 & 0.1697 & 0.5149 & 0.8498 & 0.9790 & 0.9988 & 1.0000 \\
\hline 125. & 1.0000 & 0.9999 & 0.9940 & 0.9183 & 0.6087 & 0.2009 & 0.0500 & 0.2009 & 0.6087 & 0.9183 & 0.9940 & 0.9999 & 1.0000 \\
\hline 150 . & 1.0000 & 1.0000 & 0.9984 & 0.9572 & 0.6889 & 0.2323 & 0.0500 & 0.2323 & 0.6889 & 0.9572 & 0.9984 & 1.0000 & 1.0000 \\
\hline 175. & 1.0000 & 1.0000 & 0.9996 & 0.9783 & 0.7558 & 0.2637 & 0.0500 & 0.2637 & 0.7558 & 0.9783 & 0.9996 & 1.0000 & 1.0000 \\
\hline 200 . & 1.0000 & 1.0000 & 0.9999 & 0.9893 & 0.8105 & 0.2949 & 0.0500 & 0.2949 & 0.8105 & 0.9893 & 0.9999 & 1.0000 & 1.0000 \\
\hline 225. & 1.0000 & 1.0000 & 1.0000 & 0.9948 & 0.8545 & 0.3259 & 0.0500 & 0.3259 & 0.8545 & 0.9948 & 1.0000 & 1.0000 & 1.0000 \\
\hline 250. & 1.0000 & 1.0000 & 1.0000 & 0.9976 & 0.8894 & 0.3565 & 0.0500 & 0.3565 & 0.8894 & 0.9976 & 1.0000 & 1.0000 & 1.0000 \\
\hline 275. & 1.0000 & 1.0000 & 1.0000 & 0.9989 & 0.9167 & 0.3867 & 0.0500 & 0.3867 & 0.9167 & 0.9989 & 1.0000 & 1.0000 & 1.0000 \\
\hline 300 . & 1.0000 & 1.0000 & 1.0000 & 0.9995 & 0.9378 & 0.4163 & 0.0500 & 0.4163 & 0.9378 & 0.9995 & 1.0000 & 1.0000 & 1.0000 \\
\hline 325 . & 1.0000 & 1.0000 & 1.0000 & 0.9998 & 0.9540 & 0.4453 & 0.0500 & 0.4453 & 0.9540 & 0.9998 & 1.0000 & 1.0000 & 1.0000 \\
\hline 350. & 1.0000 & 1.0000 & 1.0000 & 0.9999 & 0.9662 & 0.4737 & 0.0500 & 0.4737 & 0.9662 & 0.9999 & 1.0000 & 1.0000 & 1.0000 \\
\hline 375. & 1.0000 & 1.0000 & 1.0000 & 1.0000 & 0.9753 & 0.5012 & 0.0500 & 0.5012 & 0.9753 & 1.0000 & 1.0000 & 1.0000 & 1.0000 \\
\hline 400. & 1.0000 & 1.0000 & 1.0000 & 1.0000 & 0.9821 & 0.5280 & 0.0500 & 0.5280 & 0.9821 & 1.0000 & 1.0000 & 1.0000 & 1.0000 \\
\hline 425. & 1.0000 & 1.0000 & 1.0000 & 1.0000 & 0.9872 & 0.5539 & 0.0500 & 0.5539 & 0.9872 & 1.0000 & 1.0000 & 1.0000 & 1.0000 \\
\hline 450. & 1.0000 & 1.0000 & 1.0000 & 1.0000 & 0.9908 & 0.5790 & 0.0500 & 0.5790 & 0.9908 & 1.0000 & 1.0000 & 1.0000 & 1.0000 \\
\hline 475. & 1.0000 & 1.0000 & 1.0000 & 1.0000 & 0.9935 & 0.6032 & 0.0500 & 0.6032 & 0.9935 & 1.0000 & 1.0000 & 1.0000 & 1.0000 \\
\hline 500 . & 1.0000 & 1.0000 & 1.0000 & 1.0000 & 0.9954 & 0.6265 & 0.0500 & 0.6265 & 0.9954 & 1.0000 & 1.0000 & 1.0000 & 1.0000 \\
\hline
\end{tabular}


TABLE A. 7 LOW-RISE APARTBilin'S

THE POWER OF THE TEST OH THE HYPUMESIS THAT MX = MO WHEN THE SIGNIFICANCE LEVEL $1 \mathrm{~S}$ 0.20

\begin{tabular}{|c|c|c|c|c|c|c|c|c|c|c|c|c|c|}
\hline \multirow[b]{2}{*}{$\mathbf{N}$} & \multicolumn{13}{|c|}{$(M X-M O)$} \\
\hline & -15.00 & -12.50 & -10.00 & -7.50 & -5.00 & -2.50 & 0.00 & 2.50 & 5.00 & 7.50 & 10.00 & 12.50 & 15.00 \\
\hline 25. & 0.9972 & 0.9817 & 0.9216 & 0.7713 & 0.5310 & 0.2970 & 0.1998 & 0.2970 & 0.5310 & 0.7713 & 0.9216 & 0.9817 & 0.9972 \\
\hline 50 . & 1.0000 & 0.9998 & 0.9944 & 0.9432 & 0.7356 & 0.3844 & 0.1998 & 0.3844 & 0.7356 & 0.9432 & 0.9944 & 0.9998 & 1.0000 \\
\hline 75. & 1.0000 & 1.0000 & 0.9997 & 0.9871 & 0.8553 & 0.4627 & 0.1998 & 0.4627 & 0.8553 & 0.9871 & 0.9997 & 1.0000 & 1.0000 \\
\hline 100. & 1.0000 & 1.0000 & 1.0000 & 0.9973 & 0.9228 & 0.5324 & 0.1998 & 0.5324 & 0.9228 & 0.9973 & 1.0000 & 1.0000 & 1.0000 \\
\hline 125. & 1.0000 & 1.0000 & 1.0000 & 0.9994 & 0.9596 & 0.5943 & 0.1998 & 0.5943 & 0.9596 & 0.9994 & 1.0000 & 1.0000 & 1.0000 \\
\hline 150 . & 1.0000 & 1.0000 & 1.0000 & 0.9999 & 0.9792 & 0.6489 & 0.1998 & 0.6489 & 0.9792 & 0.9999 & 1.0000 & 1.0000 & 1.0000 \\
\hline 175. & 1.0000 & 1.0000 & 1.0000 & 1.0000 & 0.9895 & 0.6970 & 0.1998 & 0.6970 & 0.9895 & 1.0000 & 1.0000 & 1.0000 & 1.0000 \\
\hline 200 . & 1.0000 & 1.0000 & 1.0000 & 1.0000 & 0.9948 & 0.7391 & 0.1998 & 0.7391 & 0.9948 & 1.0000 & 1.0000 & 1.0000 & 1.0000 \\
\hline 225 . & 1.0000 & 1.0000 & 1.0000 & 1.0000 & 0.9974 & 0.7759 & 0.1998 & 0.7759 & 0.9974 & 1.0000 & 1.0000 & 1.0000 & 1.0000 \\
\hline 250 . & 1.0000 & 1.0000 & 1.0000 & 1.0000 & 0.9987 & 0.8080 & 0.1998 & 0.8080 & 0.9987 & 1.0000 & 1.0000 & 1.0000 & 1.0000 \\
\hline 275. & 1.0000 & 1.0000 & 1.0000 & 1.0000 & 0.9994 & 0.8358 & 0.1998 & 0.8358 & 0.9994 & 1.0000 & 1.0000 & 1.0000 & 1.0000 \\
\hline 300. & 1.0000 & 1.0000 & 1.0000 & 1.0000 & 0.9997 & 0.8599 & 0.1998 & 0.8599 & 0.9997 & 1.0000 & 1.0000 & 1.0000 & 1.0000 \\
\hline 325 . & 1.0000 & 1.0000 & 1.0000 & 1.0000 & 0.9999 & 0.8807 & 0.1998 & 0.8807 & 0.9999 & 1.0000 & 1.0000 & 1.0000 & 1.0000 \\
\hline 350 . & 1.0000 & 1.0000 & 1.0000 & 1.0000 & 0.9999 & 0.8986 & 0.1998 & 0.8986 & 0.9999 & 1.0000 & 1.0000 & 1.0000 & 1.0000 \\
\hline 375. & 1.0000 & 1.0000 & 1.0000 & 1.0000 & 1.0000 & 0.9140 & 0.1998 & 0.9140 & 1.0000 & 1.0000 & 1.0000 & 1.0000 & 1.0000 \\
\hline 400 . & 1.0000 & 1.0000 & 1.0000 & 1.0000 & 1.0000 & 0.9272 & 0.1998 & 0.9272 & 1.0000 & 1.0000 & 1.0000 & 1.0000 & 1.0000 \\
\hline 425. & 1.0000 & 1.0000 & 1.0000 & 1.0000 & 1.0000 & 0.9385 & 0.1998 & 0.9385 & 1.0000 & 1.0000 & 1.0000 & 1.0000 & 1.0000 \\
\hline 450 . & 1.0000 & 1.0000 & 1.0000 & 1.0000 & 1.0000 & 0.9481 & 0.1998 & 0.9481 & 1.0000 & 1.0000 & 1.0000 & 1.0000 & 1.0000 \\
\hline 475 . & 1.0000 & 1.0000 & 1.0000 & 1.0000 & 1.0000 & 0.9563 & 0.1998 & 0.9563 & 1.0000 & 1.0000 & 1.0000 & 1.0000 & 1.0000 \\
\hline 500. & 1.0000 & 1.0000 & 1.0000 & 1.0000 & 1.0000 & 0.9633 & 0.1998 & 0.9633 & 1.0000 & 1.0000 & 1.0000 & 1.0000 & 1.0000 \\
\hline
\end{tabular}




\section{TABLE A. 8 LOW-RISE APARTMENTS}

THE POWER OF TUE TEST OF THE HYPOHLSIS TILT MX = MO HIEN THE SIGNIFICANCE LEVE, IS 0.10

\begin{tabular}{|c|c|c|c|c|c|c|c|c|c|c|c|c|c|}
\hline $\mathbf{N}$ & -15.00 & -12.50 & -10.00 & -7.50 & -5.00 & -2.50 & 0.00 & 2.50 & 5.00 & 7.50 & 10.00 & 12.50 & 15.00 \\
\hline 25 . & 0.9918 & 0.9580 & 0.8538 & 0.6476 & 0.3850 & 0.1761 & 0.1000 & 0.1761 & 0.3850 & 0.6476 & 0.8538 & 0.9580 & 0.9918 \\
\hline 50. & 1.0000 & 0.9991 & 0.9852 & 0.8886 & 0.6045 & 0.2497 & 0.1000 & 0.2497 & 0.6045 & 0.8886 & 0.9852 & 0.9991 & 1.0000 \\
\hline 75 . & 1.0000 & 1.0000 & 0.9988 & 0.9690 & 0.7568 & 0.3200 & 0.1000 & 0.3200 & 0.7568 & 0.9690 & 0.9988 & 1.0000 & 1.0000 \\
\hline 100. & 1.0000 & 1.0000 & 0.9999 & 0.9921 & 0.8556 & 0.3864 & 0.1000 & 0.3864 & 0.8556 & 0.9921 & 0.9999 & 1.0000 & 1.0000 \\
\hline 125 . & 1.0000 & 1.0000 & 1.0000 & 0.9981 & 0.9167 & 0.4487 & 0.1000 & 0.4487 & 0.9167 & 0.9981 & 1.0000 & 1.0000 & 1.0000 \\
\hline 150 . & 1.0000 & 1.0000 & 1.0000 & 0.9996 & 0.9530 & 0.5065 & 0.1000 & 0.5065 & 0.9530 & 0.9996 & 1.0000 & 1.0000 & 1.0000 \\
\hline 175. & 1.0000 & 1.0000 & 1.0000 & 0.9999 & 0.9741 & 0.5598 & 0,1000 & 0.5598 & 0.9741 & 0.9999 & 1.0000 & 1.0000 & 1.0000 \\
\hline 200 . & 1.0000 & 1.0000 & 1.0000 & 1.0000 & 0.9860 & 0.6087 & 0.1000 & 0.6087 & 0.9860 & 1.0000 & 1.0000 & 1.0000 & 1.0000 \\
\hline 225 . & 1.0000 & 1.0000 & 1.0000 & 1.0000 & 0.9925 & 0.6533 & 0.1000 & 0.6533 & 0.9925 & 1.0000 & 1.0000 & 1.0000 & 1.0000 \\
\hline 250 . & 1.0000 & 1.0000 & 1.0000 & 1.0000 & 0.9961 & 0.6938 & 0.1000 & 0.6938 & 0.9961 & 1.0000 & 1.0000 & 1.0000 & 1.0000 \\
\hline 275 . & 1.0000 & 1.0000 & 1.0000 & 1.0000 & 0.9980 & 0.7303 & 0.1000 & 0.7303 & 0.9980 & 1.0000 & 1.0000 & 1.0000 & 1.0000 \\
\hline 300 & 1.0000 & 1.0000 & 1.0000 & 1.0000 & 0.9990 & 0.7631 & 0.1000 & 0.7631 & 0.9990 & 1.0000 & 1.0000 & 1.0000 & 1.0000 \\
\hline 325 . & 1.0000 & 1.0000 & 1.0000 & 1.0000 & 0.9995 & 0.7925 & 0.1000 & 0.7925 & 0.9995 & 1.0000 & 1.0000 & 1.0000 & 1.0000 \\
\hline 350 . & 1.0000 & 1.0000 & 1.0000 & 1.0000 & 0.9997 & 0.8187 & 0.1000 & 0.8187 & 0.9997 & 1.0000 & 1.0000 & 1.0000 & 1.0000 \\
\hline 375 . & 1.0000 & 1.0000 & 1.0000 & 1.0000 & 0.9999 & 0.8420 & 0.1000 & 0.8420 & 0.9999 & 1.0000 & 1.0000 & 1.0000 & 1.0000 \\
\hline 400 . & 1.0000 & 1.0000 & 1.0000 & 1.0000 & 0.9999 & 0.8626 & 0.1000 & 0.8626 & 0.9999 & 1.0000 & 1.0000 & 1.0000 & 1.0000 \\
\hline 425. & 1.0000 & 1.0000 & 1.0000 & 1.0000 & 1.0000 & 0.8808 & 0.1000 & 0.8808 & 1.0000 & 1.0000 & 1.0000 & 1.0000 & 1.0000 \\
\hline 450 . & 1.0000 & 1.0000 & 1.0000 & 1.0000 & 1.0000 & 0.8968 & 0.1000 & 0.8968 & 1.0000 & 1.0000 & 1.0000 & 1.0000 & 1.0000 \\
\hline 475 . & 1.0000 & 1.0000 & 1.0000 & 1.0000 & 1.0000 & 0.9109 & 0.1000 & 0.9109 & 1.0000 & 1.0000 & 1.0000 & 1.0000 & 1.0000 \\
\hline 500 & 1.0000 & 1.0000 & 1.0000 & 1.0000 & 1.0000 & 0.9232 & 0.1000 & 0.9232 & 1.0000 & 1.0000 & 1.0000 & 1.0000 & 1.0000 \\
\hline
\end{tabular}


TABLE A. 9 LOW-RISE APARIMENTS

TIE POWER OF THE TEST OF TUE HYPOHLSIS THAT HX $=$ MO WHEN THE SIGNIFICANCE LEVE, IS 0.05

\begin{tabular}{|c|c|c|c|c|c|c|c|c|c|c|c|c|c|}
\hline \multirow[b]{2}{*}{$\mathbf{N}$} & \multicolumn{13}{|c|}{$(M X-M 0)$} \\
\hline & -15.00 & -12.50 & -10.00 & -7.50 & -5.00 & -2.50 & 0.00 & 2.50 & 5.00 & 7.50 & 10.00 & 12.50 & 15.00 \\
\hline 25 . & 0.9816 & 0.9211 & 0.7697 & 0.5253 & 0.2711 & 0.1035 & 0.0500 & 0.1035 & 0.2711 & 0.5253 & 0.7697 & 0.9211 & 0.9816 \\
\hline 50 . & 0.9999 & 0.9976 & 0.9685 & 0.8171 & 0.4799 & 0.1592 & 0.0500 & 0.1592 & 0.4799 & 0.8171 & 0.9685 & 0.9976 & 0.9999 \\
\hline 75 . & 1.0000 & 1.0000 & 0.9968 & 0.9396 & 0.6484 & 0.2158 & 0.0500 & 0.2158 & 0.6484 & 0.9396 & 0.9968 & 1.0000 & 1.0000 \\
\hline 100. & 1.0000 & 1.0000 & 0.9997 & 0.9821 & 0.7721 & 0.2723 & 0.0500 & 0.2723 & 0.7721 & 0.9821 & 0.9997 & 1.0000 & 1.0000 \\
\hline 125. & 1. .0000 & 1.0000 & 1.0000 & 0.9951 & 0.8572 & 0.3280 & 0.0500 & 0.3280 & 0.8572 & 0.9951 & 1.0000 & 1.0000 & 1.0000 \\
\hline 150. & 1.0000 & 1.0000 & 1.0000 & 0.9987 & 0.9131 & 0.3822 & 0.0500 & 0.3822 & 0.9131 & 0.9987 & 1.0000 & 1.0000 & 1.0000 \\
\hline 175. & 1.0000 & 1.0000 & 1.0000 & 0.9997 & 0.9484 & 0.4345 & 0.0500 & 0.1345 & 0.9484 & 0.9997 & 1.0000 & 1.0000 & 1.0000 \\
\hline 200. & 1.0000 & 1.0000 & 1.0000 & 0.9999 & 0.9700 & 0.4843 & 0.0500 & 0.4843 & 0.9700 & 0.9999 & 1.0000 & 1.0000 & 1.0000 \\
\hline 225. & 1.0000 & 1.0000 & 1.0000 & 1.0000 & 0.9829 & 0.5315 & 0.0500 & 0.5315 & 0.9829 & 1.0000 & 1.0000 & 1.0000 & 1.0000 \\
\hline 250. & 1.0000 & 1.0000 & 1.0000 & 1.0000 & 0.9904 & 0.5759 & 0.0500 & 0.5759 & 0.9904 & 1.0000 & 1.0000 & 1.0000 & 1.0000 \\
\hline 275. & 1.0000 & 1.0000 & 1.0000 & 1.0000 & 0.9947 & 0.6173 & 0.0500 & 0.6173 & 0.9947 & 1.0000 & 1.0000 & 1.0000 & 1.0000 \\
\hline 300. & 1.0000 & 1.0000 & 1.0000 & 1.0000 & 0.9971 & 0.6559 & 0.0500 & 0.6559 & 0.9971 & 1.0000 & 1.0000 & 1.0000 & 1.0000 \\
\hline 325. & 1.0000 & 1.0000 & 1.0000 & 1.0000 & 0.9985 & 0.6915 & 0.0500 & 0.6915 & 0.9985 & 1.0000 & 1.0000 & 1.0000 & 1.0000 \\
\hline 350. & 1.0000 & 1.0000 & 1.0000 & 1.0000 & 0.9992 & 0.7242 & 0.0500 & 0.7242 & 0.9992 & 1.0000 & 1.0000 & 1.0000 & 1.0000 \\
\hline 375. & 1.0000 & 1.0000 & 1.0000 & 1.0000 & 0.9996 & 0.7541 & 0.0500 & 0.7541 & 0.9996 & 1.0000 & 1.0000 & 1.0000 & 1.0000 \\
\hline 400 & 1.0000 & 1.0000 & 1.0000 & 1.0000 & 0.9998 & 0.7814 & 0.0500 & 0.7814 & 0.9998 & 1.0000 & 1.0000 & 1.0000 & 1.0000 \\
\hline 425. & 1.0000 & 1.0000 & 1.0000 & 1.0000 & 0.9999 & 0.8062 & 0.0500 & 0.8062 & 0.9999 & 1.0000 & 1.0000 & 1.0000 & 1.0000 \\
\hline 450. & 1.0000 & 1.0000 & 1.0000 & 1.0000 & 0.9999 & 0.8286 & 0.0500 & 0.8286 & 0.9999 & 1.0000 & 1.0000 & 1.0000 & 1.0000 \\
\hline 475 . & 1.0000 & 1.0000 & 1.0000 & 1.0000 & 1.0000 & 0.8488 & 0.0500 & 0.8488 & 1.0000 & 1.0000 & 1.0000 & 1.0000 & 1.0000 \\
\hline 500 . & 1.0000 & 1.0000 & 1.0000 & 1.0000 & 1.0000 & 0.8669 & 0.0500 & 0.8669 & 1.0000 & 1.0000 & 1.0000 & 1.0000 & 1.0000 \\
\hline
\end{tabular}


TARLE A.10 H1GH-RISE AYARTMENTS

THE POWER OP TIE TEST OF THE HY YOMIESIS THAT MX $=$ MO WIEN MUE SIGNIFICANCE LEVEL IS 0.20

\begin{tabular}{|c|c|c|c|c|c|c|c|c|c|c|c|c|c|}
\hline \multirow[b]{2}{*}{$\mathbf{N}$} & \multicolumn{13}{|c|}{$(M X-M 0)$} \\
\hline & -15.00 & -12.50 & -10.00 & -7.50 & -5.00 & -2.50 & 0.00 & 2.50 & 5.00 & 7.50 & 10.00 & 12.50 & 15.00 \\
\hline 25. & 0.9975 & 0.9832 & 0.9257 & 0.7776 & 0.5364 & 0.2989 & 0.1998 & 0.2989 & 0.5364 & 0.7776 & 0.9257 & 0.9832 & 0.9975 \\
\hline 50 & 1.0000 & 0.9998 & 0.9956 & 0.9498 & 0.7486 & 0.3915 & 0.1998 & 0.3915 & 0.7486 & 0.9498 & 0.9956 & 0.9998 & 1.0000 \\
\hline 75. & 1.0000 & 1.0000 & 0.9998 & 0.9904 & 0.8719 & 0.4771 & 0.1998 & 0.4771 & 0.8719 & 0.9904 & 0.9998 & 1.0000 & 1.0000 \\
\hline 100 & 1.0000 & 1.0000 & 1.0000 & 0.9984 & 0.9386 & 0.5555 & 0.1998 & 0.5555 & 0.9386 & 0.9984 & 1.0000 & 1.0000 & 1.0000 \\
\hline 125. & 1.0000 & 1.0000 & 1.0000 & 0.9998 & 0.9723 & 0.6264 & 0.1998 & 0.6264 & 0.9723 & 0.9998 & 1.0000 & 1.0000 & 1.0000 \\
\hline 150 & 1.0000 & 1.0000 & 1.0000 & 1.0000 & 0.9883 & 0.6899 & 0.1998 & 0.6899 & 0.9883 & 1.0000 & 1.0000 & 1.0000 & 1.0000 \\
\hline 175. & 1.0000 & 1.0000 & 1.0000 & 1.0000 & 0.9954 & 0.7459 & 0.1998 & 0.7459 & 0.9954 & 1.0000 & 1.0000 & 1.0000 & 1.0000 \\
\hline 200 & 1.0000 & 1.0000 & 1.0000 & 1.0000 & 0.9983 & 0.7948 & 0.1998 & 0.7948 & 0.9983 & 1.0000 & 1.0000 & 1.0000 & 1.0000 \\
\hline 225. & 1.0000 & 1.0000 & 1.0000 & 1.0000 & 0.9994 & 0.8368 & 0.1998 & 0.8368 & 0.9994 & 1.0000 & 1.0000 & 1.0000 & 1.0000 \\
\hline 250. & 1.0000 & 1.0000 & 1.0000 & 1.0000 & 0.9998 & 0.8724 & 0.1998 & 0.8724 & 0.9998 & 1.0000 & 1.0000 & 1.0000 & 1.0000 \\
\hline 275. & 1.0000 & 1.0000 & 1.0000 & 1.0000 & 0.9999 & 0.9020 & 0.1998 & 0.9020 & 0.99 .99 & 1.0000 & 1.0000 & 1.0000 & 1.0000 \\
\hline 300 & 1.0000 & 1.0000 & 1.0000 & 1.0000 & 1.0000 & 0.9263 & 0.1998 & 0.9263 & 1.0000 & 1.0000 & 1.0000 & 1.0000 & 1.0000 \\
\hline 325 . & 1.0000 & 1.0000 & 1.0000 & 1.0000 & 1.0000 & 0.9457 & 0.1998 & 0.9457 & 1.0000 & 1.0000 & 1.0000 & 1.0000 & 1.0000 \\
\hline 350. & 1.0000 & 1.0000 & 1.0000 & 1.0000 & 1.0000 & 0.9610 & 0.1998 & 0.9610 & 1.0000 & 1.0000 & 1.0000 & 1.0000 & 1.0000 \\
\hline 375 & 1.0000 & 1.0000 & 1.0000 & 1.0000 & 1.0000 & 0.9727 & 0.1998 & 0.9727 & 1.0000 & 1.0000 & 1.0000 & 1.0000 & 1.0000 \\
\hline 400 & 1.0000 & 1.0000 & 1.0000 & 1.0000 & 1.0000 & 0.9814 & 0.1998 & 0.9814 & 1.0000 & 1.0000 & 1.0000 & 1.0000 & 1.0000 \\
\hline 425. & 1.0000 & 1.0000 & 1.0000 & 1.0000 & 1.0000 & 0.9878 & 0.1998 & 0.9878 & 1.0000 & 1.0000 & 1.0000 & 1.0000 & 1.0000 \\
\hline 450 & 1.0000 & 1.0000 & 1.0000 & 1.0000 & 1.0000 & 0.9922 & 0.1998 & 0.9922 & 1.0000 & 1.0000 & 1.0000 & 1.0000 & 1.0000 \\
\hline 475. & 1.0000 & 1.0000 & 1.0000 & 1.0000 & 1.0000 & 0.9953 & 0.1998 & 0.9953 & 1.0000 & 1.0000 & 1.0000 & 1.0000 & 1.0000 \\
\hline 500. & 1.0000 & 1.0000 & 1.0000 & 1.0000 & 1.0000 & .9972 & 0.1998 & 0.9972 & 1.0000 & 1.0000 & 1.0000 & 1.0000 & 1.0000 \\
\hline
\end{tabular}


TABI,E A.11 HIGII-RISE AFAKTMLENTS

DUE POWER OF THE TEST OF THE HY TOTIESIS TIAT MX = MO WIEN THE SIGNIHICANCE LEVEL IS 0.10

\begin{tabular}{|c|c|c|c|c|c|c|c|c|c|c|c|c|c|}
\hline \multirow[b]{2}{*}{$\mathrm{N}$} & \multicolumn{13}{|c|}{$(M X-M 0)$} \\
\hline & -15.00 & -12.50 & -10.00 & -7.50 & -5.00 & -2.50 & 0.00 & 2.50 & 5.00 & 7.50 & 10.00 & 12.50 & 15.00 \\
\hline 25 . & 0.9927 & 0.9610 & 0.8602 & 0.6554 & 0.3903 & 0.1777 & 0.1000 & 0.1777 & 0.3903 & 0.6554 & 0.8602 & 0.9610 & 0.9927 \\
\hline 50 . & 1.0000 & 0.9994 & 0.9879 & 0.8998 & 0.6200 & 0.2559 & 0.1000 & 0.2559 & 0.6200 & 0.8998 & 0.9879 & 0,9994 & 1.0000 \\
\hline 75. & 1.0000 & 1.0000 & 0.9993 & 0.9762 & 0.7800 & 0.3334 & 0.1000 & 0.3334 & 0.7800 & 0.9762 & 0.9993 & 1.0000 & 1.0000 \\
\hline 100. & 1.0000 & 1.0000 & 1.0000 & 0.9952 & 0.8810 & 0.4092 & 0.1000 & 0.4092 & 0.8810 & 0.9952 & 1.0000 & 1.0000 & 1.0000 \\
\hline 125. & 1.0000 & 1.0000 & 1.0000 & 0.9992 & 0.9398 & 0.4823 & 0.1000 & 0.4823 & 0.9398 & 0.9992 & 1.0000 & 1.0000 & 1.0000 \\
\hline 150 . & 1.0000 & 1.0000 & 1.0000 & 0.9999 & 0.9715 & 0.5517 & 0.1000 & 0.5517 & 0.9715 & 0.9999 & 1.0000 & 1.0000 & 1.0000 \\
\hline 175. & 1.0000 & 1.0000 & 1.0000 & 1.0000 & 0.9874 & 0.6168 & 0.1000 & 0.6168 & 0.9874 & 1.0000 & 1.0000 & 1.0000 & 1.0000 \\
\hline 200 . & 1.0000 & 1.0000 & 1.0000 & 1.0000 & 0.9948 & 0.6770 & 0.1000 & 0.6770 & 0.9948 & 1.0000 & 1.0000 & 1.0000 & 1.0000 \\
\hline 225 . & 1.0000 & 1.0000 & 1.0000 & 1.0000 & 0.9980 & 0.7317 & 0.1000 & 0.7317 & 0.9980 & 1.0000 & 1.0000 & 1.0000 & 1.0000 \\
\hline 250. & 1.0000 & 1.0000 & 1.0000 & 1.0000 & 0.9993 & 0.7807 & 0.1000 & 0.7807 & 0.9993 & 1.0000 & 1.0000 & 1.0000 & 1.0000 \\
\hline 275 . & 1.0000 & 1.0000 & 1.0000 & 1.0000 & 0.9998 & 0.8238 & 0.1000 & 0.8238 & 0.9998 & 1.0000 & 1.0000 & 1.0000 & 1.0000 \\
\hline 300 . & 1.0000 & 1.0000 & 1.0000 & 1.0000 & 0.9999 & 0.8611 & 0.1000 & 0.8611 & 0.9999 & 1.0000 & 1.0000 & 1.0000 & 1.0000 \\
\hline 325. & 1.0000 & 1.0000 & 1.0000 & 1.0000 & 1.0000 & 0.8928 & 0.1000 & 0.8928 & 1.0000 & 1.0000 & 1.0000 & 1.0000 & 1.0000 \\
\hline 350. & 1.0000 & 1.0000 & 1.0000 & 1.0000 & 1.0000 & 0.9191 & 0.1000 & 0.9191 & 1.0000 & 1.0000 & 1.0000 & 1.0000 & 1.0000 \\
\hline 375. & 1.0000 & 1.0000 & 1.0000 & 1.0000 & 1.0000 & 0.9405 & 0.1000 & 0.9405 & 1.0000 & 1.0000 & 1.0000 & 1.0000 & 1.0000 \\
\hline 400 . & 1.0000 & 1.0000 & 1.0000 & 1.0000 & 1.0000 & 0.9574 & 0.1000 & 0.9574 & 1.0000 & 1.0000 & 1.0000 & 1.0000 & 1.0000 \\
\hline 425. & 1.0000 & 1.0000 & 1.0000 & 1.0000 & 1.0000 & 0.9704 & 0.1000 & 0.9704 & 1.0000 & 1.0000 & 1.0000 & 1.0000 & 1.0000 \\
\hline 450 . & 1.0000 & 1.0000 & 1.0000 & 1.0000 & 1.0000 & 0.9802 & 0.1000 & 0.9802 & 1.0000 & 1.0000 & 1.0000 & 1.0000 & 1.0000 \\
\hline 475 . & 1.0000 & 1.0000 & 1.0000 & 1.0000 & 1.0000 & 0.9872 & 0.1000 & 0.9872 & 1.0000 & 1.0000 & 1.0000 & 1.0000 & 1.0000 \\
\hline 500. & 1.0000 & 1.0000 & 1.0000 & 1.0000 & 1.0000 & 0.9921 & 0.1000 & 0.9921 & 1.0000 & 1.0000 & 1.0000 & 1.0000 & 1.0000 \\
\hline
\end{tabular}


TABLE A.12 III (HI-RISE AHARTMENTS

THE POHER OF THE TEST OF THE HYPOTIESIS THAT MX = HO WILN THE SIGNILICANCE LFVEL IS 0.05

\begin{tabular}{|c|c|c|c|c|c|c|c|c|c|c|c|c|c|}
\hline \multirow[b]{2}{*}{$\mathbf{N}$} & \multicolumn{13}{|c|}{$(M X-M O)$} \\
\hline & -15.00 & -12.50 & -10.00 & -7.50 & -5.00 & -2.50 & 0.00 & 2.50 & 5.00 & 7.50 & 10.00 & 12.50 & 15.00 \\
\hline 25. & 0.9834 & 0.9261 & 0.7782 & 0.5337 & 0.2757 & 0.1047 & 0.0500 & 0.1047 & 0.2757 & 0.5337 & 0.7782 & 0.9261 & 0.9834 \\
\hline 50 . & 0.9999 & 0.9982 & 0.9738 & 0.8328 & 0.4961 & 0.1640 & 0.0500 & 0.1640 & 0.4961 & 0.8328 & 0.9738 & 0.9982 & 0.9999 \\
\hline 75. & 1.0000 & 1.0000 & 0.9980 & 0.9521 & 0.6762 & 0.2269 & 0.0500 & 0.2269 & 0.6762 & 0.9521 & 0.9980 & 1.0000 & 1.0000 \\
\hline 100. & 1.0000 & 1.0000 & 0.9999 & 0.9886 & 0.8065 & 0.2924 & 0.0500 & 0.2924 & 0.8065 & 0.9886 & 0.9999 & 1.0000 & 1.0000 \\
\hline 125. & 1.0000 & 1.0000 & 1.0000 & 0.9977 & 0.8922 & 0.3593 & 0.0500 & 0.3593 & 0.8922 & 0.9977 & 1.0000 & 1.0000 & 1.0000 \\
\hline 150. & 1.0000 & 1.0000 & 1.0000 & 0.9996 & 0.9439 & 0.4264 & 0.0500 & 0.4264 & 0.9439 & 0.9996 & 1.0000 & 1.0000 & 1.0000 \\
\hline 175. & 1.0000 & 1.0000 & 1.0000 & 0.9999 & 0.9728 & 0.4928 & 0.0500 & 0.4928 & 0.9728 & 0.9999 & 1.0000 & 1.0000 & 1.0040 \\
\hline 200 . & 1.0000 & 1.0000 & 1.0000 & 1.0000 & 0.9877 & 0.5573 & 0.0500 & 0.5573 & 0.9877 & 1.0000 & 1.0000 & 1.0000 & 1.0000 \\
\hline 225 . & 1.0000 & 1.0000 & 1.0000 & 1.0000 & 0.9948 & 0.6190 & 0.0500 & 0.6190 & 0.9948 & 1.0000 & 1.0000 & 1.0000 & 1.0000 \\
\hline 250. & 1.0000 & 1.0000 & 1.0000 & 1.0000 & 0.9980 & 0.6770 & 0.0500 & 0.6770 & 0.9980 & 1.0000 & 1.0000 & 1.0000 & 1.0000 \\
\hline 275. & 1.0000 & 1.0000 & 1.0000 & 1.0000 & 0.9993 & 0.7307 & 0.0500 & 0.7307 & 0.9943 & 1.0000 & 1.0000 & 1.0000 & 1.0000 \\
\hline 300. & 1.0000 & 1.0000 & 1.0000 & 1.0000 & 0.9998 & 0.7794 & 0.0500 & 0.7794 & 0.9998 & 1.0000 & 1.0000 & 1.0000 & 1.0000 \\
\hline 325 . & 1.0000 & 1.0000 & 1.0000 & 1.0000 & 0.9999 & 0.8229 & 0.0500 & 0.8229 & 0.9999 & 1.0000 & 1.0000 & 1.0000 & 1.0000 \\
\hline 350. & 1.0000 & 1.0000 & 1.0000 & 1.0000 & 1.0000 & 0.8608 & 0.0500 & 0.8608 & 1.0000 & 1.0000 & 1.0000 & 1.0000 & 1.0000 \\
\hline 375. & 1.0000 & 1.0000 & 1.0000 & 1.0000 & 1.0000 & 0.8932 & 0.0500 & 0.8932 & 1.0000 & 1.0000 & 1.0000 & 1.0000 & 1.0000 \\
\hline 400. & 1.0000 & 1.0000 & 1.0000 & 1.0000 & 1.0000 & 0.9201 & 0.0500 & 0.9201 & 1.0000 & 1.0000 & 1.0000 & 1.0000 & 1.0000 \\
\hline 425 . & 1.0000 & 1.0000 & 1.0000 & 1.0000 & 1.0000 & 0.9420 & 0.0500 & 0.9420 & 1.0000 & 1.0000 & 1.0000 & 1.0000 & 1.0000 \\
\hline 450 . & 1.0000 & 1.0000 & 1.0000 & 1.0000 & 1.0000 & 0.9592 & 0.0500 & 0.9592 & 1.0000 & 1.0000 & 1.0000 & 1.0000 & 1.0000 \\
\hline 475 . & 1.0000 & 1.0000 & 1.0000 & 1.0000 & 1.0000 & 0.9724 & 0.0500 & 0.9724 & 1.0000 & 1.0000 & 1.0000 & 1.0000 & 1.0000 \\
\hline 500 。 & 1.0000 & 1.0000 & 1.0000 & 1,0000 & 1.0000 & 0.9820 & 0.0500 & 0.9820 & 1.0000 & 1.0000 & 1.0000 & 1.0000 & 1.0000 \\
\hline
\end{tabular}


TAHLE A.13 ELEAENTARY SCHOOL

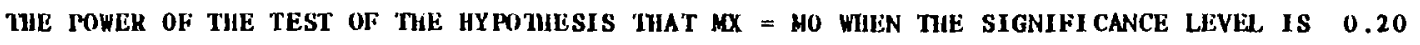

\begin{tabular}{|c|c|c|c|c|c|c|c|c|c|c|c|c|c|}
\hline \multirow[b]{2}{*}{$N$} & \multicolumn{13}{|c|}{$(M X-M 0)$} \\
\hline & -15.00 & -12.50 & -10.00 & -7.50 & -5.00 & -2.50 & 0.00 & 2.50 & 5.00 & 7.50 & 10.00 & 12.50 & 15.00 \\
\hline 18. & 0.9964 & 0.9786 & 0.9137 & 0.7593 & 0.5209 & 0.2934 & 0.1998 & 0.2934 & 0.5209 & 0.7593 & 0.9137 & 0.9786 & 0.9964 \\
\hline 36. & 1.0000 & 0.9998 & 0.9947 & 0.9451 & 0.7391 & 0.3863 & 0.1998 & 0.3863 & 0.7391 & 0.9451 & 0.9947 & 0.9998 & 1.0000 \\
\hline 54. & 1.0000 & 1.0000 & 0.9998 & 0.9905 & 0.8721 & 0.4772 & 0.1998 & 0.4772 & 0.8721 & 0.9905 & 0.9998 & 1.0000 & 1.0000 \\
\hline 72. & 1.0000 & 1.0000 & 1.0000 & 0.9988 & 0.9444 & 0.5650 & 0.1998 & 0.5650 & 0.9444 & 0.9988 & 1.0000 & 1.0000 & 1.0000 \\
\hline 90 . & 1.0000 & 1.0000 & 1.0000 & 0.9999 & 0.9790 & 0.6481 & 0.1998 & 0.6481 & 0.9790 & 0.9999 & 1.0000 & 1.0000 & 1.0000 \\
\hline 108. & 1.0000 & 1.0000 & 1.0000 & 1.0000 & 0.9933 & 0.7249 & 0.1998 & 0.7249 & 0.9933 & 1.0000 & 1.0000 & 1.0000 & 1.0000 \\
\hline 126. & 1.0000 & 1.0000 & 1.0000 & 1.0000 & 0.9982 & 0.7938 & 0.1998 & 0.7938 & 0.9982 & 1.0000 & 1.0000 & 1.0000 & 1.0000 \\
\hline 144. & 1.0000 & 1.0000 & 1.0000 & 1.0000 & 0.9996 & 0.8535 & 0.1998 & 0.8535 & 0.9996 & 1.0000 & 1.0000 & 1.0000 & 1.0000 \\
\hline 162. & 1.0000 & 1.0000 & 1.0000 & 1.0000 & 0.9999 & 0.9027 & 0.1998 & 0.9027 & 0.9999 & 1.0000 & 1.0000 & 1.0000 & 1.0000 \\
\hline 180. & 1.0000 & 1.0000 & 1.0000 & 1.0000 & 1.0000 & 0.9408 & 0.1998 & 0.9408 & 1.0000 & 1.0000 & 1.0000 & 1.0000 & 1.0000 \\
\hline 198. & 1.0000 & 1.0000 & 1.0000 & 1.0000 & 1.0000 & 0.9680 & 0.1998 & 0.9680 & 1.0000 & 1.0000 & 1.0000 & 1.0000 & 1.0000 \\
\hline 216. & 1.0000 & 1.0000 & 1.0000 & 1.0000 & 1.0000 & 0.9852 & 0.1998 & 0.9852 & 1.0000 & 1.0000 & 1.0000 & 1.0000 & 1.0000 \\
\hline 234 . & 1.0000 & 1.0000 & 1.0000 & 1.0000 & 1.0000 & 0.9946 & 0.1998 & 0.9946 & 1.0000 & 1.0000 & 1.0000 & 1.0000 & 1.0000 \\
\hline 252 . & 1.0000 & 1.0000 & 1.0000 & 1.0000 & 1.0000 & 0.9986 & 0.1998 & 0.9986 & 1.0000 & 1.0000 & 1.0000 & 1.0000 & 1.0000 \\
\hline 270. & 1.0000 & 1.0000 & 1.0000 & 1.0000 & 1.0000 & 0.9998 & 0.1998 & 0.9998 & 1.0000 & 1.0000 & 1.0000 & 1.0000 & 1.0000 \\
\hline 288 . & 1.0000 & 1.0000 & 1.0000 & 1.0000 & 1.0000 & 1.0000 & 0.1998 & 1.0000 & 1.0000 & 1.0000 & 1.0000 & 1.0000 & 1.0000 \\
\hline 306. & 1.0000 & 1.0000 & 1.0000 & 1.0000 & 1.0000 & 1.0000 & 0.1998 & 1.0000 & 1.0000 & 1.0000 & 1.0000 & 1.0000 & 1.0000 \\
\hline 324. & 1.0000 & 1.0000 & 1.0000 & 1.0000 & 1.0000 & 1.0000 & 0.1998 & 1.0000 & 1.0000 & 1.0000 & 1.0000 & 1.0000 & 1.0000 \\
\hline 342 . & 1.0000 & 1.0000 & 1.0000 & 1.0000 & 1.0000 & 1.0000 & 0.1998 & 1.0000 & 1.0000 & 1.0000 & 1.0000 & 1.0000 & 1.0000 \\
\hline 360 . & 1.0000 & 1.0000 & 1.0000 & 1.0000 & 1.0000 & 1.0000 & 0,1998 & 1.0000 & 1.0000 & 1.0000 & 1.0000 & 1.0000 & 1.0000 \\
\hline
\end{tabular}


TABLE A.14 EJ,EHENTAKY SCHUOL,

THE POWER OF THE TEST OF THE HYPO'HESIS IIIAT MX $=$ MO WHUN MHE SIGNIFICANCE LEVUL IS 0.10

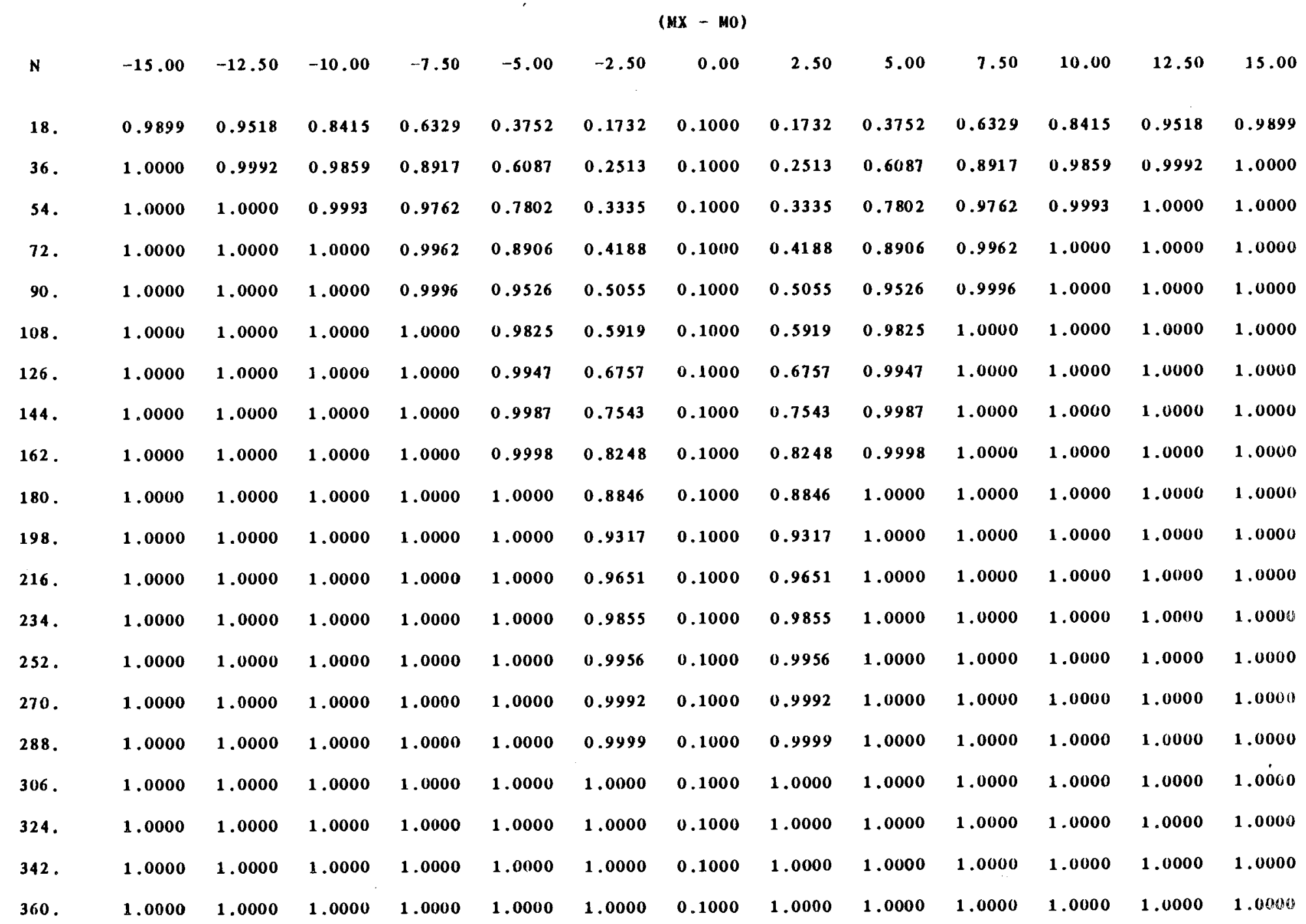


TABLE A.15 FLEMENTARY SCHOOL

TUE POWER OF TIIE TEST OF TIE HYPOTHESIS THAT MX = MO WHEN THE SIGNIFI CANCE LEVEL $1 S$ S 0.05

\begin{tabular}{|c|c|c|c|c|c|c|c|c|c|c|c|c|c|}
\hline \multirow[b]{2}{*}{$\mathbf{N}$} & \multicolumn{13}{|c|}{$(M X-M O)$} \\
\hline & -15.00 & -12.50 & -10.00 & -7.50 & -5.00 & -2.50 & 0.00 & 2.50 & 5.00 & 7.50 & 10.00 & 12.50 & 15.00 \\
\hline 18. & 0.9777 & 0.9110 & 0.7535 & 0.5097 & 0.2625 & 0.1014 & 0.0500 & 0.1014 & 0.2625 & 0.5097 & 0.7535 & 0.9110 & 0.9777 \\
\hline 36. & 0.9999 & 0.9978 & 0.9700 & 0.8214 & 0.4842 & 0.1604 & 0.0500 & 0.1604 & 0.4842 & 0.8214 & 0.9700 & 0.9978 & 0.9999 \\
\hline 54. & 1.0000 & 1.0000 & 0.9980 & 0.9522 & 0.6765 & 0.2271 & 0.0500 & 0.2271 & 0.6765 & 0.9522 & 0.9980 & 1.0000 & 1.0000 \\
\hline 72. & 1.0000 & 1.0000 & 0.9999 & 0.9907 & 0.8198 & 0.3009 & 0.0500 & 0.3009 & 0.8198 & 0.9907 & 0.9999 & 1.0000 & 1.0000 \\
\hline 90. & 1.0000 & 1.0000 & 1.0000 & 0.9987 & 0.9123 & 0.3813 & 0.0500 & 0.3813 & 0.9123 & 0.9987 & 1.0000 & 1.0000 & 1.0000 \\
\hline 108 . & 1.0000 & 1.0000 & 1.0000 & 0.9999 & 0.9636 & 0.4670 & 0.0500 & 0.4670 & 0.9636 & 0.9999 & 1.0000 & 1.0000 & 1.0000 \\
\hline 126. & 1.0000 & 1.0000 & 1.0000 & 1.0000 & 0.9875 & 0.5559 & 0.0500 & 0.5559 & 0.9875 & 1.0000 & 1.0000 & 1.0000 & 1.0000 \\
\hline 144. & 1.0000 & 1.0000 & 1.0000 & 1.0000 & 0.9966 & 0.6454 & 0.0500 & 0.6454 & 0.9966 & 1.0000 & 1.0000 & 1.0000 & 1.0000 \\
\hline 162. & 1.0000 & 1.0000 & 1.0000 & 1.0000 & 0.9993 & 0.7320 & 0.0500 & 0.7320 & 0.9993 & 1.0000 & 1.0000 & 1.0000 & 1.0000 \\
\hline 180. & 1.0000 & 1.0000 & 1.0000 & 1.0000 & 0.9999 & 0.8115 & 0.0500 & 0.8115 & 0.9999 & 1.0000 & 1.0000 & 1.0000 & 1.0000 \\
\hline 198. & 1.0000 & 1.0000 & 1.0000 & 1.0000 & 1.0000 & 0.8797 & 0.0500 & 0.8797 & 1.0000 & 1.0000 & 1.0000 & 1.0000 & 1.0000 \\
\hline 216. & 1.0000 & 1.0000 & 1.0000 & 1.0000 & 1.0000 & 0.9329 & 0.0500 & 0.9329 & 1.0000 & 1.0000 & 1.0000 & 1.0000 & 1.0000 \\
\hline 234 & 1.0000 & 1.0000 & 1.0000 & 1.0000 & 1.0000 & 0.9691 & 0.0500 & 0.9691 & 1.0000 & 1.0000 & 1.0000 & 1.0000 & 1.0000 \\
\hline 252. & 1.0000 & 1.0000 & 1.0000 & 1.0000 & 1.0000 & 0.9893 & 0.0500 & 0.9893 & 1.0000 & 1.0000 & 1.0000 & 1.0000 & 1.0000 \\
\hline 270. & 1.0000 & 1.0000 & 1.0000 & 1.0000 & 1.0000 & 0.9977 & 0.0500 & 0.9977 & 1.0000 & 1.0000 & 1.0000 & 1.0000 & 1.0000 \\
\hline 288. & 1.0000 & 1.0000 & 1.0000 & 1.0000 & 1.0000 & 0.9998 & 0.0500 & 0.9998 & 1.0000 & 1.0000 & 1.0000 & 1.0000 & 1.0000 \\
\hline 306. & 1.0000 & 1.0000 & 1.0000 & 1.0000 & 1.0000 & 1.0000 & 0.0500 & 1.0000 & 1.0000 & 1.0000 & 1.0000 & 1.0000 & 1.0000 \\
\hline 324. & 1.0000 & 1.0000 & 1.0000 & 1.0000 & 1.0000 & 1.0000 & 0.0500 & 1.0000 & 1.0000 & 1.0000 & 1.0000 & 1.0000 & 1.0000 \\
\hline 342 . & 1.0000 & 1.0000 & 1.0000 & 1.0000 & 1.0000 & 1.0000 & 0.0500 & 1.0000 & 1.0000 & 1.0000 & 1.0000 & 1.0000 & 1.0000 \\
\hline 60 . & 1.0000 & 1.0000 & 1.0000 & 1.0000 & 1.0000 & 1.0000 & 0.0500 & 1.0000 & 1.0000 & 1.0000 & 1.0000 & 1.0000 & 1.0000 \\
\hline
\end{tabular}


TABLE A.16 SLICONDARY SCHOOL

THE POHER OF THE TEST OF TIE IYYO'IIESIS THIAT MX = MO WIEN THE SIGNIFICANCE LEVEL IS 0.20

\begin{tabular}{|c|c|c|c|c|c|c|c|c|c|c|c|c|c|}
\hline \multirow[b]{2}{*}{$\mathbf{N}$} & \multicolumn{13}{|c|}{$(M X-M 0)$} \\
\hline & -15.00 & -12.50 & -10.00 & -7.50 & -5.00 & -2.50 & 0.00 & 2.50 & 5.00 & 7.50 & 10.00 & 12.50 & 15.00 \\
\hline 25 . & 0.9160 & 0.8254 & 0.6897 & 0.5238 & 0.3614 & 0.2431 & 0.1998 & 0.2431 & 0.3614 & 0.5238 & 0.6897 & 0.8254 & 0.9160 \\
\hline 50. & 0.9949 & 0.9729 & 0.9003 & 0.7402 & 0.5054 & 0.2881 & 0.1998 & 0.2881 & 0.5054 & 0.7402 & 0.9003 & 0.9729 & 0.9949 \\
\hline 75. & 0.9998 & 0.9969 & 0.9735 & 0.8709 & 0.6298 & 0.3348 & 0.1998 & 0.3348 & 0.6298 & 0.8709 & 0.9735 & 0.9969 & 0.9998 \\
\hline 100. & 1.0000 & 0.9997 & 0.9942 & 0.9421 & 0.7335 & 0.3833 & 0.1998 & 0.3833 & 0.7335 & 0.9421 & 0.9942 & 0.9997 & 1.0000 \\
\hline 125. & 1.0000 & 1.0000 & 0.9990 & 0.9769 & 0.8166 & 0.4334 & 0.1998 & 0.4334 & 0.8166 & 0.9769 & 0.9990 & 1.0000 & 1.0000 \\
\hline 150. & 1.0000 & 1.0000 & 0.9999 & 0.9919 & 0.8803 & 0.4849 & 0.1998 & 0.4849 & 0.8803 & 0.9919 & 0.9999 & 1.0000 & 1.0000 \\
\hline 175. & 1.0000 & 1.0000 & 1.0000 & 0.9976 & 0.9266 & 0.5376 & 0.1998 & 0.5376 & 0.9266 & 0.9976 & 1.0000 & 1.0000 & 1.0000 \\
\hline 200. & 1.0000 & 1.0000 & 1.0000 & 0.9994 & 0.9582 & 0.5913 & 0.1998 & 0.5913 & 0.9582 & 0.9994 & 1.0000 & 1.0000 & 1.0000 \\
\hline 225. & 1.0000 & 1.0000 & 1.0000 & 0.9999 & 0.9782 & 0.6454 & 0.1998 & 0.6454 & 0.9782 & 0.9999 & 1.0000 & 1.0000 & 1.0000 \\
\hline 250. & 1.0000 & 1.0000 & 1.0000 & 1.0000 & 0.9899 & 0.6993 & 0.1998 & 0.6993 & 0.9899 & 1.0000 & 1.0000 & 1.0000 & 1.0000 \\
\hline 275. & 1.0000 & 1.0000 & 1.0000 & 1.0000 & 0.9959 & 0.7522 & 0.1998 & 0.7522 & 0.9959 & 1.0000 & 1.0000 & 1.0000 & 1.0000 \\
\hline 300. & 1.0000 & 1.0000 & 1.0000 & 1.0000 & 0.9986 & 0.8031 & 0.1998 & 0.8031 & 0.9986 & 1.0000 & 1.0000 & 1.0000 & 1.0000 \\
\hline 325. & 1.0000 & 1.0000 & 1.0000 & 1.0000 & 0.9996 & 0.8507 & 0.1998 & 0.8507 & 0.9996 & 1.0000 & 1.0000 & 1.0000 & 1.0000 \\
\hline 350. & 1.0000 & 1.0000 & 1.0000 & 1.0000 & 0.9999 & 0.8936 & 0.1998 & 0.8936 & 0.9999 & 1.0000 & 1.0000 & 1.0000 & 1.0000 \\
\hline 375. & 1.0000 & 1.0000 & 1.0000 & 1.0000 & 1.0000 & 0.9304 & 0.1998 & 0.9304 & 1.0000 & 1.0000 & 1.0000 & 1.0000 & 1.0000 \\
\hline 400 & 1.0000 & 1.0000 & 1.0000 & 1.0000 & 1.0000 & 0.9596 & 0.1998 & 0.9596 & 1.0000 & 1.0000 & 1.0000 & 1.0000 & 1.0000 \\
\hline 425. & 1.0000 & 1.0000 & 1.0000 & 1.0000 & 1.0000 & 0.9803 & 0.1998 & 0.9803 & 1.0000 & 1.0000 & 1.0000 & 1.0000 & 1.0000 \\
\hline 450 . & 1.0000 & 1.0000 & 1.0000 & 1.0000 & 1.0000 & 0.9926 & 0.1998 & 0.9926 & 1.0000 & 1.0000 & 1.0000 & 1.0000 & 1.0000 \\
\hline 475 . & 1.0000 & 1.0000 & 1.0000 & 1.0000 & 1.0000 & 0.9982 & 0.1998 & 0.9982 & 1.0000 & 1.0000 & 1.0000 & 1.0000 & 1.0000 \\
\hline 500. & 1.0000 & 1.0000 & 1.0000 & 1.0000 & 1.0000 & 0.9998 & 0.1998 & 0.9998 & 1.0000 & 1.0000 & 1.0000 & 1.0000 & 1.0000 \\
\hline
\end{tabular}


TABLE A.17 SECONDARY SCIOOL

THE YOWER UF THE TEST OF THE IIYTOTHESIS THAT MX = HO WILN THE SIGNINICANCE JEVEL IS 0.10

\begin{tabular}{|c|c|c|c|c|c|c|c|c|c|c|c|c|c|}
\hline \multirow[b]{2}{*}{$\mathbf{N}$} & \multicolumn{13}{|c|}{$(M X-M 0)$} \\
\hline & -15.00 & -12.50 & -10.00 & -7.50 & -5.00 & -2.50 & 0.00 & 2.50 & 5.00 & 7.50 & 10.00 & 12.50 & 15.00 \\
\hline 25 . & 0.8451 & 0.7164 & 0.5515 & 0.3780 & 0.2299 & 0.1331 & 0.1000 & 0.1331 & 0.2299 & 0.3780 & 0.5515 & 0.7164 & 0.8451 \\
\hline 50. & 0.9862 & 0.9408 & 0.8213 & 0.6100 & 0.3602 & 0.1689 & 0.1000 & 0.1689 & 0.3602 & 0.6100 & 0.8213 & 0.9408 & 0.9862 \\
\hline 75. & 0.9993 & 0.9912 & 0.9419 & 0.7785 & 0.4859 & 0.2073 & 0.1000 & 0.2073 & 0.4859 & 0.7785 & 0.9419 & 0.9912 & 0.9993 \\
\hline 100. & 1.0000 & 0.9991 & 0.9847 & 0.8868 & 0.6020 & 0.2487 & 0.1000 & 0.2487 & 0.6020 & 0.8868 & 0.9847 & 0.9991 & 1.0000 \\
\hline 125. & 1.0000 & 0.9999 & 0.9968 & 0.9485 & 0.7049 & 0.2931 & 0.1000 & 0.2931 & 0.7049 & 0.9485 & 0.9968 & 0.9999 & 1.0000 \\
\hline 150. & 1.0000 & 1.0000 & 0.9995 & 0.9794 & 0.7919 & 0.3407 & 0.1000 & 0.3407 & 0.7919 & 0.9794 & 0.9995 & 1.0000 & 1.0000 \\
\hline 175. & 1.0000 & 1.0000 & 0.9999 & 0.9929 & 0.8616 & 0.3915 & 0.1000 & 0.3915 & 0.8616 & 0.9929 & 0.9999 & 1.0000 & 1.0000 \\
\hline 200 & 1.0000 & 1.0000 & 1.0000 & 0.9980 & 0.9142 & 0.4456 & 0.1000 & 0.4456 & 0.9142 & 0.9980 & 1.0000 & 1.0000 & 1.0000 \\
\hline 225 . & 1.0000 & 1.0000 & 1.0000 & 0.9995 & 0.9511 & 0.5026 & 0.1000 & 0.5026 & 0.9511 & 0.9995 & 1.0000 & 1.0000 & 1.0000 \\
\hline 250 . & 1.0000 & 1.0000 & 1.0000 & 0.9999 & 0.9749 & 0.5624 & 0.1000 & 0.5624 & 0.9749 & 0.9999 & 1.0000 & 1.0000 & 1.0000 \\
\hline 275 . & 1.0000 & 1.0000 & 1.0000 & 1.0000 & 0.9886 & 0.6243 & 0.1000 & 0.6243 & 0.9886 & 1.0000 & 1.0000 & 1.0000 & 1.0000 \\
\hline 300. & 1.0000 & 1.0000 & 1.0000 & 1.0000 & 0.9956 & 0.6875 & 0.1000 & 0.6875 & 0.9956 & 1.0000 & 1.0000 & 1.0000 & 1.0000 \\
\hline 325. & 1.0000 & 1.0000 & 1.0000 & 1.0000 & 0.9986 & 0.7504 & 0.1000 & 0.7504 & 0.9986 & 1.0000 & 1.0000 & 1.0000 & 1.0000 \\
\hline 350. & 1.0000 & 1.0000 & 1.0000 & 1.0000 & 0.9997 & 0.8113 & 0.1000 & 0.8113 & 0.9997 & 1.0000 & 1.0000 & 1.0000 & 1.0000 \\
\hline 375 . & 1.0000 & 1.0000 & 1.0000 & 1.0000 & 0.9999 & 0.8677 & 0.1000 & 0.8677 & 0.9999 & 1.0000 & 1.0000 & 1.0000 & 1.0000 \\
\hline 400 & 1.0000 & 1.0000 & 1.0000 & 1.0000 & 1.0000 & 0.9166 & 0.1000 & 0.9166 & 1.0000 & 1.0000 & 1.0000 & 1.0000 & 1.0000 \\
\hline 425. & 1.0000 & 1.0000 & 1.0000 & 1.0000 & 1.0000 & 0.9551 & 0.1000 & 0.9551 & 1.0000 & 1.0000 & 1.0000 & 1.0000 & 1.0000 \\
\hline 450. & 1.0000 & 1.0000 & 1.0000 & 1.0000 & 1.0000 & 0.9810 & 0.1000 & 0.9810 & 1.0000 & 1.0000 & 1.0000 & 1.0000 & 1.0000 \\
\hline 475. & 1.0000 & 1.0000 & 1.0000 & 1.0000 & 1.0000 & 0.9947 & 0.1000 & 0.9947 & 1.0000 & 1.0000 & 1.0000 & 1.0000 & 1.0000 \\
\hline 500. & 1.0000 & 1.0000 & 1.0000 & 1.0000 & 1.0000 & 0.9993 & 0.1000 & 0.9993 & 1.0000 & 1.0000 & 1.0000 & 1.0000 & 1.0000 \\
\hline
\end{tabular}


TABLE: A.18 SLCONBARY SCHOOL

THE POWER OF THE TEST OF THLi HY FO MUESIS THAT MX = MO WHEN THL SIGNIFICANCE LEVE IS 0.05

\begin{tabular}{|c|c|c|c|c|c|c|c|c|c|c|c|c|c|}
\hline \multirow[b]{2}{*}{$\mathbf{N}$} & \multicolumn{13}{|c|}{$(M X-M 0)$} \\
\hline & -15.00 & -12.50 & -10.00 & -7.50 & -5.00 & -2.50 & 0.00 & 2.50 & 5.00 & 7.50 & 10.00 & 12.50 & 15.00 \\
\hline 25. & 0.7582 & 0.6015 & 0.4262 & 0.2649 & 0.1438 & 0.0728 & 0.0500 & 0.0728 & 0.1438 & 0.2649 & 0.4262 & 0.6015 & 0.7582 \\
\hline 50. & 0.9705 & 0.8937 & 0.7275 & 0.4856 & 0.2497 & 0.0983 & 0.0500 & 0.0983 & 0.2497 & 0.4856 & 0.7275 & 0.8937 & 0.9705 \\
\hline 75. & 0.9979 & 0.9803 & 0.8955 & 0.6744 & 0.3626 & 0.1267 & 0.0500 & 0.1267 & 0.3626 & 0.6744 & 0.8955 & 0.9803 & 0.9979 \\
\hline 100. & 0.9999 & 0.9974 & 0.9676 & 0.8145 & 0.4774 & 0.1584 & 0.0500 & 0.1584 & 0.4774 & 0.8145 & 0.9676 & 0.9974 & 0.9999 \\
\hline 125. & 1.0000 & 0.9998 & 0.9920 & 0.9058 & 0.5884 & 0.1938 & 0.0500 & 0.1938 & 0.5884 & 0.9058 & 0.9920 & 0.9998 & 1.0000 \\
\hline 150. & 1.0000 & 1.0000 & 0.9984 & 0.9579 & 0.6908 & 0.2331 & 0.0500 & 0.2331 & 0.6908 & 0.9579 & 0.9984 & 1.0000 & 1.0000 \\
\hline 175. & 1.0000 & 1.0000 & 0.9998 & 0.9838 & 0.7801 & 0.2768 & 0.0500 & 0.2768 & 0.7801 & 0.9838 & 0.9998 & 1.0000 & 1.0000 \\
\hline 200. & 1.0000 & 1.0000 & 1.0000 & 0.9947 & 0.8536 & 0.3252 & 0.0500 & 0.3252 & 0.8536 & 0.9947 & 1.0000 & 1.0000 & 1.0000 \\
\hline 225. & 1.0000 & 1.0000 & 1.0000 & 0.9986 & 0.9100 & 0.3786 & 0.0500 & 0.3786 & 0.9100 & 0.9986 & 1.0000 & 1.0000 & 1.0000 \\
\hline 250. & 1.0000 & 1.0000 & 1.0000 & 0.9997 & 0.9498 & 0.4371 & 0.0500 & 0.4371 & 0.9498 & 0.9997 & 1.0000 & 1.0000 & 1.0000 \\
\hline 275. & 1.0000 & 1.0000 & 1.0000 & 1.0000 & 0.9752 & 0.5006 & 0.0500 & 0.5006 & 0.9752 & 1.0000 & 1.0000 & 1.0000 & 1.0000 \\
\hline 300 & 1.0000 & 1.0000 & 1.0000 & 1.0000 & 0.9895 & 0.5689 & 0.0500 & 0.5689 & 0.9895 & 1.0000 & 1.0000 & 1.0000 & 1.0000 \\
\hline 325. & 1.0000 & 1.0000 & 1.0000 & 1.0000 & 0.9963 & 0.6409 & 0.0500 & 0.6409 & 0.9963 & 1.0000 & 1.0000 & 1.0000 & 1.0000 \\
\hline 350. & 1.0000 & 1.0000 & 1.0000 & 1.0000 & 0.9990 & 0.7149 & 0.0500 & 0.7149 & 0.9990 & 1.0000 & 1.0000 & 1.0000 & 1.0000 \\
\hline 375. & 1.0000 & 1.0000 & 1.0000 & 1.0000 & 0.9998 & 0.7883 & 0.0500 & 0.7883 & 0.9998 & 1.0000 & 1.0000 & 1.0000 & 1.0000 \\
\hline 400 & 1.0000 & 1.0000 & 1.0000 & 1.0000 & 1.0000 & 0.8571 & 0.0500 & 0.8571 & 1.0000 & 1.0000 & 1.0000 & 1.0000 & 1.0000 \\
\hline 425 . & 1.0000 & 1.0000 & 1.0000 & 1.0000 & 1.0000 & 0.9163 & 0.0500 & 0.9163 & 1.0000 & 1.0000 & 1.0000 & 1.0000 & 1.0000 \\
\hline 450 . & 1.0000 & 1.0000 & 1.0000 & 1.0000 & 1.0000 & 0.9608 & 0.0500 & 0.9608 & 1.0000 & 1.0000 & 1.0000 & 1.0000 & 1.0000 \\
\hline 475. & 1.0000 & 1.0000 & 1.0000 & 1.0000 & 1.0000 & 0.9874 & 0.0500 & 0.9874 & 1.0000 & 1.0000 & 1.0000 & 1.0000 & 1.0000 \\
\hline 500 & 1.0000 & 1.0000 & 1.0000 & 1.0000 & 1.0000 & 0.9980 & 0.0500 & 0.9980 & 1.0000 & 1.0000 & 1.0000 & 1.0000 & 1.0000 \\
\hline
\end{tabular}




\section{TABLE A.19 CLINIC}

THE POWLR OF THE TEST OF THE IYYU'HESIS THAT MX = MO WIEN THE SIGNIFICANCE LEVEL IS 0.20

\begin{tabular}{|c|c|c|c|c|c|c|c|c|c|c|c|c|c|}
\hline \multirow[b]{2}{*}{$\mathbf{N}$} & \multicolumn{13}{|c|}{$(M X-M 0)$} \\
\hline & -15.00 & -12.50 & -10.00 & -7.50 & -5.00 & -2.50 & 0.00 & 2.50 & 5.00 & 7.50 & 10.00 & 12.50 & 15.00 \\
\hline 25 . & 0.7239 & 0.6123 & 0.4926 & 0.3783 & 0.2838 & 0.2215 & 0.1998 & 0.2215 & 0.2838 & 0.3783 & 0.4926 & 0.6123 & 0.7239 \\
\hline 50 . & 0.9175 & 0.8274 & 0.6919 & 0.5256 & 0.3625 & 0.2434 & 0.1998 & 0.2434 & 0.3625 & 0.5256 & 0.6919 & 0.8274 & 0.9175 \\
\hline 75. & 0.9780 & 0.9284 & 0.8201 & 0.6444 & 0.4358 & 0.2654 & 0.1998 & 0.2654 & 0.4358 & 0.6444 & 0.8201 & 0.9284 & 0.9780 \\
\hline 100. & 0.9947 & 0.9722 & 0.8988 & 0.7381 & 0.5037 & 0.2875 & 0.1998 & 0.2875 & 0.5037 & 0.7381 & 0.8988 & 0.9722 & 0.9947 \\
\hline 125. & 0.9988 & 0.9898 & 0.9451 & 0.8105 & 0.5662 & 0.3098 & 0.1998 & 0.3098 & 0.5662 & 0.8105 & 0.9451 & 0.9898 & 0.9988 \\
\hline 150. & 0.9998 & 0.9965 & 0.9712 & 0.8653 & 0.6232 & 0.3321 & 0.1998 & 0.3321 & 0.6232 & 0.8653 & 0.9712 & 0.9965 & 0.9998 \\
\hline 175. & 1.0000 & 0.9988 & 0.9854 & 0.9059 & 0.6749 & 0.3545 & 0.1998 & 0.3545 & 0.6749 & 0.9059 & 0.9854 & 0.9988 & 1.0000 \\
\hline 200. & 1.0000 & 0.9996 & 0.9929 & 0.9355 & 0.7215 & 0.3771 & 0.1998 & 0.3771 & 0.7215 & 0.9355 & 0.9929 & 0.9996 & 1.0000 \\
\hline 225 . & 1.0000 & 0.9999 & 0.9967 & 0.9567 & 0.7631 & 0.3996 & 0.1998 & 0.3996 & 0.7631 & 0.9567 & 0.9967 & 0.9999 & 1.0000 \\
\hline 250. & 1.0000 & 1.0000 & 0.9985 & 0.9714 & 0.8001 & 0.4223 & 0.1998 & 0.4223 & 0.8001 & 0.9714 & 0.9985 & 1.0000 & 1.0000 \\
\hline 275 . & 1.0000 & 1.0000 & 0.9993 & 0.9815 & 0.8327 & 0.4449 & 0.1998 & 0.4449 & 0.8327 & 0.9815 & 0.9993 & 1.0000 & 1.0000 \\
\hline 300 . & 1.0000 & 1.0000 & 0.9997 & 0.9883 & 0.8611 & 0.4675 & 0.1998 & 0.4675 & 0.8611 & 0.9883 & 0.9997 & 1.0000 & 1.0000 \\
\hline 325. & 1.0000 & 1.0000 & 0.9999 & 0.9928 & 0.8857 & 0.4901 & 0.1998 & 0.4901 & 0.8857 & 0.9928 & 0.9999 & 1.0000 & 1.0000 \\
\hline 350. & 1.0000 & 1.0000 & 1.0000 & 0.9956 & 0.9068 & 0.5127 & 0.1998 & 0.5127 & 0.9068 & 0.9956 & 1.0000 & 1.0000 & 1.0000 \\
\hline 375. & 1.0000 & 1.0000 & 1.0000 & 0.9974 & 0.9248 & 0.5351 & 0.1998 & 0.5351 & 0.9248 & 0.9974 & 1.0000 & 1.0000 & 1.0000 \\
\hline 400. & 1.0000 & 1.0000 & 1.0000 & 0.9985 & 0.9399 & 0.5575 & 0.1998 & 0.5575 & 0.9399 & 0.9985 & 1.0000 & 1.0000 & 1.0000 \\
\hline 425. & 1.0000 & 1.0000 & 1.0000 & 0.9992 & 0.9525 & 0.5797 & 0.1998 & 0.5797 & 0.9525 & 0.9992 & 1.0000 & 1.0000 & 1.0000 \\
\hline 450 . & 1.0000 & 1.0000 & 1.0000 & 0.9995 & 0.9629 & 0.6017 & 0.1998 & 0.6017 & 0.9629 & 0.9995 & 1.0000 & 1.0000 & 1.0000 \\
\hline 475. & 1.0000 & 1.0000 & 1.0000 & 0.9998 & 0.9714 & 0.6236 & 0.1998 & 0.6236 & 0.9714 & 0.9998 & 1.0000 & 1.0000 & 1.0000 \\
\hline 500. & 1.0000 & 1.0000 & 1.0000 & 0.9999 & 0.9782 & 0.6452 & 0.1998 & 0.6452 & 0.9782 & 0.9999 & 1.0000 & 1.0000 & 1.0000 \\
\hline
\end{tabular}




\section{TABLE A.20 CLINIC}

THE POWEK OF THE TEST OF THE hYPOHESIS THAT MX = MO WHEN THE SIGNIFICANCE LEVEL IS 0.10

\begin{tabular}{|c|c|c|c|c|c|c|c|c|c|c|c|c|c|}
\hline $\mathbf{N}$ & -15.00 & -12.50 & -10.00 & -7.50 & -5.00 & -2.50 & 0.00 & 2.50 & 5.00 & 7.50 & 10.00 & 12.50 & 15.00 \\
\hline 25. & 0.5908 & 0.4674 & 0.3481 & 0.2444 & 0.1654 & 0.1165 & 0.1000 & 0.1165 & 0.1654 & 0.2444 & 0.3481 & 0.4674 & 0.5908 \\
\hline 50. & 0.8474 & 0.7191 & 0.5540 & 0.3797 & 0.2308 & 0.1334 & 0.1000 & 0.1334 & 0.2308 & 0.3797 & 0.5540 & 0.7191 & 0.8474 \\
\hline 75. & 0.9506 & 0.8646 & 0.7095 & 0.5015 & 0.2954 & 0.1507 & 0.1000 & 0.1507 & 0.2954 & 0.5015 & 0.7095 & 0.8646 & 0.9506 \\
\hline 100. & 0.9857 & 0.9395 & 0.8190 & 0.6075 & 0.3586 & 0.1684 & 0.1000 & 0.1684 & 0.3586 & 0.6075 & 0.8190 & 0.9395 & 0.9857 \\
\hline 125. & 0.9963 & 0.9748 & 0.8917 & 0.6970 & 0.4199 & 0.1865 & 0.1000 & 0.1865 & 0.4199 & 0.6970 & 0.8917 & 0.9748 & 0.9963 \\
\hline 150 . & 0.9991 & 0.9901 & 0.9377 & 0.7706 & 0.4789 & 0.2051 & 0.1000 & 0.2051 & 0.4789 & 0.7706 & 0.9377 & 0.9901 & 0.9991 \\
\hline 175. & 0.9998 & 0.9964 & 0.9655 & 0.8297 & 0.5350 & 0.2240 & 0.1000 & 0.2240 & 0.5350 & 0.8297 & 0.9655 & 0.9964 & 0.9998 \\
\hline 200 & 1.0000 & 0.9987 & 0.9816 & 0.8760 & 0.5880 & 0.2433 & 0.1000 & 0.2433 & 0.5880 & 0.8760 & 0.9816 & 0.9987 & 1.0000 \\
\hline 225 & 1.0000 & 0.9996 & 0.9906 & 0.9115 & 0.6376 & 0.2630 & 0.1000 & 0.2630 & 0.6376 & 0.9115 & 0.9906 & 0.9996 & 1.0000 \\
\hline 250 . & 1.0000 & 0.9999 & 0.9953 & 0.9381 & 0.6837 & 0.2832 & 0.1000 & 0.2832 & 0.6837 & 0.9381 & 0.9953 & 0.9999 & 1.0000 \\
\hline 275. & 1.0000 & 1.0000 & 0.9978 & 0.9576 & 0.7261 & 0.3036 & 0.1000 & 0.3036 & 0.7261 & 0.9576 & 0.9978 & 1.0000 & 1.0000 \\
\hline 300. & 1.0000 & 1.0000 & 0.9990 & 0.9716 & 0.7647 & 0.3245 & 0.1000 & 0.3245 & 0.7647 & 0.9716 & 0.9990 & 1.0000 & 1.0000 \\
\hline 325 . & 1.0000 & 1.0000 & 0.9996 & 0.9814 & 0.7997 & 0.3457 & 0.1000 & 0.3457 & 0.7997 & 0.9814 & 0.9996 & 1,0000 & 1.0000 \\
\hline 350. & 1.0000 & 1.0000 & 0.9998 & 0.9881 & 0.8310 & 0.3672 & 0.1000 & 0.3672 & 0.8310 & 0.9881 & 0.9998 & 1.0000 & 1.0000 \\
\hline 375 . & 1.0000 & 1.0000 & 0.9999 & 0.9925 & 0.8587 & 0.3891 & 0.1000 & 0.3891 & 0.8587 & 0.9925 & 0.9999 & 1.0000 & 1.0000 \\
\hline 400 & 1.0000 & 1.0000 & 1.0000 & 0.9955 & 0.8831 & 0.4112 & 0.1000 & 0.4112 & 0.8831 & 0.9955 & 1.0000 & 1.0000 & 1.0000 \\
\hline 425 . & 1.0000 & 1.0000 & 1.0000 & 0.9973 & 0.9043 & 0.4337 & 0.1000 & 0.4337 & 0.9043 & 0.9973 & 1.0000 & 1.0000 & 1.0000 \\
\hline 450 & 1.0000 & 1.0000 & 1.0000 & 0.9984 & 0.9225 & 0.4564 & 0.1000 & 0.4564 & 0.9225 & 0.9984 & 1.0000 & 1.0000 & 1.0000 \\
\hline 475 & 1.0000 & 1.0000 & 1.0000 & 0.9991 & 0.9380 & 0.4793 & 0.1000 & 0.4793 & 0.9380 & 0.9991 & 1.0000 & 1.0000 & 1.0000 \\
\hline 500 & 1.0000 & 1.0000 & 1.0000 & 0.9995 & 0.9510 & 0.5024 & 0.1000 & 0.5024 & 0.9510 & 0.9995 & 1.0000 & 1.0000 & 1.0000 \\
\hline
\end{tabular}




\section{TABL.E A.21 CLINIC}

THE POWER OF THE TEST OF THE UY WOTHESIS TIAT MX $=$ MO WIEN THE SIGNIFICANCE LEVIL IS 0.05

(MX - MO)

\begin{tabular}{|c|c|c|c|c|c|c|c|c|c|c|c|c|c|}
\hline N & -15.00 & -12.50 & -10.00 & -7.50 & -5.00 & -2.50 & 0.00 & 2.50 & 5.00 & 7.50 & 10.00 & 12.50 & 15.00 \\
\hline 25 . & 0.4658 & 0.3454 & 0.2393 & 0.1550 & 0.0958 & 0.0612 & 0.0500 & 0.0612 & 0.0958 & 0.1550 & 0.2393 & 0.3454 & 0.4658 \\
\hline 50 . & 0.7612 & 0.6045 & 0.4287 & 0.2665 & 0.1445 & 0.0730 & 0.0500 & 0.0730 & 0.1445 & 0.2665 & 0.4287 & 0.6045 & 0.7612 \\
\hline 75. & 0.9091 & 0.7841 & 0.5936 & 0.3775 & 0.1956 & 0.0852 & 0.0500 & 0.0852 & 0.1956 & 0.3775 & 0.5936 & 0.7841 & 0.9091 \\
\hline 100. & 0.9696 & 0.8917 & 0.7245 & 0.4830 & 0.2483 & 0.0979 & 0.0500 & 0.0979 & 0.2483 & 0.4830 & 0.7245 & 0.8917 & 0.9696 \\
\hline 125. & 0.9909 & 0.9496 & 0.8214 & 0.5795 & 0.3020 & 0.1112 & 0.0500 & 0.1112 & 0.3020 & 0.5795 & 0.8214 & 0.9496 & 0.9909 \\
\hline 150. & 0.9975 & 0.9781 & 0.8890 & 0.6649 & 0.3561 & 0.1250 & 0.0500 & 0.1250 & 0.3561 & 0.6649 & 0.8890 & 0.9781 & 0.9975 \\
\hline 175. & 0.9994 & 0.9911 & 0.9337 & 0.7382 & 0.4099 & 0.1393 & 0.0500 & 0.1393 & 0.4099 & 0.7382 & 0.9337 & 0.9911 & 0.9994 \\
\hline 200 . & 0.9999 & 0.9966 & 0.9619 & 0.7996 & 0.4630 & 0.1542 & 0.0500 & 0.1542 & 0.4630 & 0.7996 & 0.9619 & 0.9966 & 0.9999 \\
\hline 225. & 1.0000 & 0.9988 & 0.9790 & 0.8497 & 0.5147 & 0.1697 & 0.0500 & 0.1697 & 0.5147 & 0.8497 & 0.9790 & 0.9988 & 1.0000 \\
\hline 250. & 1.0000 & 0.9996 & 0.9889 & 0.8896 & 0.5647 & 0.1857 & 0.0500 & 0.1857 & 0.5647 & 0.8896 & 0.9889 & 0.9996 & 1.0000 \\
\hline 275. & 1.0000 & 0.9999 & 0.9943 & 0.9206 & 0.6125 & 0.2023 & 0.0500 & 0.2023 & 0.6125 & 0.9206 & 0.9943 & 0.9999 & 1.0000 \\
\hline 300. & 1.0000 & 1.0000 & 0.9972 & 0.9441 & 0.6578 & 0.2195 & 0.0500 & 0.2195 & 0.6578 & 0.9441 & 0.9972 & 1.0000 & 1.0000 \\
\hline 325. & 1.0000 & 1.0000 & 0.9987 & 0.9615 & 0.7004 & 0.2373 & 0.0500 & 0.2373 & 0.7004 & 0.9615 & 0.9987 & 1.0000 & 1.0000 \\
\hline 350. & 1.0000 & 1.0000 & 0.9994 & 0.9741 & 0.7399 & 0.2557 & 0.0500 & 0.2557 & 0.7399 & 0.9741 & 0.9994 & 1.0000 & 1.0000 \\
\hline 375. & 1.0000 & 1.0000 & 0.9997 & 0.9830 & 0.7763 & 0.2746 & 0.0500 & 0.2746 & 0.7763 & 0.9830 & 0.9997 & 1.0000 & 1.0000 \\
\hline 400 . & 1.0000 & 1.0000 & 0.9999 & 0.9891 & 0.8094 & 0.2942 & 0.0500 & 0.2942 & 0.8094 & 0.9891 & 0.9999 & 1.0000 & 1.0000 \\
\hline 425. & 1.0000 & 1.0000 & 1.0000 & 0.9932 & 0.8393 & 0.3144 & 0.0500 & 0.3144 & 0.8393 & 0.9932 & 1.0000 & 1.0000 & 1.0000 \\
\hline 450 . & 1.0000 & 1.0000 & 1.0000 & 0.9959 & 0.8659 & 0.3351 & 0.0500 & 0.3351 & 0.8659 & 0.9959 & 1.0000 & 1.0000 & 1.0000 \\
\hline 475. & 1.0000 & 1.0000 & 1.0000 & 0.9976 & 0.8894 & 0.3565 & 0.0500 & 0.3565 & 0.8894 & 0.9976 & 1.0000 & 1.0000 & 1.0000 \\
\hline 500 & 1.0000 & 1.0000 & 1.0000 & 0.9986 & 0.9098 & 0.3784 & 0.0500 & 0.3784 & 0.9098 & 0.9986 & 1.0000 & 1.0000 & 1.0000 \\
\hline
\end{tabular}




\section{TALLE A.22 HOSPITAL}

THE POWER OF THE TEST OF THE HYFOHLSIS THAT MX = MO WHEN THE SIGNIFICANCE LLVEL IS 0.20

$$
\text { (MX - MO) }
$$

\begin{tabular}{|c|c|c|c|c|c|c|c|c|c|c|c|c|c|}
\hline N & -15.00 & -12.50 & -10.00 & -7.50 & -5.00 & -2.50 & 0.00 & 2.50 & 5.00 & 7.50 & 10.00 & 12.50 & 15.00 \\
\hline 7. & 0.2306 & 0.2213 & 0.2136 & 0.2076 & 0.2033 & 0.2007 & 0.1998 & 0.2007 & 0.2033 & 0.2076 & 0.2136 & 0.2213 & 0.2306 \\
\hline 14. & 0.2635 & 0.2445 & 0.2286 & 0.2161 & 0.2071 & 0.2017 & 0.1998 & 0.2017 & 0.2071 & 0.2161 & 0.2286 & 0.2445 & 0.2635 \\
\hline 21. & 0.2987 & 0.2696 & 0.2451 & 0.2256 & 0.2114 & 0.2027 & 0.1998 & 0.2027 & 0.2114 & 0.2256 & 0.2451 & 0.2696 & 0.2987 \\
\hline 28. & 0.3366 & 0.2970 & 0.2632 & 0.2360 & 0.2161 & 0.2034 & 0.1998 & $0.203 y$ & 0.2161 & 0.2360 & 0.2632 & 0.2970 & 0.3366 \\
\hline 35 . & 0.3772 & 0.3269 & 0.2832 & 0.2477 & 0.2214 & 0.2053 & 0.1998 & 0.2053 & 0.2214 & 0.2477 & 0.2832 & 0.3269 & 0.3712 \\
\hline 42. & 0.4209 & 0.3596 & 0.3054 & 0.2607 & 0.2274 & 0.2068 & 0.1998 & 0.2068 & 0.2274 & 0.2607 & 0.3054 & 0.3546 & 0.4209 \\
\hline 49. & 0.4676 & 0.3954 & 0.3302 & 0.2755 & 0.2342 & 0.2086 & 0.1998 & 0.2086 & 0.2342 & 0.2755 & 0.3302 & 0.3954 & 0.4676 \\
\hline 56. & 0.5177 & 0.4348 & 0.3580 & 0.2923 & 0.2421 & 0.2106 & 0.1998 & 0.2106 & 0.2421 & 0.2923 & 0.3580 & 0.4348 & 0.5177 \\
\hline 63. & 0.5712 & 0.4781 & 0.3893 & 0.3116 & 0.2512 & 0.2130 & 0.1998 & 0.2130 & 0.2512 & 0.3116 & 0.3893 & 0.4781 & 0.5712 \\
\hline 70 . & 0.6278 & 0.5259 & 0.4249 & 0.3340 & 0.2620 & 0.2158 & 0.1998 & 0.2158 & 0.2620 & 0.3340 & 0.4249 & 0.5259 & 0.6278 \\
\hline 77. & 0.6872 & 0.5784 & 0.4655 & 0.3603 & 0.2748 & 0.2191 & 0.1998 & 0.2191 & 0.2748 & 0.3603 & 0.4655 & 0.5784 & 0.6872 \\
\hline 84. & 0.7485 & 0.6360 & 0.5121 & 0.3914 & 0.2904 & 0.2233 & 0.1998 & 0.2233 & 0.2904 & 0.3914 & 0.5121 & 0.6360 & 0.7485 \\
\hline 91. & 0.8102 & 0.6987 & 0.5659 & 0.4290 & 0.3097 & .2285 & 0.1998 & 0.2285 & 0.3097 & 0.4290 & 0.3659 & 0.6987 & 0.8102 \\
\hline 98. & 0.8695 & 0.7655 & 0.6281 & 0.4749 & 0.3341 & 0.2353 & 0.1998 & 0.2353 & 0.3341 & 0.4749 & 0.6281 & 0.7655 & 0.8695 \\
\hline 105. & 0.9225 & 0.8345 & 0.6998 & 0.5322 & 0.3662 & 0.2445 & 0.1998 & 0.2445 & 0.3662 & 0.5322 & 0.6998 & 0.8345 & 0.9225 \\
\hline 112. & 0.9642 & 0.9011 & 0.7808 & 0.6048 & 0.4101 & 0.2575 & 0.1998 & 0.2575 & 0.4101 & 0.6048 & 0.7808 & 0.9011 & 0.9642 \\
\hline 119. & 0.9897 & 0.9568 & 0.8677 & 0.6980 & 0.4733 & 0.2774 & 0.1998 & 0.2774 & 0.4733 & 0.6980 & 0.8677 & 0.9568 & 0.9897 \\
\hline 126. & 0.9990 & 0.9907 & 0.9479 & 0.8157 & 0.5712 & 0.3116 & 0.1998 & 0.3116 & 0.5712 & 0.8157 & 0.9479 & 0.9907 & 0.9990 \\
\hline 33. & 1.0000 & 0.9998 & 0.9944 & 0.9433 & 0.7358 & 0.3845 & 0.1998 & 0.3845 & 0.7358 & 0.9433 & 0.9944 & 0.9998 & 1.6000 \\
\hline 40 . & 1.0000 & 1.0000 & 1.0000 & 0.9998 & 0.9755 & 0.6360 & 0.1998 & 0.6360 & 0.9755 & 0.9998 & 1.0000 & 1.0000 & 1.0000 \\
\hline
\end{tabular}


TABLE A.23 HOSPITAL

THE POWLR OF THE TEST OF THE HYPOTHESIS THAT MX = MO WHEN IHE SIGNIHICANCr LEVE, $1 \mathrm{~S}$ 0.10

\begin{tabular}{|c|c|c|c|c|c|c|c|c|c|c|c|c|c|c|}
\hline \multirow[b]{2}{*}{$\mathbf{N}$} & \multicolumn{14}{|c|}{$(M X-M 0)$} \\
\hline & & -15.00 & -12.50 & -10.00 & -7.50 & -5.00 & -2.50 & 0.00 & 2.50 & 5.00 & 7.50 & 10.00 & 12.50 & 15.00 \\
\hline 7. & & 0.1234 & 0.1163 & 0.1104 & 0.1058 & 0.1026 & 0.1006 & 0.1000 & 0.1006 & 0.1026 & 0.1058 & 0.1104 & 0.1163 & 0.1234 \\
\hline 14. & & 0.1492 & 0.1342 & 0.1219 & 0.1123 & 0.1055 & 0.1013 & 0.1000 & 0.1013 & 0.1055 & 0.1123 & 0.1219 & 0.1342 & 0.1492 \\
\hline 21. & & 0.1775 & 0.1541 & 0.1347 & 0.1196 & 0.1087 & 0.1022 & 0.1000 & 0.1022 & 0.1087 & 0.1196 & 0.1347 & 0.1541 & 0.1775 \\
\hline 28 . & & 0.2088 & 0.1761 & 0.1490 & 0.1276 & 0.1123 & 0.1031 & 0.1000 & 0.1031 & 0.1123 & 0.1276 & 0.1490 & 0.1761 & 0.2088 \\
\hline 35 . & & 0.2435 & 0.2007 & 0.1650 & 0.1367 & 0.1164 & 0.1041 & 0.1000 & 0.1041 & 0.1164 & 0.1367 & 0.1650 & 0.2007 & 0.2435 \\
\hline 42. & & 0.2819 & 0.2283 & 0.1830 & 0.1470 & 0.1210 & 0.1052 & 0.1000 & 0.1052 & 0.1210 & 0.1470 & 0.1830 & 0.2283 & 0.2819 \\
\hline 49 . & & 0.3246 & 0.2593 & 0.2035 & 0.1588 & 0.1263 & 0.1066 & 0.1000 & 0.1066 & 0.1263 & 0.1588 & 0.2035 & 0.2593 & 0.3246 \\
\hline 56. & & 0.3721 & 0.2944 & 0.2269 & 0.1723 & 0.1324 & 0.1081 & 0.1000 & 0.1081 & 0.1324 & 0.1723 & 0.2269 & 0.2944 & 0.3721 \\
\hline 63. & & 0.4250 & 0.3344 & 0.2540 & 0.1881 & 0.1395 & 0.1099 & 0.1000 & 0.1099 & 0.1395 & 0.1881 & 0.2540 & 0.3344 & 0.4250 \\
\hline 70. & ' & 0.4838 & 0.3800 & 0.2855 & 0.2067 & 0.1480 & 0.1121 & 0.1000 & 0.1121 & 0.1480 & 0.2067 & 0.2855 & 0.3800 & 0.4838 \\
\hline 77. & & 0.5488 & 0.4324 & 0.3226 & 0.2289 & 0.1582 & 0.1146 & 0.1000 & 0.1146 & 0.1582 & 0.2289 & 0.3226 & 0.4324 & 0.5488 \\
\hline 84. & & 0.6200 & 0.4926 & 0.3667 & 0.2558 & 0.1707 & 0.1178 & 0.1000 & 0.1178 & 0.1707 & 0.2558 & 0.3667 & 0.4926 & 0.6200 \\
\hline 91. & & 0.6966 & 0.5617 & 0.4197 & 0.2892 & 0.1865 & 0.1219 & 0.1000 & 0.1219 & 0.1865 & 0.2892 & 0.4197 & 0.5617 & 0.6966 \\
\hline 98. & & 0.7765 & 0.6406 & 0.4841 & 0.3314 & 0.2068 & 0.1271 & 0.1000 & 0.1271 & 0.2068 & 0.3314 & 0.4841 & 0.6406 & 0.7765 \\
\hline 105. & & 0.8552 & 0.7286 & 0.5630 & 0.3862 & 0.2340 & 0.1342 & 0.1000 & 0.1342 & 0.2340 & 0.3862 & 0.5630 & 0.7286 & 0.8552 \\
\hline 112. & & 0.9248 & 0.8224 & 0.6594 & 0.4595 & 0.2723 & 0.1444 & 0.1000 & 0.1444 & 0.2723 & 0.4595 & 0.6594 & 0.8224 & 0.9248 \\
\hline 119. & & 0.9744 & 0.9117 & 0.7740 & 0.5610 & 0.3299 & 0.1603 & 0.1000 & 0.1603 & 0.3299 & 0.5610 & 0.7740 & 0.9117 & 0.9744 \\
\hline 126. & & 0.9967 & 0.9766 & 0.8965 & 0.7038 & 0.4250 & 0.1881 & 0.1000 & 0.1881 & 0.4250 & 0.7038 & 0.8965 & 0.9766 & 0.9967 \\
\hline 133. & & 1.0000 & 0.9991 & 0.9852 & 0.8888 & 0.6048 & 0.2498 & 0.1000 & 0.2498 & 0.6048 & 0.8888 & 0.9852 & 0.9991 & 1.0000 \\
\hline 140. & & 1.0000 & 1.0000 & 1.0000 & 0.9994 & 0.9458 & 0.4926 & 0.1000 & 0.4926 & 0.9458 & 0.9994 & 1.0000 & 1.0000 & 1.0000 \\
\hline
\end{tabular}


TARLE A. 24 HOSPITAL

THE POHER OF THE TESI OF THE HY HO'HESIS THAT MX $=$ MO WIEN THE SIGNIFICANCE LEVEL IS 0.05

\begin{tabular}{|c|c|c|c|c|c|c|c|c|c|c|c|c|c|}
\hline \multirow[b]{2}{*}{$\mathbf{N}$} & \multicolumn{13}{|c|}{$(M X-M 0)$} \\
\hline & -15.00 & -12.50 & -10.00 & -7.50 & -5.00 & -2.50 & 0.00 & 2.50 & 5.00 & 7.50 & 10.00 & 12.50 & 15.00 \\
\hline 7. & 0.0660 & 0.0611 & 0.0571 & 0.0540 & 0.0518 & 0.0501 & 0.0500 & 0.0504 & 0.0518 & 0.0540 & 0.0571 & 0.0611 & 0.0660 \\
\hline 14. & 0.0841 & 0.0736 & 0.0650 & 0.0584 & 0.0537 & 0.0509 & 0.0500 & 0.0509 & 0.0537 & 0.0584 & 0.0650 & 0.0736 & 0.0841 \\
\hline 21. & 0.1046 & 0.0876 & 0.0739 & 0.0634 & 0.0559 & 0.0515 & 0.0500 & 0.0515 & 0.0559 & 0.0634 & 0.0739 & 0.0876 & 0.1046 \\
\hline 28. & 0.1278 & 0.1036 & 0.0840 & 0.0690 & 0.0584 & 0.0521 & 0.0500 & 0.0521 & 0.0584 & 0.0690 & 0.0840 & 0.1036 & 0.1278 \\
\hline 35 . & 0.1543 & 0.1217 & 0.0954 & 0.0753 & 0.0612 & 0.0528 & 0.0500 & 0.0528 & 0.0612 & 0.0753 & 0.0954 & 0.1217 & 0.1543 \\
\hline 42 . & 0.1847 & 0.1426 & 0.1086 & 0.0826 & 0.0613 & 0.0536 & 0.0500 & 0.0536 & 0.0643 & 0.0826 & 0.1086 & 0.1426 & 0.1847 \\
\hline 49. & 0.2196 & 0.1667 & 0.1238 & 0.0910 & 0.0680 & 0.0545 & 0.0500 & 0.0545 & 0.0680 & 0.0910 & 0.1238 & 0.1667 & 0.2196 \\
\hline 56 . & 0.2599 & 0.1948 & 0.1416 & 0.1008 & 0.0723 & 0.0555 & 0.0500 & 0.0555 & 0.0723 & 0.1008 & 0.1416 & 0.1948 & 0.2599 \\
\hline 63 . & 0.3066 & 0.2277 & 0.1625 & 0.1123 & 0.0773 & 0.0567 & 0.0500 & 0.0567 & 0.0773 & 0.1123 & 0.1625 & 0.2277 & 0.3066 \\
\hline 70 . & 0.3607 & 0.2667 & 0.1876 & 0.1262 & 0.0833 & 0.0582 & 0.0500 & 0.0582 & 0.0833 & 0.1262 & 0.1876 & 0.2667 & 0.3607 \\
\hline 77. & 0.4235 & 0.3132 & 0.2179 & 0.1431 & 0.0906 & 0.0600 & 0.0500 & 0.0600 & 0.0906 & 0.1431 & 0.2179 & 0.3132 & 0.4235 \\
\hline 84 . & 0.4961 & 0.3690 & 0.2552 & 0.1640 & 0.0996 & 0.0622 & 0.0500 & 0.0622 & 0.0996 & 0.1640 & 0.2552 & 0.3690 & 0.4961 \\
\hline 91 . & 0.5791 & 0.4364 & 0.3018 & 0.1905 & 0.1111 & 0.0650 & 0.0500 & 0.0650 & 0.1111 & 0.1905 & 0.3018 & 0.4364 & 0.5791 \\
\hline 98. & 0.6720 & 0.5178 & 0.3610 & 0.2252 & 0.1263 & 0.0686 & 0.0500 & 0.0686 & 0.1263 & 0.2252 & 0.3610 & 0.5178 & 0.6720 \\
\hline 105 . & 0.7716 & 0.6154 & 0.4376 & 0.2721 & 0.1470 & 0.0736 & 0.0500 & 0.0736 & 0.1470 & 0.2721 & 0.4376 & 0.6154 & 0.7716 \\
\hline 112 . & 0.8693 & 0.7289 & 0.5381 & 0.3381 & 0.1770 & 0.0808 & 0.0500 & 0.0808 & 0.1770 & 0.3381 & 0.5381 & 0.7289 & 0.8693 \\
\hline 119. & 0.9490 & 0.8500 & 0.6690 & 0.4357 & 0.2240 & 0.0920 & 0.0500 & 0.0920 & 0.2240 & 0.4357 & 0.6690 & 0.8500 & 0.9490 \\
\hline 126. & 0.9918 & 0.9529 & 0.8282 & 0.5871 & 0.3066 & 0.1123 & 0.0500 & 0.1123 & 0.3066 & 0.5871 & 0.8282 & 0.9529 & 0.9918 \\
\hline 133 . & 0.9999 & 0.9976 & 0.9686 & 0.8173 & 0.4802 & 0.1593 & 0.0500 & 0.1593 & 0.4802 & 0.8173 & 0.9686 & 0.9976 & 0.9999 \\
\hline 0 . & 1.0060 & 1.0000 & 1.0000 & 0.9982 & 0.9015 & 0.3690 & 0.0500 & 0.3690 & 0.9015 & 0.9982 & 1.0000 & 1.0000 & 1.0000 \\
\hline
\end{tabular}


TABLLE A.25 NURSING HOHE

THE POWER OF THE TEST OF TIE HYPOTHESIS THAT MX = MO WIEN TIE SIGNIFICANCE LEVEL IS 0.20

\begin{tabular}{|c|c|c|c|c|c|c|c|c|c|c|c|c|c|}
\hline \multirow[b]{2}{*}{$\mathbf{N}$} & \multicolumn{13}{|c|}{$(M X-M O)$} \\
\hline & -15.00 & -12.50 & -10.00 & -7.50 & -5.00 & -2.50 & 0.00 & 2.50 & 5.00 & 7.50 & 10.00 & 12.50 & 15.00 \\
\hline 1. & 0.2335 & 0.2233 & 0.2149 & 0.2084 & 0.2036 & 0.2008 & 0.1998 & 0.2008 & 0.2036 & 0.2084 & 0.2149 & 0.2233 & 0.2335 \\
\hline 2. & 0.2697 & 0.2489 & 0.2315 & 0.2178 & 0.2079 & 0.2019 & 0.1998 & 0.2019 & 0.2079 & 0.2178 & 0.2315 & 0.2489 & 0.2697 \\
\hline 3. & 0.3088 & 0.2769 & 0.2499 & 0.2283 & 0.2126 & 0.2030 & 0.1998 & 0.2030 & 0.2126 & 0.2283 & 0.2499 & 0.2769 & 0.3088 \\
\hline 4. & 0.3509 & 0.3075 & 0.2702 & 0.2401 & 0.2179 & 0.2044 & 0.1998 & 0.2044 & 0.2179 & 0.2401 & 0.2702 & 0.3075 & 0.3509 \\
\hline 5 . & 0.3964 & 0.3412 & 0.2929 & 0.2533 & 0.2240 & 0.2059 & 0.1998 & 0.2059 & 0.2240 & 0.2533 & 0.2929 & 0.3412 & 0.3964 \\
\hline 6. & 0.4455 & 0.3783 & 0.3183 & 0.2684 & 0.2309 & 0.2077 & 0.1998 & 0.2077 & 0.2309 & 0.2684 & 0.3183 & 0.3783 & 0.4455 \\
\hline 7. & 0.4984 & 0.4194 & 0.3471 & 0.2857 & 0.2390 & 0.2098 & 0.1998 & 0.2098 & 0.2390 & 0.2857 & 0.3471 & 0.4194 & 0.4984 \\
\hline 8. & 0.5551 & 0.4649 & 0.3797 & 0.3057 & 0.2484 & 0.2122 & 0.1998 & 0.2122 & 0.2484 & 0.3057 & 0.3797 & 0.4649 & 0.5551 \\
\hline 9. & 0.6156 & 0.5154 & 0.4170 & 0.3290 & 0.2596 & 0.2151 & 0.1998 & 0.2151 & 0.2596 & 0.3290 & 0.4170 & 0.5154 & 0.6156 \\
\hline 10. & 0.6795 & 0.5714 & 0.4600 & 0.3567 & 0.2730 & 0.2187 & 0.1998 & 0.2187 & 0.2730 & 0.3567 & 0.4600 & 0.5714 & 0.6795 \\
\hline 11. & 0.7457 & 0.6332 & 0.5098 & 0.3899 & 0.2896 & 0.2231 & 0.1998 & 0.2231 & 0.2896 & 0.3899 & 0.5098 & 0.6332 & 0.7457 \\
\hline 12. & 0.8123 & 0.7009 & 0.5679 & 0.4304 & 0.3104 & 0.2288 & 0.1998 & 0.2288 & 0.3104 & 0.4304 & 0.5679 & 0.7009 & 0.8123 \\
\hline 13. & 0.8760 & 0.7734 & 0.6358 & 0.4808 & 0.3374 & 0.2362 & 0.1998 & 0.2362 & 0.3374 & 0.4808 & 0.6358 & 0.7734 & 0.8760 \\
\hline 14. & 0.9316 & 0.8478 & 0.7147 & 0.5448 & 0.3736 & 0.2466 & 0.1998 & 0.2466 & 0.3736 & 0.5448 & 0.7147 & 0.8478 & 0.9316 \\
\hline 15. & 0.9728 & 0.9177 & 0.8040 & 0.6277 & 0.4248 & 0.2620 & 0.1998 & 0.2620 & 0.4248 & 0.6277 & 0.8040 & 0.9177 & 0.9728 \\
\hline 16. & 0.9945 & 0.9715 & 0.8974 & 0.7361 & 0.5022 & $0.28 \%$ & 0.1998 & 0.2870 & 0.5022 & 0.7361 & 0.8974 & 0.9715 & 0.9945 \\
\hline 17. & 0.9998 & 0.9969 & 0.9735 & 0.8711 & 0.6300 & 0.3349 & 0.1998 & 0.3349 & 0.6300 & 0.8711 & 0.9735 & 0.9969 & 0.9998 \\
\hline 18. & 1.0000 & 1.0000 & 0.9997 & 0.9871 & 0.8553 & 0.4627 & 0.1998 & 0.4627 & 0.8553 & 0.9871 & 0.9997 & 1.0000 & 1.0000 \\
\hline
\end{tabular}


TABLE A.26 NUISING HONE

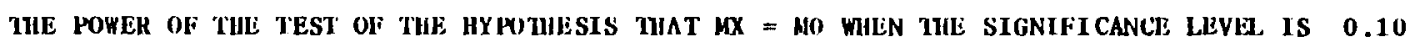

\begin{tabular}{|c|c|c|c|c|c|c|c|c|c|c|c|c|c|}
\hline \multirow[b]{2}{*}{$\mathbf{N}$} & \multicolumn{13}{|c|}{$(M X-M 0)$} \\
\hline & -15.00 & -12.50 & -10.00 & -7.50 & -5.00 & -2.50 & 0.00 & 2.50 & 5.00 & 7.50 & 10.00 & 12.50 & 15.00 \\
\hline 1. & 0.1257 & 0.1179 & 0.1114 & 0.1064 & 0.1028 & 0.1007 & 0.1000 & 0.1007 & 0.1028 & 0.1064 & 0.1114 & 0.1179 & 0.1257 \\
\hline 2. & 0.1541 & 0.1377 & 0.1242 & 0.1136 & 0.1060 & 0.1015 & 0.1000 & 0.1015 & 0.1060 & 0.1136 & 0.1242 & 0.1377 & 0.1541 \\
\hline 3. & 0.1857 & 0.1599 & 0.1385 & 0.1217 & 0.1096 & 0.1024 & 0.1000 & 0.1024 & 0.1096 & 0.1217 & 0.1385 & 0.1599 & 0.1857 \\
\hline 4. & 0.2209 & 0.1847 & 0.1545 & 0.1308 & 0.1137 & 0.1034 & 0.1000 & 0.1034 & 0.1137 & 0.1308 & 0.1545 & 0.1847 & 0.2209 \\
\hline 5. & 0.2602 & 0.2127 & 0.1728 & 0.1412 & 0.1184 & 0.1046 & 0.1000 & 0.1046 & 0.1184 & 0.1412 & 0.1728 & 0.2127 & 0.2602 \\
\hline 6. & 0.3042 & 0.2444 & 0.1936 & 0.1531 & 0.1237 & 0.1059 & 0.1000 & 0.1059 & 0.1237 & 0.1531 & 0.1936 & 0.2444 & 0.3042 \\
\hline 7. & 0.3535 & 0.2806 & 0.2177 & 0.1669 & 0.1299 & 0.1075 & 0.1000 & 0.1075 & 0.1299 & 0.1669 & 0.2177 & 0.2806 & 0.3535 \\
\hline 8 . & 0.4089 & 0.3221 & 0.2456 & 0.1832 & 0.1373 & 0.1093 & 0.1000 & 0.1093 & 0.1373 & 0.1832 & 0.2456 & 0.3221 & 0.4089 \\
\hline 9. & 0.4709 & 0.3699 & 0.2784 & 0.2025 & 0.1461 & 0.1116 & 0.1000 & 0.1116 & 0.1461 & 0.2025 & 0.2784 & 0.3699 & 0.4709 \\
\hline 10. & 0.5401 & 0.4252 & 0.3175 & 0.2258 & 0.1568 & 0.1143 & 0.1000 & 0.1143 & 0.1568 & 0.2258 & 0.3175 & 0.4252 & 0.5401 \\
\hline 11. & 0.6165 & 0.4896 & 0.3644 & 0.2544 & 0.1701 & 0.1177 & 0.1000 & 0.1177 & 0.1701 & 0.2544 & 0.3644 & 0.4896 & 0.6165 \\
\hline 12. & 0.6993 & 0.5643 & 0.4217 & 0.2905 & 0.1871 & 0.1220 & 0.1000 & 0.1220 & 0.1871 & 0.2905 & 0.4217 & 0.5643 & 0.6993 \\
\hline 13. & 0.7857 & 0.6502 & 0.4923 & 0,3369 & 0.2095 & 0.1278 & 0,1000 & 0,1278 & 0.2095 & 0.3369 & 0.4923 & 0.6502 & 0.7857 \\
\hline 14. & 0.8697 & 0.7464 & 0.5801 & 0.3986 & 0.2403 & 0.1359 & 0.1000 & 0.1359 & 0.2403 & 0.3986 & 0.5801 & 0.7464 & 0.8697 \\
\hline 15 . & 0.9407 & 0.8476 & 0.6887 & 0.4837 & 0.2855 & 0.1480 & 0.1000 & 0.1480 & 0.2855 & 0.4837 & 0.6887 & 0.8476 & 0.9407 \\
\hline 16. & 0.9853 & 0.9383 & 0.8169 & 0.6052 & 0.3572 & 0.1680 & 0.1000 & 0.1680 & 0.3572 & 0.6052 & 0.8169 & 0.9383 & 0.9859 \\
\hline 17. & 0.9993 & 0.9913 & 0.9421 & 0.7788 & 0.4861 & 0.2074 & 0.1000 & 0.2074 & 0.4861 & 0.7788 & 0.9421 & 0.9913 & 0.9993 \\
\hline 18. & 1.0000 & 1.0000 & 0.9988 & 0.9690 & 0.7568 & 0.3200 & 0.1000 & 0.3200 & 0.7568 & 0.9690 & 0.9988 & 1.0000 & 1.0000 \\
\hline
\end{tabular}


TAHLE A.27 NURSING HOME

THE POHER OF THE TEST OF TIE HYYUTHESIS THAT MX = MO WIEN THE SIGNIFI CANCE LLVEL IS 0.05

\begin{tabular}{|c|c|c|c|c|c|c|c|c|c|c|c|c|c|}
\hline \multirow[b]{2}{*}{$\mathbf{N}$} & \multicolumn{13}{|c|}{$(M X-M O)$} \\
\hline & -15.00 & -12.50 & -10.00 & -7.50 & -5.00 & -2.50 & 0.00 & 2.50 & 5.00 & 7.50 & 10.00 & 12.50 & 15.00 \\
\hline 1. & 0.0676 & 0.0622 & 0.0578 & 0.0544 & 0.0519 & 0.0505 & 0.0500 & 0.0505 & 0.0519 & 0.0544 & 0.0578 & 0.0622 & 0.0676 \\
\hline 2. & 0.0877 & 0.0760 & 0.0666 & 0.0593 & 0.0541 & 0.0510 & 0.0500 & 0.0510 & 0.0541 & 0.0593 & 0.0666 & 0.0760 & $0.08 \% 7$ \\
\hline 3. & 0.1106 & 0.0918 & 0.0765 & 0.0648 & 0.0566 & 0.0516 & 0.0500 & 0.0516 & 0.0566 & 0.0648 & 0.0765 & 0.0918 & 0.1106 \\
\hline 4. & 0.1370 & 0.1098 & 0.0879 & 0.0712 & 0.0593 & 0.0523 & 0.0500 & 0.0523 & 0.0593 & 0.0712 & 0.0879 & 0.1098 & 0.1370 \\
\hline 5. & 0.1674 & 0.1307 & 0.1011 & 0.0785 & 0.0625 & 0.0531 & 0.0500 & 0.0531 & 0.0625 & 0.0785 & 0.1011 & 0.1307 & 0.1674 \\
\hline 6. & 0.2027 & 0.1551 & 0.1164 & 0.0869 & 0.0662 & 0.0540 & 0.0500 & 0.0540 & 0.0662 & 0.0869 & 0.1164 & 0.1551 & 0.2027 \\
\hline 7. & 0.2439 & 0.1836 & 0.1345 & 0.0969 & 0.0706 & 0.0551 & 0.0500 & 0.0551 & 0.0706 & 0.0969 & 0.1345 & 0.1836 & 0.2439 \\
\hline 8. & 0.2921 & 0.2175 & 0.1560 & 0.1087 & 0.0757 & 0.0564 & 0.0500 & 0.0564 & 0.0757 & 0.1087 & 0.1560 & 0.2175 & 0.2921 \\
\hline 9. & 0.3486 & 0.2579 & 0.1819 & 0.1231 & 0.0819 & 0.0579 & 0.0500 & 0.0579 & 0.0819 & 0.1231 & 0.1819 & 0.2579 & 0.3486 \\
\hline 10. & 0.4149 & 0.3068 & 0.2137 & 0.1407 & 0.0896 & 0.0597 & 0.0500 & 0.0597 & 0.0896 & 0.1407 & 0.2137 & 0.3068 & 0.4149 \\
\hline 11. & 0.4925 & 0.3662 & 0.2533 & 0.1629 & 0.0992 & 0.0621 & 0.0500 & 0.0621 & 0.0992 & 0.1629 & 0.2533 & 0.3662 & 0.4925 \\
\hline 12. & 0.5821 & 0.4389 & 0.3036 & 0.1916 & 0.1116 & 0.0651 & 0.0500 & 0.0651 & 0.1116 & 0.1916 & 0.3036 & 0.4389 & 0.5821 \\
\hline 13. & 0.6831 & 0.5281 & 0.3687 & 0.2299 & 0.1283 & 0.0691 & 0.0500 & 0.0691 & 0.1283 & 0.2299 & 0.3687 & 0.5281 & 0.6831 \\
\hline 14. & 0.7910 & 0.6362 & 0.4549 & 0.2830 & 0.1519 & 0.0747 & 0.0500 & 0.0747 & 0.1519 & 0.2830 & 0.4549 & 0.6362 & 0.7910 \\
\hline 15. & 0.8935 & 0.7615 & 0.5702 & 0.3606 & 0.1876 & 0.0833 & 0.0500 & 0.0833 & 0.1876 & 0.3606 & 0.5702 & 0.7615 & 0.8935 \\
\hline 16. & 0.9687 & 0.8898 & 0.7219 & 0.4807 & 0.2470 & 0.0976 & 0.0500 & 0.0976 & 0.2470 & 0.4807 & 0.7219 & 0.8898 & 0.9687 \\
\hline 17. & 0.9979 & 0.9804 & 0.8957 & 0.6747 & 0.3628 & 0.1268 & 0.0500 & 0.1268 & 0.3628 & 0.6747 & 0.8957 & 0.9804 & 0.9979 \\
\hline 18. & 1.0000 & 1.0000 & 0.9968 & 0.9396 & 0.6484 & 0.2158 & 0.0500 & 0.2158 & 0.6484 & 0.9396 & 0.9968 & 1.0000 & 1.0000 \\
\hline
\end{tabular}


TABLE A.28 STORE

THE POVER OF THE TEST OF TIL IIYPOTHESIS THAT MX = MO WHHN THE SIGNIFICANCE LEVEJ IS 0.20

\begin{tabular}{|c|c|c|c|c|c|c|c|c|c|c|c|c|c|}
\hline \multirow[b]{2}{*}{ N } & \multicolumn{13}{|c|}{$(M X-M O)$} \\
\hline & -15.00 & -12.50 & -10.00 & -7.50 & -5.00 & -2.50 & 0.00 & 2.50 & 5.00 & 7.50 & 10.00 & 12.50 & 15.00 \\
\hline 25 . & 0.8728 & 0.7695 & 0.6319 & 0.4778 & 0.3357 & 0.2358 & 0.1998 & 0.2358 & 0.3357 & 0.4778 & 0.6319 & 0.7695 & 0.8728 \\
\hline 50. & 0.9842 & 0.9427 & 0.8429 & 0.6692 & 0.4527 & 0.2707 & 0.1998 & 0.2707 & 0.4527 & 0.6692 & 0.8429 & 0.9427 & 0.9842 \\
\hline 75. & 0.9983 & 0.9871 & 0.9366 & 0.7955 & 0.5523 & 0.3046 & 0.1998 & 0.3046 & 0.5523 & 0.7955 & 0.9366 & 0.9871 & 0.9983 \\
\hline 100 . & 0.9998 & 0.9973 & 0.9755 & 0.8762 & 0.6361 & 0.3375 & 0.1998 & 0.3375 & 0.6361 & 0.8762 & 0.9755 & 0.9973 & 0.9998 \\
\hline 125. & 1.0000 & 0.9995 & 0.9909 & 0.9265 & 0.7061 & 0.3694 & 0.1998 & 0.3694 & 0.7061 & 0.9265 & 0.9909 & 0.9995 & 1.0000 \\
\hline 150. & 1.0000 & 0.9999 & 0.9967 & 0.9571 & 0.7641 & 0.4002 & 0.1998 & 0.4002 & 0.7641 & 0.9571 & 0.9967 & 0.9999 & 1.0000 \\
\hline 175 . & 1.0000 & 1.0000 & 0.9988 & 0.9753 & 0.8117 & 0.4300 & 0.1998 & 0.4300 & 0.8117 & 0.9753 & 0.9988 & 1.0000 & 1.0000 \\
\hline 200 . & 1.0000 & 1.0000 & 0.9996 & 0.9860 & 0.8505 & 0.4588 & 0.1998 & 0.4588 & 0.8505 & 0.9860 & 0.9996 & 1.0000 & 1.0000 \\
\hline 225 . & 1.0000 & 1.0000 & 0.9999 & 0.9922 & 0.8820 & 0.4865 & 0.1998 & 0.4865 & 0.8820 & 0.9922 & 0.9999 & 1.0000 & 1.0000 \\
\hline 250 . & 1.0000 & 1.0000 & 1.0000 & 0.9957 & 0.9074 & 0.5133 & 0.1998 & 0.5133 & 0.9074 & 0.9957 & 1.0000 & 1.0000 & 1.0000 \\
\hline 275. & 1.0000 & 1.0000 & 1.0000 & 0.9977 & 0.9276 & 0.5391 & 0.1998 & 0.5391 & 0.9276 & 0.9977 & 1.0000 & 1.0000 & 1.0000 \\
\hline 300 . & 1.0000 & 1.0000 & 1.0000 & 0.9987 & 0.9437 & 0.5639 & 0.1998 & 0.5639 & 0.9437 & 0.9987 & 1.0000 & 1.0000 & 1.0000 \\
\hline 32.5 . & 1.0000 & 1.0000 & 1.0000 & 0.9993 & 0.9565 & 0.5877 & 0.1998 & 0.5877 & 0.9565 & 0.9993 & 1.0000 & 1.0000 & 1.0000 \\
\hline 350 . & 1.0000 & 1.0000 & 1.0000 & 0.9996 & 0.9665 & 0.6106 & 0.1998 & 0.6106 & 0.9665 & 0.9996 & 1.0000 & 1.0000 & 1.0000 \\
\hline 375 . & 1.0000 & 1.0000 & 1.0000 & 0.9998 & 0.9744 & 0.6325 & 0.1998 & 0.6325 & 0.9744 & 0.9998 & 1.0000 & 1.0000 & 1.0000 \\
\hline 400 . & 1.0000 & 1.0000 & 1.0000 & 0.9999 & 0.9805 & 0.6535 & 0.1998 & 0.6535 & 0.9805 & 0.9999 & 1.0000 & 1.0000 & 1.0000 \\
\hline 425. & 1.0000 & 1.0000 & 1.0000 & 1.0000 & 0.9852 & 0.6736 & 0.1998 & 0.6736 & 0.9852 & 1.0000 & 1.0000 & 1.0000 & 1.0000 \\
\hline 450 . & 1.0000 & 1.0000 & 1.0000 & 1.0000 & 0.9888 & 0.6928 & 0.1998 & 0.6928 & 0.9888 & 1.0000 & 1.0000 & 1.0000 & 1.0000 \\
\hline 475 . & 1.0000 & 1.0000 & 1.0000 & 1.0000 & 0.9916 & 0.7112 & 0.1998 & 0.7112 & 0.9916 & 1.0000 & 1.0000 & 1.0000 & 1.0000 \\
\hline 500 & 1.0000 & 1.0000 & 1.0000 & 1.0000 & 0.9937 & 0.7287 & 0.1998 & 0.7287 & 0.9937 & 1.0000 & 1.0000 & 1.0000 & 1.0000 \\
\hline
\end{tabular}


TABLE A. 29 STORE

THE POWER OF THE TEST OF THE HYHOTHESIS THAT MX = MO WHEN TIIE SIGNIFICANCE I.EVEL IS 0.10

\begin{tabular}{|c|c|c|c|c|c|c|c|c|c|c|c|c|c|}
\hline \multirow[b]{2}{*}{$\mathbf{N}$} & \multicolumn{13}{|c|}{$(M X-\dot{M} 0)$} \\
\hline & -15.00 & -12.50 & -10.00 & -7.50 & -5.00 & -2.50 & 0.00 & 2.50 & 5.00 & 7.50 & 10.00 & 12.50 & 15.00 \\
\hline 25. & 0.7811 & 0.6454 & 0.4882 & 0.3341 & 0.2081 & 0.1275 & 0.1000 & 0.1275 & 0.2081 & 0.3341 & 0.4882 & 0.6454 & 0.7811 \\
\hline 50. & 0.9630 & 0.8878 & 0.7399 & 0.5286 & 0.3108 & 0.1549 & 0.1000 & 0.1549 & 0.3108 & 0.5286 & 0.7399 & 0.8878 & 0.9630 \\
\hline 75. & 0.9949 & 0.9689 & 0.8777 & 0.6778 & 0.4060 & 0.1823 & 0.1000 & 0.1823 & 0.4060 & 0.6778 & 0.8777 & 0.9689 & 0.9949 \\
\hline 100. & 0.9994 & 0.9922 & 0.9458 & 0.7861 & 0.4926 & 0.2096 & 0.1000 & 0.2096 & 0.4926 & 0.7861 & 0.9458 & 0.9922 & 0.9994 \\
\hline 125. & 0.9999 & 0.9982 & 0.9771 & 0.8615 & 0.5702 & 0.2367 & 0.1000 & 0.2367 & 0.5702 & 0.8615 & 0.9771 & 0.9982 & 0.9999 \\
\hline 150. & 1.0000 & 0.9996 & 0.9907 & 0.9122 & 0.6388 & 0.2635 & 0.1000 & 0.2635 & 0.6388 & 0.9122 & 0.9907 & 0.9996 & 1.0000 \\
\hline 175. & 1.0000 & 0.9999 & 0.9964 & 0.9455 & 0.6986 & 0.2901 & 0.1000 & 0.2901 & 0.6986 & 0.9455 & 0.9964 & 0.9999 & 1.0000 \\
\hline 200. & 1.0000 & 1.0000 & 0.9986 & 0.9668 & 0.7502 & 0.3164 & 0.1000 & 0.3164 & 0.7502 & 0.9668 & 0.9986 & 1.0000 & 1.0000 \\
\hline 225 . & 1.0000 & 1.0000 & 0.9995 & 0.9801 & 0.7944 & 0.3423 & 0.1000 & 0.3423 & 0.7944 & 0.9801 & 0.9995 & 1.0000 & 1.0000 \\
\hline 250. & 1.0000 & 1.0000 & 0.9998 & 0.9882 & 0.8318 & 0.3678 & 0.1000 & 0.3678 & 0.8318 & 0.9882 & 0.9998 & 1.0000 & 1.0000 \\
\hline 275. & 1.0000 & 1.0000 & 0.9999 & 0.9932 & 0.8633 & 0.3930 & 0.1000 & 0.3930 & 0.8633 & 0.9932 & 0.9999 & 1.0000 & 1.0000 \\
\hline 300. & 1.0000 & 1.0000 & 1.0000 & 0.9961 & 0.8895 & 0.4176 & 0.1000 & 0.4176 & 0.8895 & 0.9961 & 1.0000 & 1.0000 & 1.0000 \\
\hline 325. & 1.0000 & 1.0000 & 1.0000 & 0.9978 & 0.9112 & 0.4419 & 0.1000 & 0.4419 & 0.9112 & 0.9978 & 1.0000 & 1.0000 & 1.0000 \\
\hline 350. & 1.0000 & 1.0000 & 1.0000 & 0.9988 & 0.9291 & 0.4656 & 0.1000 & 0.4656 & 0.9291 & 0.9988 & 1.0000 & 1.0000 & 1.0000 \\
\hline 375. & 1.0000 & 1.0000 & 1.0000 & 0.9993 & 0.9436 & 0.4888 & 0.1000 & 0.4888 & 0.9436 & 0.9993 & 1.0000 & 1.0000 & 1.0000 \\
\hline 400 . & 1.0000 & 1.0000 & 1.0000 & 0.9996 & 0.9554 & 0.5115 & 0.1000 & 0.5115 & 0.9554 & 0.9996 & 1.0000 & 1.0000 & 1.0000 \\
\hline 425. & 1.0000 & 1.0000 & 1.0000 & 0.9998 & 0.9650 & 0.5336 & 0.1000 & 0.5336 & 0.9650 & 0.9998 & 1.0000 & 1.0000 & 1.0000 \\
\hline 450. & 1.0000 & 1.0000 & 1.0000 & 0.9999 & 0.9726 & 0.5551 & 0.1000 & 0.5551 & 0.9726 & 0.9999 & 1.0000 & 1.0000 & 1.0000 \\
\hline 475. & 1.0000 & 1.0000 & 1.0000 & 0.9999 & 0.9787 & 0.5761 & 0.1000 & 0.5761 & 0.9787 & 0.9999 & 1.0000 & 1.0000 & 1.0000 \\
\hline 500 . & 1,0000 & 1.0000 & 1.0000 & 1.0000 & 0.9835 & 0.5965 & 0.1000 & 0.5965 & 0.9835 & 1.0000 & 1.0000 & 1.0000 & 1.0000 \\
\hline
\end{tabular}


TABLE ^. 30 STORE

THE POWER OF THE TEST OF THE HYOOTHESIS THAT $\mathrm{KX}=$ MO WIIN THE SIGNIFICANCE LEVLL IS 0.05

\begin{tabular}{|c|c|c|c|c|c|c|c|c|c|c|c|c|c|}
\hline & & & & & & & $M X-M 0)$ & & & & & & \\
\hline $\mathbf{N}$ & -15.00 & -12.50 & -10.00 & -7.50 & -5.00 & -2.50 & 0.00 & 2.50 & 5.00 & 7.50 & 10.00 & 12.50 & 15.00 \\
\hline 25 . & 0.6776 & 0.5230 & 0.3648 & 0.2275 & 0.1273 & 0.0688 & 0.0500 & 0.0688 & 0.1273 & 0.2275 & 0.3648 & 0.5230 & 0.6776 \\
\hline 50. & 0.9294 & 0.8159 & 0.6285 & 0.4037 & 0.2082 & 0.0882 & 0.0500 & 0.0882 & 0.2082 & 0.4037 & 0.6285 & 0.8159 & 0.9294 \\
\hline 75. & 0.9879 & 0.9395 & 0.8019 & 0.5582 & 0.2896 & 0.1081 & 0.0500 & 0.1081 & 0.2896 & 0.5582 & 0.8019 & 0.9395 & 0.9879 \\
\hline 100 & 0.9982 & 0.9823 & 0.9016 & 0.6836 & 0.3691 & 0.1284 & 0.0500 & 0.1284 & 0.3691 & 0.6836 & 0.9016 & 0.9823 & 0.9982 \\
\hline 125. & 0.9998 & 0.9952 & 0.9538 & 0.7799 & 0.4449 & 0.1491 & 0.0500 & 0.1491 & 0.4449 & 0.7799 & 0.9538 & 0.9952 & 0.9998 \\
\hline 150. & 1.0000 & 0.9988 & 0.9793 & 0.8508 & 0.5159 & 0.1700 & 0.0500 & 0.1700 & 0.5159 & 0.8508 & 0.9793 & 0.9988 & 1.0000 \\
\hline 175. & 1.0000 & 0.9997 & 0.9911 & 0.9011 & 0.5813 & 0.1913 & 0.0500 & 0.1913 & 0.5813 & 0.9011 & 0.9911 & 0.9997 & 1.0000 \\
\hline 200 . & 1.0000 & 0.9999 & 0.9963 & 0.9358 & 0.6406 & 0.2128 & 0.0500 & 0.2128 & 0.6406 & 0.9358 & 0.9963 & 0.9999 & 1.0000 \\
\hline 225 . & 1.0000 & 1.0000 & 0.9985 & 0.9591 & 0.6938 & 0.2344 & 0.0500 & 0.2344 & 0.6938 & 0.9591 & 0.9985 & 1.0000 & 1.0000 \\
\hline 250 . & 1.0000 & 1.0000 & 0.9994 & 0.9744 & 0.7410 & 0.2562 & 0.0500 & 0.2562 & 0.7410 & 0.9744 & 0.9994 & 1.0000 & 1.0000 \\
\hline 275 . & 1.0000 & 1.0000 & 0.9998 & 0.9842 & 0.7823 & 0.2780 & 0.0500 & 0.2780 & 0.7823 & 0.9842 & 0.9998 & 1.0000 & 1.0000 \\
\hline 300 & 1.0000 & 1.0000 & 0.9999 & 0.9904 & 0.8183 & 0.2999 & 0.0500 & 0.2999 & 0.8183 & 0.9904 & 0.9999 & 1.0000 & 1.0000 \\
\hline 325. & 1.0000 & 1.0000 & 1.0000 & 0.9943 & 0.8493 & 0.3218 & 0.0500 & 0.3218 & 0.8493 & 0.9943 & 1.0000 & 1.0000 & 1.0000 \\
\hline 350. & 1.0000 & 1.0000 & 1.0000 & 0.9966 & 0.8757 & 0.3437 & 0.0500 & 0.3437 & 0.8757 & 0.9966 & 1.0000 & 1.0000 & 1.0000 \\
\hline 375. & 1.0000 & 1.0000 & 1.0000 & 0.9981 & 0.8981 & 0.3654 & 0.0500 & 0.3654 & 0.8981 & 0.9981 & 1.0000 & 1.0000 & 1.0000 \\
\hline 400 & 1.0000 & 1.0000 & 1.0000 & 0.9989 & 0.9170 & 0.3870 & 0.0500 & 0.3870 & 0.9170 & 0.9989 & 1.0000 & 1.0000 & 1.0000 \\
\hline 425. & 1.0000 & 1.0000 & 1.0000 & 0.9994 & 0.9328 & 0.4085 & 0.0500 & 0.4085 & 0.9328 & 0.9994 & 1.0000 & 1.0000 & 1.0000 \\
\hline 450 & 1.0000 & 1.0000 & 1.0000 & 0.9996 & 0.9458 & 0.4298 & 0.0500 & 0.4298 & 0.9458 & 0.9996 & 1.0000 & 1.0000 & 1.0000 \\
\hline 475 . & 1.0000 & 1.0000 & 1.0000 & 0.9998 & 0.9566 & 0.4508 & 0.0500 & 0.4508 & 0.9566 & 0.9998 & 1.0000 & 1.0000 & 1.0000 \\
\hline 500 . & 1.0000 & 1.0000 & 1.0000 & 0.9999 & 0.9654 & 0.4716 & 0.0500 & 0.4716 & 0.9654 & 0.9999 & 1.0000 & 1.0000 & 1.0000 \\
\hline
\end{tabular}


TAHLE A.31 SHOPPIN; CENTEK

THE hOWER OF THE TEST OF THE HYWTHESIS THAT $\mathrm{MX}=$ MO WIEN THE SIGNIFICANCE LEVEL IS 0.20

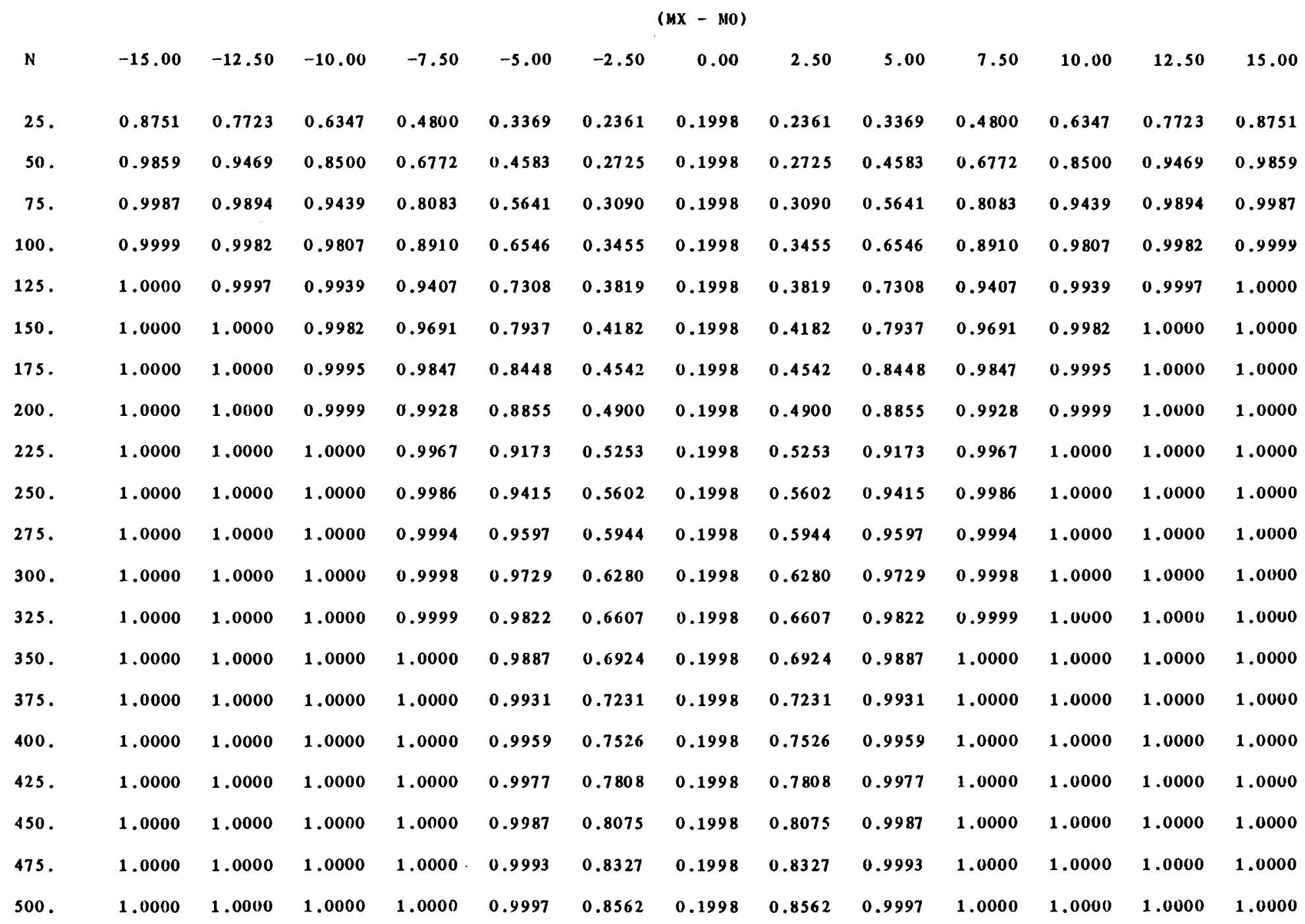




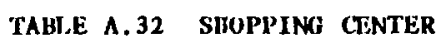

THE POWER OF THE TEST OF THE HYPUTHESIS TIIAT MX = Mo WHEN THE SIGNIF1CANCR LEVHi, IS 0.10

\begin{tabular}{|c|c|c|c|c|c|c|c|c|c|c|c|c|c|}
\hline \multirow[b]{2}{*}{$\mathbf{N}$} & \multicolumn{13}{|c|}{$\left(M X-M_{0}\right)$} \\
\hline & -15.00 & -12.50 & -10.00 & -7.50 & -5.00 & -2.50 & 0.00 & 2.50 & 5.00 & 7.50 & 10.00 & 12.50 & 15.00 \\
\hline 25. & 0.7845 & 0.6489 & 0.4912 & 0.3361 & 0.2091 & 0.1277 & 0.1000 & 0.1277 & 0.2091 & 0.3361 & 0.4912 & 0.6489 & 0.7845 \\
\hline 50. & 0.9665 & 0.8948 & 0.7495 & 0.5376 & 0.3160 & 0.1564 & 0.1000 & 0.1564 & 0.3160 & 0.5376 & 0.7495 & 0.8948 & 0.9665 \\
\hline 75. & 0.9961 & 0.9740 & 0.8897 & 0.6942 & 0.4179 & 0.1859 & 0.1000 & 0.1859 & 0.4179 & 0.6942 & 0.8897 & 0.9740 & 0.9961 \\
\hline 100. & 0.9996 & 0.9945 & 0.9560 & 0.8074 & 0.5127 & 0.2163 & 0.1000 & 0,2163 & 0.5127 & 0.8074 & 0.9560 & 0.9945 & 0.9996 \\
\hline 125. & 1.0000 & 0.9990 & 0.9840 & 0.8844 & 0.5989 & 0.2475 & 0.1000 & 0.2475 & 0.5989 & 0.8844 & 0.9840 & 0.9990 & 1.0000 \\
\hline 150. & 1.0000 & 0.9998 & 0.9947 & $0.933 y$ & 0.6756 & 0.2795 & 0.1000 & 0.2795 & 0.6756 & 0.9339 & 0.9947 & 0.9998 & 1.0000 \\
\hline 175 . & 1.0000 & 1.0000 & 0.9984 & 0.9640 & 0.7425 & 0.3122 & 0.1000 & 0.3122 & 0.7425 & 0.9640 & 0.9984 & 1.0000 & 1.0000 \\
\hline 200. & 1.0000 & 1.0000 & 0.9996 & 0.9813 & 0.7995 & 0.3455 & 0.1000 & 0.3455 & 0.7995 & 0.9813 & 0.9996 & 1.0000 & 1.0000 \\
\hline 225 . & 1.0000 & 1.0000 & 0.9999 & 0.9908 & 0.8470 & 0.3795 & 0.1000 & 0.3795 & 0.8470 & 0.9908 & 0.9999 & 1.0000 & 1.0000 \\
\hline 250 . & 1.0000 & 1.0000 & 1.0000 & 0.9957 & 0.8858 & 0.4139 & 0.1000 & 0.4139 & 0.8858 & 0.9957 & 1.0000 & 1.0000 & 1.0000 \\
\hline 275 . & 1.0000 & 1.0000 & 1.0000 & 0.9981 & 0.9168 & 0.4488 & 0.1000 & 0.4488 & 0.9168 & 0.9981 & 1.0000 & 1.0000 & 1.0000 \\
\hline 300. & 1.0000 & 1.0000 & 1.0000 & 0.9992 & 0.9408 & 0.4839 & 0.1000 & 0.4839 & 0.9408 & 0.9992 & 1.0000 & 1.0000 & 1.0000 \\
\hline 325. & 1.0000 & 1.0000 & $1.0 n 00$ & 0.9997 & 0.9590 & 0.5193 & 0.1000 & 0.5193 & 0.9590 & 0.9997 & 1.0000 & 1.0000 & 1.0000 \\
\hline 350. & 1.0000 & 1.0000 & 1.0000 & 0.9999 & 0.9725 & 0.5546 & 0.1000 & 0.5546 & 0.9725 & 0.9999 & 1.0000 & 1.0000 & 1.0000 \\
\hline 375. & 1.0000 & 1.0000 & 1.0000 & 1.0000 & 0.9821 & 0.5899 & 0.1000 & 0.5899 & 0.9821 & 1.0000 & 1.0000 & 1.0000 & 1.0000 \\
\hline 400 & 1.0000 & 1.0000 & 1.0000 & 1.0000 & 0.9887 & 0.6248 & 0.1000 & 0.6248 & 0.9887 & 1.0000 & 1.0000 & 1.0000 & 1.0000 \\
\hline 425. & 1.0000 & 1.0000 & 1.0000 & 1.0000 & 0.9932 & 0.6593 & 0.1000 & 0.6593 & 0.9932 & 1.0000 & 1.0000 & 1.0000 & 1.0000 \\
\hline 450. & 1.0000 & 1.0000 & 1.0000 & 1.0000 & 0.9960 & 0.6932 & 0.1000 & 0.6932 & 0.9960 & 1.0000 & 1.0000 & 1.0000 & 1.0000 \\
\hline 475. & 1.0000 & 1.0000 & 1.0000 & 1.0000 & 0.9978 & 0.7261 & 0.1000 & 0.7261 & 0.9978 & 1.0000 & 1.0000 & 1.0000 & 1.0000 \\
\hline 500 . & 1.0000 & 1.0000 & 1.0000 & 1.0000 & 0.9988 & 0.7580 & 0.1000 & 0.7580 & 0.9988 & 1.0000 & 1.0000 & 1.0000 & 1.0000 \\
\hline
\end{tabular}


TAMLE $\Lambda .33$ SHOPPING GENTEK

THE FOWEK OF THE TEST OF THE HYPOTHESIS THAT MX = MO WHEN THE SIGNIFICANCE LEVH IS 0.05

\begin{tabular}{|c|c|c|c|c|c|c|c|c|c|c|c|c|c|}
\hline \multirow[b]{2}{*}{$\mathbf{N}$} & \multicolumn{13}{|c|}{$($ HX - MO) } \\
\hline & -15.00 & -12.50 & -10.00 & -7.50 & -5.00 & -2.50 & 0.00 & 2.50 & 5.00 & 7.50 & 10.00 & 12.50 & 15.00 \\
\hline 25. & 0.6816 & 0.5267 & 0.3677 & 0.2292 & 0.1280 & 0.0690 & 0.0500 & 0.0690 & 0.1280 & 0.2292 & 0.3677 & 0.5267 & 0.6816 \\
\hline 50. & 0.9353 & 0.8257 & 0.6398 & 0.4124 & 0.2124 & 0.0893 & 0.0500 & 0.0893 & 0.2124 & 0.4124 & 0.6398 & 0.8257 & 0.9353 \\
\hline 75. & 0.9905 & 0.9482 & 0.8186 & 0.5763 & 0.3001 & 0.1107 & 0.0500 & 0.1107 & 0.3001 & 0.5763 & 0.8186 & 0.9482 & 0.9905 \\
\hline 100. & 0.9989 & 0.9871 & 0.9179 & 0.7100 & 0.3882 & 0.1335 & 0.0500 & 0.1335 & 0.3882 & 0.7100 & 0.9179 & 0.9871 & 0.9989 \\
\hline 125. & 0.9999 & 0.9973 & 0.9664 & 0.8112 & 0.4741 & 0.1575 & 0.0500 & 0.1575 & 0.4741 & 0.8112 & 0.9664 & 0.9973 & 0.9999 \\
\hline 150. & 1.0000 & 0.9995 & 0.9875 & 0.8830 & 0.5558 & 0.1827 & 0.0500 & 0.1827 & 0.5558 & 0.8830 & 0.9875 & 0.9995 & 1.0000 \\
\hline 175. & 1.0000 & 0.9999 & 0.9958 & 0.9311 & 0.6315 & 0.2093 & 0.0500 & 0.2093 & 0.6315 & 0.9311 & 0.9958 & 0.9999 & 1.0000 \\
\hline 200 . & 1.0000 & 1.0000 & 0.9987 & 0.9614 & 0.7001 & 0.2372 & 0.0500 & 0.2372 & 0.7001 & 0.9614 & 0.9987 & 1.0000 & 1.0000 \\
\hline 225 . & 1.0000 & 1.0000 & 0.9996 & 0.9795 & 0.7608 & 0.2663 & 0.0500 & 0.2663 & 0.7608 & 0.9795 & 0.9996 & 1.0000 & 1.0000 \\
\hline 250 . & 1.0000 & 1.0000 & 0.9999 & 0.9897 & 0.8132 & 0.2966 & 0.0500 & 0.2966 & 0.8132 & 0.9897 & 0.9999 & 1.0000 & 1.0000 \\
\hline 275. & 1.0000 & 1.0000 & 1.0000 & 0.9951 & 0.8574 & 0.3282 & 0.0500 & 0.3282 & 0.8574 & 0.9951 & 1.0000 & 1.0000 & 1.0000 \\
\hline 300 . & 1.0000 & 1.0000 & 1.0000 & 0.9978 & 0.8937 & 0.3608 & 0.0500 & 0.3608 & 0.8937 & 0.9978 & 1.0000 & 1.0000 & 1.0000 \\
\hline 325. & 1.0000 & 1.0000 & 1.0000 & 0.9991 & 0.9229 & 0.3946 & 0.0500 & 0.3946 & 0.9229 & 0.9991 & 1.0000 & 1.0000 & 1.0000 \\
\hline 350. & 1.0000 & 1.0000 & 1.0000 & 0.9996 & 0.9456 & 0.4293 & 0.0500 & 0.4293 & 0.9456 & 0.9996 & 1.0000 & 1.0000 & 1.0000 \\
\hline 375. & 1.0000 & 1.0000 & 1.0000 & 0.9999 & 0.9627 & 0.4649 & 0.0500 & 0.4649 & 0.9627 & 0.9999 & 1.0000 & 1.0000 & 1.0000 \\
\hline 400 . & 1.0000 & 1.0000 & 1.0000 & 1.0000 & 0.9753 & 0.5012 & 0.0500 & 0.5012 & 0.9753 & 1.0000 & 1.0000 & 1.0000 & 1.0000 \\
\hline 425. & 1.0000 & 1.0000 & 1.0000 & 1.0000 & 0.9842 & 0.5380 & 0.0500 & 0.5380 & 0.9842 & 1.0000 & 1.0000 & 1.0000 & 1.0000 \\
\hline 450. & 1.0000 & 1.0000 & 1.0000 & 1.0000 & 0.9903 & 0.5752 & 0.0500 & 0.5752 & 0.9903 & 1.0000 & 1.0000 & 1.0000 & 1.0000 \\
\hline 475. & 1.0000 & 1.0000 & 1.0000 & 1.0000 & 0.9943 & 0.6126 & 0.0500 & 0.6126 & 0.9943 & 1.0000 & 1.0000 & 1.0000 & 1.0000 \\
\hline 500. & 1.0000 & 1.0000 & 1.0000 & 1.0000 & 0.9968 & 0.6498 & 0.0500 & 0.6498 & 0.9968 & 1.0000 & 1.0000 & 1.0000 & 1.0000 \\
\hline
\end{tabular}


TAELE A. 34 WAREHOUSE

THE POWEK OF THE TEST OF THE HY ROTHESIS THIA MX = MO WIEN THL SIGNIFICANCE IEVEL IS 0.20

\begin{tabular}{|c|c|c|c|c|c|c|c|c|c|c|c|c|c|}
\hline \multirow[b]{2}{*}{$\mathbf{N}$} & \multicolumn{13}{|c|}{$(M X-M 0)$} \\
\hline & -15.00 & -12.50 & -10.00 & -7.50 & -5.00 & -2.50 & 0.00 & 2.50 & 5.00 & 7.50 & 10.00 & 12.50 & 15.00 \\
\hline 25 . & 0.9947 & 0.9725 & 0.8995 & 0.7390 & 0.5044 & 0.2878 & 0.1998 & 0.2878 & 0.5044 & 0.7390 & 0.8995 & 0.9725 & 0.9947 \\
\hline 50. & 1.0000 & 0.9994 & 0.9905 & 0.9248 & 0.7033 & 0.3680 & 0.1998 & 0.3680 & 0.7033 & 0.9248 & 0.9905 & 0.9994 & 1.0000 \\
\hline 75 . & 1.0000 & 1.0000 & 0.9992 & 0.9800 & 0.8272 & 0.4409 & 0.1998 & 0.4409 & 0.8272 & 0.9800 & 0.9992 & 1.0000 & 1.0000 \\
\hline 100. & 1.0000 & 1.0000 & 0.9999 & 0.9950 & 0.9016 & 0.5068 & 0.1998 & 0.5068 & 0.9016 & 0.9950 & 0.9999 & 1.0000 & 1.0000 \\
\hline 125. & 1.0000 & 1.0000 & 1.0000 & 0.9988 & 0.9450 & 0.5661 & 0.1998 & 0.5661 & 0.9450 & 0.9988 & 1.0000 & 1.0000 & 1.0000 \\
\hline 150. & 1.0000 & 1.0000 & 1.0000 & 0.9997 & 0.9698 & 0.6193 & 0.1998 & 0.6193 & 0.9698 & 0.9997 & 1.0000 & 1.0000 & 1.0000 \\
\hline 175. & 1.0000 & 1.0000 & 1.0000 & 0.9999 & 0.9837 & 0.6669 & 0.1998 & 0.6669 & 0.9837 & 0.9999 & 1.0000 & 1.0000 & 1.0000 \\
\hline 200 & 1.0000 & 1.0000 & 1.0000 & 1.0000 & 0.9913 & 0.7092 & 0.1998 & 0.7092 & 0.9913 & 1.0000 & 1.0000 & 1.0000 & 1.0000 \\
\hline 225 . & 1.0000 & 1.0000 & 1.0000 & 1.0000 & 0.9954 & 0.7468 & 0.1998 & 0.7468 & 0.9954 & 1.0000 & 1.0000 & 1.0000 & 1.0000 \\
\hline 250. & 1.0000 & 1.0000 & 1.0000 & 1.0000 & 0.9976 & 0.7801 & 0.1998 & 0.7801 & 0.9976 & 1.0000 & 1.0000 & 1.0000 & 1.0000 \\
\hline 275. & 1.0000 & 1.0000 & 1.0000 & 1.0000 & 0.9988 & 0.8094 & 0.1998 & 0.8094 & 0.9988 & 1.0000 & 1.0000 & 1.0000 & 1.0000 \\
\hline 300. & 1.0000 & 1.0000 & 1.0000 & 1.0000 & 0.9994 & 0.8352 & 0.1998 & 0.8352 & 0.9994 & 1.0000 & 1.0000 & 1.0000 & 1.0000 \\
\hline 325. & 1.0000 & 1.0000 & 1.0000 & 1.0000 & 0.9997 & 0.8579 & 0.1998 & 0.8579 & 0.9997 & 1.0000 & 1.0000 & 1.0000 & 1.0000 \\
\hline 350. & 1.0000 & 1.0000 & 1.0000 & 1.0000 & 0.9998 & 0.8777 & 0.1998 & 0.8777 & 0.9998 & 1.0000 & 1.0000 & 1.0000 & 1.0000 \\
\hline 375 . & 1.0000 & 1.0000 & 1.0000 & 1.0000 & 0.9999 & 0.8949 & 0.1998 & 0.8949 & 0.9999 & 1.0000 & 1.0000 & 1.0000 & 1.0000 \\
\hline 400 & 1.0000 & 1.0000 & 1.0000 & 1.0000 & 1.0000 & 0.9100 & 0.1998 & 0.9100 & 1.0000 & 1.0000 & 1.0000 & 1.0000 & 1.0000 \\
\hline 425. & 1.0000 & 1.0000 & 1.0000 & 1.0000 & 1.0000 & 0.9230 & 0.1998 & 0.9230 & 1.0000 & 1.0000 & 1.0000 & 1.0000 & 1.0000 \\
\hline 450 . & 1.0000 & 1.0000 & 1.0000 & 1.0000 & 1.0000 & 0.9343 & 0.1998 & 0.9343 & 1.0000 & 1.0000 & 1.0000 & 1.0000 & 1.0000 \\
\hline 475 . & 1.0000 & 1.0000 & 1.0000 & 1.0000 & 1.0000 & 0.9441 & 0.1998 & 0.9441 & 1.0000 & 1.0000 & 1.0000 & 1.0000 & 1.0000 \\
\hline 500 . & 1.0000 & 1.0000 & 1.0000 & 1.0000 & 1.0000 & 0.9525 & 0.1998 & 0.9525 & 1.0000 & 1.0000 & 1.0000 & 1.0000 & 1.0000 \\
\hline
\end{tabular}


TAH.E A.35 WAREHOUSE

TIE POWLik OF THE TEST OF THE HY roTHESIS THAT MX = MO WIIEN THE SIGN1FICANCE LEVEL IS 0.10

\begin{tabular}{|c|c|c|c|c|c|c|c|c|c|c|c|c|c|}
\hline \multirow[b]{2}{*}{$\mathrm{N}$} & \multicolumn{13}{|c|}{$(M X-M O)$} \\
\hline & -15.00 & -12.50 & -10.00 & -7.50 & -5.00 & -2.50 & 0.00 & 2.50 & 5.00 & 7.50 & 10.00 & 12.50 & 15.00 \\
\hline 25 . & 0.9859 & 0.9401 & 0.8200 & 0.6086 & 0.3593 & 0.1686 & 0.1000 & 0.1686 & 0.3593 & 0.6086 & 0.8200 & 0.9401 & 0.9859 \\
\hline 50 . & 0.9999 & 0.9981 & 0.9762 & 0.8588 & 0.5670 & 0.2355 & 0.1000 & 0.2355 & 0.5670 & 0.8588 & 0.9762 & 0.9981 & 0.9999 \\
\hline 75. & 1.0000 & 1.0000 & 0.9975 & 0.9546 & 0.7188 & 0.3000 & 0.1000 & 0.3000 & 0.7188 & 0.9546 & 0.9975 & 1.0000 & 1.0000 \\
\hline 100 . & 1.0000 & 1.0000 & 0.9998 & 0.9866 & 0.8231 & 0.3615 & 0.1000 & 0.3615 & 0.8231 & 0.9866 & 0.9998 & 1.0000 & 1.0000 \\
\hline 125 . & 1.0000 & 1.0000 & 1.0000 & 0.9963 & 0.8917 & 0.4199 & 0.1000 & 0.4199 & 0.8917 & 0.9963 & 1.0000 & 1.0000 & 1.0000 \\
\hline 150. & 1.0000 & 1.0000 & 1.0000 & 0.9990 & 0.9352 & 0.4748 & 0.1000 & 0.4748 & 0.9352 & 0.9990 & 1.0000 & 1.0000 & 1.0000 \\
\hline 175. & 1.0000 & 1.0000 & 1.0000 & 0.9998 & 0.9620 & 0.5261 & 0.1000 & 0.5261 & 0.9620 & 0.9998 & 1.0000 & 1.0000 & 1.0000 \\
\hline 200 . & 1.0000 & 1.0000 & 1.0000 & 0.9999 & 0.9781 & 0.5738 & 0.1000 & 0.5738 & 0.9781 & 0.9999 & 1.0000 & 1.0000 & 1.0000 \\
\hline 225 . & 1.0000 & 1.0000 & 1.0000 & 1.0000 & 0.9876 & 0.6179 & 0.1000 & 0.6179 & 0.9876 & 1.0000 & 1.0000 & 1.0000 & 1.0000 \\
\hline 250 . & 1.0000 & 1.0000 & 1.0000 & 1.0000 & 0.9931 & 0.6585 & 0.1000 & 0.6585 & 0.9931 & 1.0000 & 1.0000 & 1.0000 & 1.0000 \\
\hline 275 . & 1.0000 & 1.0000 & 1.0000 & 1.0000 & 0.9962 & 0.6956 & 0.1000 & 0.6956 & 0.9962 & 1.0000 & 1.0000 & 1.0000 & 1.0000 \\
\hline 300 . & 1.0000 & 1.0000 & 1.0000 & 1.0000 & 0.9979 & 0.7295 & 0.1000 & 0.7295 & 0.9979 & 1.0000 & 1.0000 & 1.0000 & 1.0000 \\
\hline 325 . & 1.0000 & 1.0000 & 1.0000 & 1.0000 & 0.9989 & 0.7603 & 0.1000 & 0.7603 & 0.9989 & 1.0000 & 1.0000 & 1.0000 & 1.0000 \\
\hline 350 . & 1.0000 & 1.0000 & 1.0000 & 1.0000 & 0.9994 & 0.7881 & 0.1000 & 0.7881 & 0.9994 & 1.0000 & 1.0000 & 1.0000 & 1.0000 \\
\hline 375 . & 1.0000 & 1.0000 & 1.0000 & 1.0000 & 0.9997 & 0.8132 & 0.1000 & 0.8132 & 0.9997 & 1.0000 & 1.0000 & 1.0000 & 1.0000 \\
\hline 400 . & 1.0000 & 1.0000 & 1.0000 & 1.0000 & 0.9998 & 0.8358 & 0.1000 & 0.8358 & 0.9998 & 1.0000 & 1.0000 & 1.0000 & 1.0000 \\
\hline 425 . & 1.0000 & 1.0000 & 1.0000 & 1.0000 & 0.9999 & 0.8560 & 0.1000 & 0.8560 & 0.9999 & 1.0000 & 1.0000 & 1.0000 & 1.0000 \\
\hline 450. & 1.0000 & 1.0000 & 1.0000 & 1.0000 & 1.0000 & 0.8740 & 0.1000 & 0.8740 & 1.0000 & 1.0000 & 1.0000 & 1.0000 & 1.0000 \\
\hline 475. & 1.0000 & 1.0000 & 1.0000 & 1.0000 & 1.0000 & 0.8900 & 0.1000 & 0.8900 & 1.0000 & 1.0000 & 1.0000 & 1.0000 & 1.0000 \\
\hline 500. & 1.0000 & 1.0000 & 1.0000 & 1.0000 & 1.0000 & 0.9042 & 0.1000 & 0.9042 & 1.0000 & 1.0000 & 1.0000 & 1.0000 & 1.0000 \\
\hline
\end{tabular}


TABLE A.36 WAREHOUSE

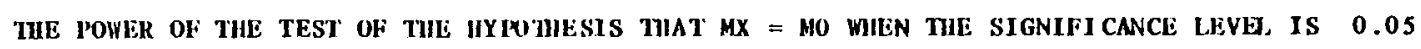

\begin{tabular}{|c|c|c|c|c|c|c|c|c|c|c|c|c|c|}
\hline $\mathbf{N}$ & -15.00 & -12.50 & -10.00 & -7.50 & -5.00 & -2.50 & 0.00 & 2.50 & 5.00 & 7.50 & 10.00 & 12.50 & 15.00 \\
\hline 25. & 0.9700 & 0.8926 & 0.7258 & 0.4842 & 0.2489 & 0.0981 & 0.0500 & 0.0981 & 0.2489 & 0.4842 & 0.7258 & 0.8926 & 0.9700 \\
\hline 50 . & 0.9997 & 0.9950 & 0.9522 & 0.7763 & 0.4417 & 0.1482 & 0.0500 & 0.1482 & 0.4417 & 0.7763 & 0.9522 & 0.9950 & 0.9998 \\
\hline 75 . & 1.0000 & 0.9998 & 0.9936 & 0.9156 & 0.6042 & 0.1993 & 0.0500 & 0.1993 & 0.6042 & 0.9156 & 0.9936 & 0.9998 & 1.0000 \\
\hline 100 & 1.0000 & 1.0000 & 0.9993 & 0.9712 & 0.7298 & 0.2508 & 0.0500 & 0.2508 & 0.7298 & 0.9712 & 0.9993 & 1.0000 & 1.0000 \\
\hline 125. & 1.0000 & 1.0000 & 0.9999 & 0.9909 & 0.8213 & 0.3019 & 0.0500 & 0.3019 & 0.8213 & 0.9909 & 0.9999 & 1.0000 & 1.0000 \\
\hline 150. & 1.0000 & 1.0000 & 1.0000 & 0.9973 & 0.8850 & 0.3522 & 0.0500 & 0.3522 & 0.8850 & 0.9973 & 1.0000 & 1.0000 & 1.0000 \\
\hline 175. & 1.0000 & 1.0000 & 1.0000 & 0.9992 & 0.9277 & 0.4012 & 0.0500 & 0.4012 & 0.9277 & 0.9992 & 1.0000 & 1.0000 & 1.0000 \\
\hline 200 & 1.0000 & 1.0000 & 1.0000 & 0.9998 & 0.9555 & 0.4485 & 0.0500 & 0.4485 & 0.9555 & 0.9998 & 1.0000 & 1.0000 & 1.0000 \\
\hline 225 . & 1.0000 & 1.0000 & 1.0000 & 0.9999 & 0.9731 & 0.4939 & 0.0500 & 0.4939 & 0.9731 & 0.9999 & 1.0000 & 1.0000 & 1.0000 \\
\hline 250. & 1.0000 & 1.0000 & 1.0000 & 1.0000 & 0.9841 & 0.5371 & 0.0500 & 0.5371 & 0.9841 & 1.0000 & 1.0000 & 1.0000 & 1.0000 \\
\hline 275. & 1.0000 & 1.0000 & 1.0000 & 1.0000 & 0.9907 & 0.5780 & 0.0500 & 0.5780 & 0.9907 & 1.0000 & 1.0000 & 1.0000 & 1.0000 \\
\hline 300 . & 1.0000 & 1.0000 & 1.0000 & 1.0000 & 0.9947 & 0.6165 & 0.0500 & 0.6165 & 0.9947 & 1.0000 & 1.0000 & 1.0000 & 1.0000 \\
\hline 325. & 1.0000 & 1.0000 & 1.0000 & 1.0000 & 0.9970 & 0.6525 & 0.0500 & 0.6525 & 0.9970 & 1.0000 & 1.0000 & 1.0000 & 1.0000 \\
\hline 350. & 1.0000 & 1.0000 & 1.0000 & 1.0000 & 0.9983 & 0.6861 & 0.0500 & 0.6861 & 0.9983 & 1.0000 & 1.0000 & 1.0000 & 1.0000 \\
\hline 375. & 1.0000 & 1.0000 & 1.0000 & 1.0000 & 0.9991 & 0.7173 & 0.0500 & 0.7173 & 0.9991 & 1.0000 & 1.0000 & 1.0000 & 1.0000 \\
\hline 400. & 1.0000 & 1.0000 & 1.0000 & 1,0000 & 0.9995 & 0.7461 & 0.0500 & 0.7461 & 0.9995 & 1.0000 & 1.0000 & 1.0000 & 1.0000 \\
\hline 425. & 1.0000 & 1.0000 & 1.0000 & 1.0000 & 0.9997 & 0.7726 & 0.0500 & 0.7726 & 0.9997 & 1.0000 & 1.0000 & 1.0000 & 1.0000 \\
\hline 450 & 1.0000 & 1.0000 & 1.0000 & 1.0000 & 0.9999 & 0.7969 & 0.0500 & 0.7969 & 0.9999 & 1.0000 & 1.0000 & 1.0000 & 1.0000 \\
\hline 475. & 1.0000 & 1.0000 & 1.0000 & 1.0000 & 0.9999 & 0.8190 & 0.0500 & 0.8190 & 0.9999 & 1.0000 & 1.0000 & 1.0000 & 1.0000 \\
\hline 500 . & 1.0000 & 1.0000 & 1.0000 & 1.0000 & 1.0000 & 0.8392 & 0.0500 & 0.8392 & 1.0000 & 1.0000 & 1.0000 & 1.0000 & 1.0000 \\
\hline
\end{tabular}


TABLE A.37 HOTE,/MOTEL

1HE HOWER OF TIIR TEST OF THE HX HOZHESIS THAT MX = HO WHEN MIIE SIGNIFI CANCE LEVEL IS 0.20

\begin{tabular}{|c|c|c|c|c|c|c|c|c|c|c|c|c|c|}
\hline \multirow[b]{2}{*}{$\mathbf{N}$} & \multicolumn{13}{|c|}{$(M X-M O)$} \\
\hline & -15.00 & -12.50 & -10.00 & -7.50 & -5.00 & -2.50 & 0.00 & 2.50 & 5.00 & 7.50 & 10.00 & 12.50 & 15.00 \\
\hline 25. & 0.9992 & 0.9923 & 0.9537 & 0.8267 & 0.5820 & 0.3157 & 0.1998 & 0.3157 & 0.5820 & 0.8267 & 0.9537 & 0.9923 & 0.9992 \\
\hline 50. & 1.0000 & 1.0000 & 0.9987 & 0.9741 & 0.8079 & 0.4274 & 0.1998 & 0.4274 & 0.8079 & 0.9741 & 0.9987 & 1.0000 & 1.0000 \\
\hline 75. & 1.0000 & 1.0000 & 1.0000 & 0.9973 & 0.9232 & 0.5330 & 0.1998 & 0.5330 & 0.9232 & 0.9973 & 1.0000 & 1.0000 & 1.0000 \\
\hline 100. & 1.0000 & 1.0000 & 1.0000 & 0.9998 & 0.9738 & 0.6307 & 0.1998 & 0.6307 & 0.9738 & 0.9998 & 1.0000 & 1.0000 & 1.0000 \\
\hline 125. & 1.0000 & 1.0000 & 1.0000 & 1.0000 & 0.9925 & 0.7185 & 0.1998 & 0.7185 & 0.9925 & 1.0000 & 1.0000 & 1.0000 & 1.0000 \\
\hline 150. & 1.0000 & 1.0000 & 1.0000 & 1.0000 & 0.9983 & 0.7948 & 0.1998 & 0.7948 & 0.9983 & 1.0000 & 1.0000 & 1.0000 & 1.0000 \\
\hline 175. & 1.0000 & 1.0000 & 1.0000 & 1.0000 & 0.9997 & 0.8585 & 0.1998 & 0.8585 & 0.9997 & 1.0000 & 1.0000 & 1.0000 & 1.0000 \\
\hline 200 . & 1.0000 & 1.0000 & 1.0000 & 1.0000 & 1.0000 & 0.9090 & 0.1998 & 0.9090 & 1.0000 & 1.0000 & 1.0000 & 1.0000 & 1.0000 \\
\hline 225 . & 1.0000 & 1.0000 & 1.0000 & 1.0000 & 1.0000 & 0.9464 & 0.1998 & 0.9464 & 1.0000 & 1.0000 & 1.0000 & 1.0000 & 1.0000 \\
\hline 250. & 1.0000 & 1.0000 & 1.0000 & 1.0000 & 1.0000 & 0.9719 & 0.1998 & 0.9719 & 1.0000 & 1.0000 & 1.0000 & 1.0000 & 1.0000 \\
\hline 275. & 1.0000 & 1.0000 & 1.0000 & 1.0000 & 1.0000 & 0.9873 & 0.1998 & 0.9873 & 1.0000 & 1.0000 & 1.0000 & 1.0000 & 1.0000 \\
\hline 300. & 1.0000 & 1.0000 & 1.0000 & 1.0000 & 1.0000 & 0.9954 & 0.1998 & 0.9954 & 1.0000 & 1.0000 & 1.0000 & 1.0000 & 1.0000 \\
\hline 325 . & 1.0000 & 1.0000 & 1.0000 & 1.0000 & 1.0000 & 0.9987 & 0.1998 & 0.9987 & 1.0000 & 1.0000 & 1.0000 & 1.0000 & 1.0000 \\
\hline 350 . & 1.0000 & 1.0000 & 1.0000 & 1.0000 & 1.0000 & 0.9998 & 0.1998 & 0.9998 & 1.0000 & 1.0000 & 1.0000 & 1.0000 & 1.0000 \\
\hline 375 . & 1.0000 & 1.0000 & 1.0000 & 1.0000 & 1.0000 & 1.0000 & 0.1998 & 1.0000 & 1.0000 & 1.0000 & 1.0000 & 1.0000 & 1.0000 \\
\hline 400 & 1.0000 & 1.0000 & 1.0000 & 1.0000 & 1.0000 & 1.0000 & 0.1998 & 1.0000 & 1.0000 & 1.0000 & 1.0000 & 1.0000 & 1.0000 \\
\hline 425 . & 1.0000 & 1.0000 & 1.0000 & 1.0000 & 1.0000 & 1.0000 & 0.1998 & 1.0000 & 1.0000 & 1.0000 & 1.0000 & 1.0000 & 1.0000 \\
\hline 450. & 1.0000 & 1.0000 & 1.0000 & 1.0000 & 1.0000 & 1.0000 & 0.1998 & 1.0000 & 1.0000 & 1.0000 & 1.0000 & 1.0000 & 1.0000 \\
\hline 475 . & 1.0000 & 1.0000 & 1.0000 & 1.0000 & 1.0000 & 1.0000 & 0.1998 & 1.0000 & 1.0000 & 1.0000 & 1.0000 & 1.0000 & 1.0000 \\
\hline 500. & 1.0000 & 1.0000 & 1.0000 & 1.0000 & 1.0000 & 1.0000 & 0.1998 & 1.0000 & 1.0000 & 1.0000 & 1.0000 & 1.0000 & 1.0000 \\
\hline
\end{tabular}


TAMLE A.38 HOTEL/MOTHL

THE POWER OF THE TEST OF TIL HYPO'WHSIS THAT MX = MO WIEN THE SIGNIFICANCE LEVHL IS 0.10

\begin{tabular}{|c|c|c|c|c|c|c|c|c|c|c|c|c|c|}
\hline \multirow[b]{2}{*}{$\mathbf{N}$} & \multicolumn{13}{|c|}{$(M X-M 0)$} \\
\hline & -15.00 & -12.50 & -10.00 & -7.50 & -5.00 & -2.50 & 0.00 & 2.50 & 5.00 & 7.50 & 10.00 & 12.50 & 15.00 \\
\hline 25. & 0.9974 & 0.9803 & 0.9063 & 0.7182 & 0.4360 & 0.1915 & 0.1000 & 0.1915 & 0.4360 & 0.7182 & 0.9063 & 0.9803 & 0.9974 \\
\hline 50. & 1.0000 & 0.9999 & 0.9961 & 0.9431 & 0.6937 & 0.2878 & 0.1000 & 0.2878 & 0.6937 & 0.9431 & 0.9961 & 0.9999 & 1.0000 \\
\hline 75. & 1.0000 & 1.0000 & 0.9999 & 0.9922 & 0.8563 & 0.3870 & 0.1000 & 0.3870 & 0.8563 & 0.9922 & 0.9999 & 1.0000 & 1.0000 \\
\hline 100. & 1.0000 & 1.0000 & 1.0000 & 0.9993 & 0.9425 & 0.4868 & 0.1000 & 0.4868 & 0.9425 & 0.9993 & 1.0000 & 1.0000 & 1.0000 \\
\hline 125. & 1.0000 & 1.0000 & 1.0000 & 1.0000 & 0.9808 & 0.5845 & 0.1000 & 0.5845 & 0.9808 & 1.0000 & 1.0000 & 1.0000 & 1.0000 \\
\hline 150. & 1.0000 & 1.0000 & 1.0000 & 1.0000 & 0.9948 & 0.6770 & 0.1000 & 0.6770 & 0.9948 & 1.0000 & 1.0000 & 1.0000 & 1.0000 \\
\hline 175. & 1.0000 & 1.0000 & 1.0000 & 1.0000 & 0.9989 & 0.7612 & 0.1000 & 0.7612 & 0.9989 & 1.0000 & 1.0000 & 1.0000 & 1.0000 \\
\hline 200 . & 1.0000 & 1.0000 & 1.0000 & 1.0000 & 0.9998 & 0.8343 & 0.1000 & 0.8343 & 0.9998 & 1.0000 & 1.0000 & 1.0000 & 1.0000 \\
\hline 225 . & 1.0000 & 1.0000 & 1.0000 & 1.0000 & 1.0000 & 0.8940 & 0.1000 & 0.8940 & 1.0000 & 1.0000 & 1.0000 & 1.0000 & 1.0000 \\
\hline 250. & 1.0000 & 1.0000 & 1.0000 & 1.0000 & 1.0000 & 0.9390 & 0.1000 & 0.9390 & 1.0000 & 1.0000 & 1.0000 & 1.0000 & 1.0000 \\
\hline 275 . & 1.0000 & 1.0000 & 1.0000 & 1.0000 & 1.0000 & 0.9695 & 0.1000 & 0.9695 & 1.0000 & 1.0000 & 1.0000 & 1.0000 & 1.0000 \\
\hline 300. & 1.0000 & 1. .0000 & 1.0000 & 1.0000 & 1.0000 & 0.9874 & 0.1000 & 0.9874 & 1.0000 & 1.0000 & 1.0000 & 1.0000 & 1.0000 \\
\hline 325 . & 1.0000 & 1.0000 & 1.0000 & 1.0000 & 1.0000 & 0.9961 & 0.1000 & 0.9961 & 1.0000 & 1.0000 & 1.0000 & 1.0000 & 1.0000 \\
\hline 350. & 1.0000 & 1.0000 & 1.0000 & 1.0000 & 1.0000 & 0.9992 & 0.1000 & 0.9992 & 1.0000 & 1.0000 & 1.0000 & 1.0000 & 1.0000 \\
\hline 375. & 1.0000 & 1.0000 & 1.0000 & 1.0000 & 1.0000 & 0.9999 & 0.1000 & 0.9999 & 1.0000 & 1.0000 & 1.0000 & 1.0000 & 1.0000 \\
\hline 400 & 1.0000 & 1.0000 & 1.0000 & 1.0000 & 1.0000 & 1.0000 & 0.1000 & 1.0000 & 1.0000 & 1.0000 & 1.0000 & 1.0000 & 1.0000 \\
\hline 425. & 1.0000 & 1.0000 & 1.0000 & 1.0000 & 1.0000 & 1.0000 & 0.1000 & 1.0000 & 1.0000 & 1.0000 & 1.0000 & 1.0000 & 1.0000 \\
\hline 450. & 1.0000 & 1.0000 & 1.0000 & 1.0000 & 1.0000 & 1.0000 & 0.1000 & 1.0000 & 1.0000 & 1.0000 & 1.0000 & 1.0000 & 1.0000 \\
\hline 475. & 1.0000 & 1.0000 & 1.0000 & 1.0000 & 1.0000 & 1.0000 & 0.1000 & 1.0000 & 1.0000 & 1.0000 & 1.0000 & 1.0000 & 1.0000 \\
\hline 500 & 1.0000 & 1.0000 & 1.0000 & 1.0000 & 1.0000 & 1.0000 & 0.1000 & 1.0000 & 1.0000 & 1.0000 & 1.0000 & 1.0000 & 1.0000 \\
\hline
\end{tabular}


TABLE A.39 NOTLL/MOTLL,

TIE POYER OF TUE TEST OI THE HY POMHESIS THAT MX = MO WHEN IHE SIGNIFI CANCE LEVEL IS 0.05

\begin{tabular}{|c|c|c|c|c|c|c|c|c|c|c|c|c|c|}
\hline \multirow[b]{2}{*}{$\mathbf{N}$} & \multicolumn{13}{|c|}{$(M X-M O)$} \\
\hline & -15.00 & -12.50 & -10.00 & -7.50 & -5.00 & -2.50 & 0.00 & 2.50 & 5.00 & 7.50 & 10.00 & 12.50 & 15.00 \\
\hline 25. & 0.9935 & 0.9594 & 0.8421 & 0.6035 & 0.3165 & 0.1148 & 0.0500 & 0.1148 & 0.3165 & 0.6035 & 0.8421 & 0.9594 & 0.9935 \\
\hline 50 . & 1.0000 & 0.9997 & 0.9904 & 0.8974 & 0.5758 & 0.1894 & 0.0500 & 0.1894 & 0.5758 & 0.8974 & 0.9904 & 0.9997 & 1.0000 \\
\hline 75. & 1.0000 & 1.0000 & 0.9997 & 0.9823 & 0.7730 & 0.2728 & 0.0500 & 0.2728 & 0.7730 & 0.9823 & 0.9997 & 1.0000 & 1.0000 \\
\hline 100 & 1.0000 & 1.0000 & 1.0000 & 0.9980 & 0.8964 & 0.3636 & 0.0500 & 0.3636 & 0.8964 & 0.9980 & 1.0000 & 1.0000 & 1.0000 \\
\hline 125. & 1.0000 & 1.0000 & 1.0000 & 0.9998 & 0.9604 & 0.4594 & 0.0500 & 0.4594 & 0.9604 & 0.9998 & 1.0000 & 1.0000 & 1.0000 \\
\hline 150. & 1.0000 & 1.0000 & 1.0000 & 1.0000 & 0.9877 & 0.5573 & 0.0500 & 0.5573 & 0.9877 & 1.0000 & 1.0000 & 1.0000 & 1.0000 \\
\hline 175. & 1.0000 & 1.0000 & 1.0000 & 1.0000 & 0.9970 & 0.6536 & 0.0500 & 0.6536 & 0.9970 & 1.0000 & 1.0000 & 1.0000 & 1.0000 \\
\hline 200 & 1.0000 & 1.0000 & 1.0000 & 1.0000 & 0.9995 & 0.7442 & 0.0500 & 0.7442 & 0.9995 & 1.0000 & 1.0000 & 1.0000 & 1.0000 \\
\hline 225 . & 1.0000 & 1.0000 & 1.0000 & 1.0000 & 0.9999 & 0.8246 & 0.0500 & 0.8246 & 0.9999 & 1.0000 & 1.0000 & 1.0000 & 1.0000 \\
\hline 250 . & 1.0000 & 1.0000 & 1.0000 & 1.0000 & 1.0000 & 0.8909 & 0.0500 & 0.8909 & 1.0000 & 1.0000 & 1.0000 & 1.0000 & 1.0000 \\
\hline 275. & 1.0000 & 1.0000 & 1.0000 & 1.0000 & 1.0000 & 0.9404 & 0.0500 & 0.9404 & 1.0000 & 1.0000 & 1.0000 & 1.0000 & 1.0000 \\
\hline 300. & 1.0000 & 1.0000 & 1.0000 & 1.0000 & 1.0000 & 0.9728 & 0.0500 & 0.9728 & 1.0000 & 1.0000 & 1.0000 & 1.0000 & 1.0000 \\
\hline 325. & 1.0000 & 1.0000 & 1.0000 & 1.0000 & 1.0000 & 0.9904 & 0.0500 & 0.9904 & 1.0000 & 1.0000 & 1.0000 & 1.0000 & 1.0000 \\
\hline 350. & 1.0000 & 1.0000 & 1.0000 & 1.0000 & 1.0000 & 0.9977 & 0.0500 & 0.9977 & 1.0000 & 1.0000 & 1.0000 & 1.0000 & 1.0000 \\
\hline 375. & 1.0000 & 1.0000 & 1.0000 & 1.0000 & 1.0000 & 0.9997 & 0.0500 & 0.9997 & 1.0000 & 1.0000 & 1.0000 & 1.0000 & 1.0000 \\
\hline 400 . & 1.0000 & 1.0000 & 1.0000 & 1.0000 & 1.0000 & 1.0000 & 0.0500 & 1.0000 & 1.0000 & 1.0000 & 1.0000 & 1.0000 & 1.0000 \\
\hline 425. & 1.0000 & 1.0000 & 1.0000 & 1.0000 & 1.0000 & 1.0000 & 0.0500 & 1.0000 & 1.0000 & 1.0000 & 1.0000 & 1.0000 & 1.0000 \\
\hline 450. & 1.0000 & 1.0000 & 1.0000 & 1.0000 & 1.0000 & 1.0000 & 0.0500 & 1.0000 & 1.0000 & 1.0000 & 1.0000 & 1.0000 & 1.0000 \\
\hline 475. & 1.0000 & 1.0000 & 1.0000 & 1.0000 & 1.0000 & 1.0000 & 0.0500 & 1.0000 & 1.0000 & 1.0000 & 1.0000 & 1.0000 & 1.0000 \\
\hline 500 & 1.0000 & 1.0000 & 1.0000 & 1.0000 & 1.0000 & 1.0000 & 0.0500 & 1.0000 & 1.0000 & 1.0000 & 1.0000 & 1.0000 & 1.0000 \\
\hline
\end{tabular}


TABLE A.40 THEATER/AUHITORI UA

THE POWER OF THE TEST OF TIIE HYPOTHESIS THAT MX = MO WILN THE SIGNIFICANCE LEVIA. IS 0.20

\begin{tabular}{|c|c|c|c|c|c|c|c|c|c|c|c|c|c|}
\hline \multirow[b]{2}{*}{$\mathbf{N}$} & \multicolumn{13}{|c|}{$(M X-M O)$} \\
\hline & -15.00 & -12.50 & -10.00 & -7.50 & -5.00 & -2.50 & 0.00 & 2.50 & 5.00 & 7.50 & 10.00 & 12.50 & 15.00 \\
\hline 8. & 0.7224 & 0.6110 & 0.4915 & 0.3775 & 0.2834 & 0.2214 & 0.1998 & 0.2214 & 0.2834 & 0.3775 & 0.4915 & 0.6110 & 0.7224 \\
\hline 16. & 0.9232 & 0.8355 & 0.7008 & 0.5330 & 0.3667 & 0.2446 & 0.1998 & 0.2446 & 0.3667 & 0.5330 & 0.7008 & 0.8355 & 0.9232 \\
\hline 24. & 0.9830 & 0.9398 & 0.8382 & 0.6639 & 0.4491 & 0.2695 & 0.1998 & 0.2695 & 0.4491 & 0.6639 & 0.8382 & 0.9398 & 0.9830 \\
\hline 32. & 0.9970 & 0.9813 & 0.9205 & 0.7695 & 0.5294 & 0.2964 & 0.1998 & 0.2964 & 0.5294 & 0.7695 & 0.9205 & 0.9813 & 0.9970 \\
\hline 40 . & 0.9996 & 0.9951 & 0.9650 & 0.8505 & 0.6067 & 0.3254 & 0.1998 & 0.3254 & 0.6067 & 0.8505 & 0.9650 & 0.9951 & 0.9996 \\
\hline 48. & 1.0000 & 0.9990 & 0.9864 & 0.9094 & 0.6798 & 0.3568 & 0.1998 & 0.3568 & 0.6798 & 0.9094 & 0.9864 & 0.9990 & 1.0000 \\
\hline 56. & 1.0000 & 0.9998 & 0.9955 & 0.9493 & 0.7474 & 0.3908 & 0.1998 & 0.3908 & 0.7474 & 0.9493 & 0.9955 & 0.9998 & 1.0000 \\
\hline 64. & 1.0000 & 1.0000 & 0.9987 & 0.9742 & 0.8083 & 0.4277 & 0.1998 & 0.4277 & 0.8083 & 0.9742 & 0.9987 & 1.0000 & 1.0000 \\
\hline 72. & 1.0000 & 1.0000 & 0.9997 & 0.9884 & 0.8613 & 0.4677 & 0.1998 & 0.4677 & 0.8613 & 0.9884 & 0.9997 & 1.0000 & 1.0000 \\
\hline 80 . & 1.0000 & 1.0000 & 1.0000 & 0.9955 & 0.9056 & 0.5113 & 0.1998 & 0.5113 & 0.9056 & 0.9955 & 1.0000 & 1.0000 & 1.0000 \\
\hline 88. & 1.0000 & 1.0000 & 1.0000 & 0.9986 & 0.9405 & 0.5585 & 0.1998 & 0.5585 & 0.9405 & 0.9986 & 1.0000 & 1.0000 & 1.0000 \\
\hline 96 . & 1.0000 & 1.0000 & 1.0000 & 0.9996 & 0.9661 & 0.6096 & 0.1998 & 0.6096 & 0.9661 & 0.9996 & 1.0000 & 1.0000 & 1.0000 \\
\hline 104. & 1.0000 & 1.0000 & 1.0000 & u.9999 & 0.9832 & 0.6645 & 0.1998 & 0.6645 & 0.9832 & 0.9999 & 1.0000 & 1.0000 & 1.0000 \\
\hline 112. & 1.0000 & 1.0000 & 1.0000 & 1.0000 & 0.9931 & 0.7230 & 0.1998 & 0.7230 & 0.9931 & 1.0000 & 1.0000 & 1.0000 & 1.0000 \\
\hline 120. & 1.0000 & 1.0000 & 1.0000 & 1.0000 & 0.9978 & 0.7839 & 0.1998 & 0.7839 & 0.9978 & 1.0000 & 1.0000 & 1.0000 & 1.0000 \\
\hline 128. & 1.0000 & 1.0000 & 1.0000 & 1.0000 & 0.9995 & 0.8454 & 0.1998 & 0.8454 & 0.9995 & 1.0000 & 1.0000 & 1.0000 & 1.0000 \\
\hline 136. & 1.0000 & 1.0000 & 1.0000 & 1.0000 & 0.9999 & 0.9037 & 0.1998 & 0.9037 & 0.9999 & 1.0000 & 1.0000 & 1.0000 & 1.0000 \\
\hline 144. & 1.0000 & 1.0000 & 1.0000 & 1.0000 & 1.0000 & 0.9530 & 0.1998 & 0.9530 & 1.0000 & 1.0000 & 1.0000 & 1.0000 & 1.0000 \\
\hline 152. & 1.0000 & 1.0000 & 1.0000 & 1.0000 & 1.0000 & 0.9858 & 0.1998 & 0.9858 & 1.0000 & 1.0000 & 1.0000 & 1.0000 & 1.0000 \\
\hline 160. & 1.0000 & 1.0000 & 1.0000 & 1.0000 & 1.0000 & 0.9987 & 0.1998 & 0.9987 & 1.0000 & 1.0000 & 1.0000 & 1.0000 & 1.0000 \\
\hline
\end{tabular}




\section{TAHE A.41 THEATER/AUDITORIUM}

THE YOHER OF THE TEST OF TIE HYYOUILSIS THAT MX $=$ MO WIIEN THE SIGNIFICANCE LEVEL IS 0.10

\begin{tabular}{|c|c|c|c|c|c|c|c|c|c|c|c|c|c|}
\hline $\mathbf{N}$ & -15.00 & -12.50 & -10.00 & -7.50 & -5.00 & -2.50 & 0.00 & 2.50 & 5.00 & 7.50 & 10.00 & 12.50 & 15.00 \\
\hline 8. & 0.5891 & 0.4660 & 0.3470 & 0.2437 & 0.1651 & 0.1164 & 0.1000 & 0.1164 & 0.1651 & 0.2437 & 0.3470 & 0.4660 & 0.5891 \\
\hline 16. & 0.8562 & 0.7298 & 0.5641 & 0.3870 & 0.2344 & 0.1343 & 0.1000 & 0.1343 & 0.2344 & 0.3870 & 0.5641 & 0.7298 & 0.8562 \\
\hline 24. & 0.9606 & 0.8830 & 0.7335 & 0.5228 & 0.3074 & 0.1540 & 0.1000 & 0.1540 & 0.3074 & 0.5228 & 0.7335 & 0.8830 & 0.9606 \\
\hline 32. & 0.9916 & 0.9571 & 0.8520 & 0.6454 & 0.3835 & 0.1756 & 0.1000 & 0.1756 & 0.3835 & 0.6454 & 0.8520 & 0.9571 & 0.9916 \\
\hline 40. & 0.9986 & 0.9869 & 0.9263 & 0.7502 & 0.4616 & 0.1995 & 0.1000 & 0.1995 & 0.4616 & 0.7502 & 0.9263 & 0.9869 & 0.9986 \\
\hline 48. & 0.9998 & 0.9967 & 0.9676 & 0,8349 & 0.5404 & 0.2259 & 0.1000 & 0.2259 & 0.5404 & 0.8349 & 0.9676 & 0.9967 & 0.9998 \\
\hline 56. & 1.0000 & 0.9994 & 0.9877 & 0.8988 & 0.6186 & 0.2553 & 0.1000 & 0.2553 & 0.6186 & 0.8988 & 0.9877 & 0.9994 & 1.0000 \\
\hline 64. & 1.0000 & 0.9999 & 0.9961 & 0.9434 & 0.6942 & 0.2880 & 0.1000 & 0.2880 & 0.6942 & 0.9434 & 0.9961 & 0.9999 & 1.0000 \\
\hline 72. & 1.0000 & 1.0000 & 0.9990 & 0.9717 & 0.7651 & 0.3247 & 0.1000 & 0.3247 & 0.7651 & 0.9717 & 0.9990 & 1.0000 & 1.0000 \\
\hline 80 . & 1.0000 & 1.0000 & 0.9998 & 0.9877 & 0.8291 & 0.3659 & 0.1000 & 0.3659 & 0.8291 & 0.9877 & 0.9998 & 1.0000 & 1.0000 \\
\hline 88. & 1.0000 & 1.0000 & 1.0000 & 0.9956 & 0.8841 & 0.4122 & 0.1000 & 0.4122 & 0.8841 & 0.9956 & 1.0000 & 1.0000 & 1.0000 \\
\hline 96. & 1.0000 & 1.0000 & 1.0000 & 0.9987 & 0.9283 & 0.4645 & 0.1000 & 0.4645 & 0.9283 & 0.9987 & 1.0000 & 1.0000 & 1.0000 \\
\hline 104. & 1.0000 & 1.0000 & 1.0000 & 0.9997 & 0.9609 & 0.5235 & 0.1000 & 0.5235 & 0.9609 & 0.9997 & 1.0000 & 1.0000 & 1.0000 \\
\hline 112. & 1.0000 & 1.0000 & 1.0000 & 1.0000 & 0.9820 & 0.5897 & 0.1000 & 0.5897 & 0.9820 & 1.0000 & 1.0000 & 1.0000 & 1.0000 \\
\hline 120. & 1.0000 & 1.0000 & 1.0000 & 1.0000 & 0.9936 & 0.6633 & 0.1000 & 0.6633 & 0.9936 & 1.0000 & 1.0000 & 1.0000 & 1.0000 \\
\hline 128. & 1.0000 & 1.0000 & 1.0000 & 1.0000 & 0.9984 & 0.7433 & 0.1000 & 0.7433 & 0.9984 & 1.0000 & 1.0000 & 1.0000 & 1.0000 \\
\hline 136. & 1.0000 & 1.0000 & 1.0000 & 1.0000 & 0.9998 & 0.8264 & 0.1000 & 0.8264 & 0.9998 & 1.0000 & 1.0000 & 1.0000 & 1.0000 \\
\hline 144. & 1.0000 & 1.0000 & 1.0000 & 1.0000 & 1.0000 & 0.9051 & 0.1000 & 0.9051 & 1.0000 & 1.0000 & 1.0000 & 1.0000 & 1.0000 \\
\hline 152. & 1.0000 & 1.0000 & 1.0000 & 1.0000 & 1.0000 & 0.9662 & 0.1000 & 0.9662 & 1.0000 & 1.0000 & 1.0000 & 1.0000 & 1.0000 \\
\hline 160. & 1.0000 & 1.0000 & 1.0000 & 1.0000 & 1.0000 & 0.9958 & 0.1000 & 0.9958 & 1.0000 & 1.0000 & 1.0000 & 1.0000 & 1.0000 \\
\hline
\end{tabular}


TARE A.42 TIEATER/AUDI TORI UM

IHE POWER OF THE TEST OF THE HYYOIHESIS THAT MX $=$ MO WHEN THE SIGNIFICANCE LEVIA, IS 0.05

\begin{tabular}{|c|c|c|c|c|c|c|c|c|c|c|c|c|c|}
\hline \multirow[b]{2}{*}{ N } & \multicolumn{13}{|c|}{$(M X-M 0)$} \\
\hline & -15.00 & -12.50 & -10.00 & -7.50 & -5.00 & -2.50 & 0.00 & 2.50 & 5.00 & 7.50 & 10.00 & 12.50 & 15.00 \\
\hline 8. & 0.4641 & 0.3441 & 0.2384 & 0.1545 & 0.0955 & 0.0612 & 0.0500 & 0.061 .2 & 0.0955 & 0.1545 & 0.2384 & 0.3441 & 0.4641 \\
\hline 16. & 0.7729 & 0.6168 & 0.4388 & 0.2728 & 0.1473 & 0.0736 & 0.0500 & 0.0736 & 0.1473 & 0.2728 & 0.4388 & 0.6168 & 0.7729 \\
\hline 24. & 0.9254 & 0.8093 & 0.6211 & 0.3980 & 0.2054 & 0.0876 & 0.0500 & 0.0876 & 0.2054 & 0.3980 & 0.6211 & 0.8093 & 0.9254 \\
\hline 32. & 0.9810 & 0.9196 & 0.7673 & 0.5230 & 0.2697 & 0.1032 & 0.0500 & 0.1032 & 0.2697 & 0.5230 & 0.7673 & 0.9196 & 0.9810 \\
\hline 40. & 0.9963 & 0.9717 & 0.8715 & 0.6406 & 0.3399 & 0.1208 & 0.0500 & 0.1208 & 0.3399 & 0.6406 & 0.8715 & 0.971 .7 & 0.9963 \\
\hline 48. & 0.9995 & 0.9919 & 0.9372 & 0.7449 & 0.4153 & 0.1408 & 0.0500 & 0.1408 & 0.4153 & 0.7449 & 0.9372 & 0.9919 & 0.9995 \\
\hline 56. & 0.9999 & 0.9982 & 0.9734 & 0.8314 & 0.4946 & 0.1635 & 0.0500 & 0.1635 & 0.4946 & 0.8314 & 0.9734 & 0.9982 & 0.9999 \\
\hline 64. & 1.0000 & 0.9997 & 0.9905 & 0.8978 & 0.5764 & 0.1896 & 0.0500 & 0.1896 & 0.5764 & 0.8978 & 0.9905 & 0.9997 & 1.0000 \\
\hline 72 . & 1.0000 & 1.0000 & 0.9972 & 0.9443 & 0.6583 & 0.2197 & 0.0500 & 0.2197 & 0.6583 & 0.9443 & 0.9972 & 1.0000 & 1.0000 \\
\hline 80. & 1.0000 & 1.0000 & 0.9994 & 0.9734 & 0.7375 & 0.2545 & 0.0500 & 0.2545 & 0.7375 & 0.9734 & 0.9994 & 1.0000 & 1.0000 \\
\hline 88. & 1.0000 & 1.0000 & 0.9999 & 0.9893 & 0.8108 & 0.2951 & 0.0500 & 0.2951 & 0.8108 & 0.9893 & 0.9999 & 1.0000 & 1.0000 \\
\hline 96 . & 1.0000 & 1.0000 & 1.0000 & 0.9966 & 0.8746 & 0.3427 & 0.0500 & 0.3427 & 0.8746 & 0.9966 & 1.0000 & 1.0000 & 1.0000 \\
\hline 104. & 1.0000 & 1.0000 & 1.0000 & 0.9992 & 0.9259 & 0.3987 & 0.0500 & 0.3987 & 0.9259 & 0.9992 & 1.0000 & 1.0000 & 1.0000 \\
\hline 112. & 1.0000 & 1.0000 & 1.0000 & 0.9999 & 0.9627 & 0.4647 & 0.0500 & 0.4647 & 0.9627 & 0.9999 & 1.0000 & 1.0000 & 1.0000 \\
\hline 120. & 1.0000 & 1.0000 & 1.0000 & 1.0000 & 0.9851 & 0.5423 & 0.0500 & 0.5423 & 0.9851 & 1.0000 & 1.0000 & 1.0000 & 1.0000 \\
\hline 128. & 1.0000 & 1.0000 & 1.0000 & 1.0000 & 0.9958 & 0.6324 & 0.0500 & 0.6324 & 0.9958 & 1.0000 & 1.0000 & 1.0000 & 1.0000 \\
\hline 136. & 1.0000 & 1.0000 & 1.0000 & 1.0000 & 0.9993 & 0.7340 & 0.0500 & 0.7340 & 0.9993 & 1.0000 & 1.0000 & 1.0000 & 1.0000 \\
\hline 144. & 1.0000 & 1.0000 & 1.0000 & 1.0000 & 1.0000 & 0.8404 & 0.0500 & 0.8404 & 1.0000 & 1.0000 & 1.0000 & 1.0000 & 1.0000 \\
\hline 152. & 1.0000 & 1.0000 & 1.0000 & 1.0000 & 1.0000 & 0.9349 & 0.0500 & 0.9349 & 1.0000 & 1.0000 & 1.0000 & 1.0000 & 1.0000 \\
\hline 160. & 1.0000 & 1.0000 & 1.0000 & 1.0000 & 1.0000 & 0.9899 & 0.0500 & 0.9899 & 1.0000 & 1.0000 & 1.0000 & 1.0000 & 1.0000 \\
\hline
\end{tabular}


TABLE A.43 COMMUNITY CENTER

THE MOWER OF TIE TEST OF THE IYY POWLESIS THAT MX $=$ MO WILN THE SIGNIFICANCE LEVEL IS 0.20

\begin{tabular}{|c|c|c|c|c|c|c|c|c|c|c|c|c|c|}
\hline \multirow[b]{2}{*}{$\mathbf{N}$} & \multicolumn{13}{|c|}{$(M X-M O)$} \\
\hline & -15.00 & -12.50 & -10.00 & -7.50 & -5.00 & -2.50 & 0.00 & 2.50 & 5.00 & 7.50 & 10.00 & 12.50 & 15.00 \\
\hline 11. & 0.8472 & 0.7394 & 0.6031 & 0.4561 & 0.3240 & 0.2325 & 0.1998 & 0.2325 & 0.3240 & 0.4561 & 0.6031 & 0.7394 & 0.8472 \\
\hline 22 . & 0.9806 & 0.9343 & 0.8293 & 0.6542 & 0.4424 & 0.2674 & 0.1998 & 0.2674 & 0.4424 & 0.6542 & 0.8293 & 0.9343 & 0.9806 \\
\hline 33 . & 0.9983 & 0.9872 & 0.9370 & 0.7963 & 0.5530 & 0.3049 & 0.1998 & 0.3049 & 0.5530 & 0.7963 & 0.9370 & 0.9872 & 0.9983 \\
\hline 44. & 0.9999 & 0.9981 & 0.9805 & 0.8902 & 0.6536 & 0.3450 & 0.1998 & 0.3450 & 0.6536 & 0.8902 & 0.9805 & 0.9981 & 0.9999 \\
\hline 55. & 1.0000 & 0.9998 & 0.9950 & 0.9467 & 0.7423 & 0.3880 & 0.1998 & 0.3880 & 0.7423 & 0.9467 & 0.9950 & 0.9998 & 1.0000 \\
\hline 66. & 1.0000 & 1.0000 & 0.9990 & 0.9772 & 0.8177 & 0.4341 & 0.1998 & 0.4341 & 0.8177 & 0.9772 & 0.9990 & 1.0000 & 1.0000 \\
\hline 77. & 1.0000 & 1.0000 & 0.9999 & 0.9917 & 0.8787 & 0.4834 & 0.1998 & 0.4834 & 0.8787 & 0.9917 & 0.9999 & 1.0000 & 1.0000 \\
\hline 88. & 1.0000 & 1.0000 & 1.0000 & 0.9975 & 0.9254 & 0.5360 & 0.1998 & 0.5360 & 0.9254 & 0.9975 & 1.0000 & 1.0000 & 1.0000 \\
\hline 99. & 1.0000 & 1.0000 & 1.0000 & 0.9994 & 0.9584 & 0.5918 & 0.1998 & 0.5918 & 0.9584 & 0.9994 & 1.0000 & 1.0000 & 1.0000 \\
\hline 110. & 1.0000 & 1.0000 & 1.0000 & 0.9999 & 0.9797 & 0.6505 & 0.1998 & 0.6505 & 0.9797 & 0.9999 & 1.0000 & 1.0000 & 1.0000 \\
\hline 121. & 1.0000 & 1.0000 & 1.0000 & 1.0000 & 0.9916 & 0.7116 & 0.1998 & 0.7116 & 0.9916 & 1.0000 & 1.0000 & 1.0000 & 1.0000 \\
\hline 132. & 1.0000 & 1.0000 & 1.0000 & 1.0000 & 0.9973 & 0.7738 & 0.1998 & 0.7738 & 0.9973 & 1.0000 & 1.0000 & 1.0000 & 1.0000 \\
\hline 143. & 1.0000 & 1.0000 & 1.0000 & 1.0000 & 0.9994 & 0.8350 & 0.1998 & 0.8350 & 0.9994 & 1.0000 & 1.0000 & 1.0000 & 1.0000 \\
\hline 154. & 1.0000 & 1.0000 & 1.0000 & 1.0000 & 0.9999 & 0.8923 & 0.1998 & 0.8923 & 0.9999 & 1.0000 & 1.0000 & 1.0000 & 1.0000 \\
\hline 165. & 1.0000 & 1.0000 & 1.0000 & 1.0000 & 1.0000 & 0.9410 & 0.1998 & 0.9410 & 1.0000 & 1.0000 & 1.0000 & 1.0000 & 1.0000 \\
\hline 176. & 1.0000 & 1.0000 & 1.0000 & 1.0000 & 1.0000 & 0.9763 & 0.1998 & 0.9763 & 1.0000 & 1.0000 & 1.0000 & 1.0000 & 1.0000 \\
\hline 187. & 1.0000 & 1.0000 & 1.0000 & 1.0000 & 1.0000 & 0.9948 & 0.1998 & 0.9948 & 1.0000 & 1.0000 & 1.0000 & 1.0000 & 1.0000 \\
\hline 198. & 1.0000 & 1.0000 & 1.0000 & 1.0000 & 1.0000 & 0.9997 & 0.1998 & 0.9997 & 1.0000 & 1.0000 & 1.0000 & 1.0000 & 1.0000 \\
\hline 209. & 1.0000 & 1.0000 & 1.0000 & 1.0000 & 1.0000 & 1.0000 & 0.1998 & 1.0000 & 1.0000 & 1.0000 & 1.0000 & 1.0000 & 1.0000 \\
\hline 220 & 1.0000 & 1.0000 & 1.0000 & 1.0000 & 1.0000 & 1.0000 & 0.1998 & 1.0000 & 1.0000 & 1.0000 & 1.0000 & 1.0000 & 1.0000 \\
\hline
\end{tabular}


TAN.E A.44 COMMUNITY CENTER

THE POWER OI THE TEST OF THE IYIOTHESIS MIAT MX $=$ MO WHEN THE SIGNIFICANCE LEVG. IS 0.10

\begin{tabular}{|c|c|c|c|c|c|c|c|c|c|c|c|c|c|}
\hline \multirow[b]{2}{*}{$N$} & \multicolumn{13}{|c|}{$(M X-M O)$} \\
\hline & -15.00 & -12.50 & -10.00 & -7.50 & -5.00 & -2.50 & 0.00 & 2.50 & 5.00 & 7.50 & 10.00 & 12.50 & 15.00 \\
\hline 11. & 0.7456 & 0.6090 & 0.4578 & 0.3139 & 0.1983 & 0.1249 & 0.1000 & 0.1249 & 0.1983 & 0.3139 & 0.4578 & 0.6090 & 0.7456 \\
\hline 22. & 0.9558 & 0.8740 & 0.7216 & 0.5122 & 0.3014 & 0.1523 & 0.1000 & 0.1523 & 0.3014 & 0.5122 & 0.7216 & 0.8740 & 0.9558 \\
\hline 33. & 0.9950 & 0.9693 & 0.8784 & 0.6788 & 0.4067 & 0.1825 & 0.1000 & 0.1825 & 0.4067 & 0.6788 & 0.8784 & 0.9693 & 0.9950 \\
\hline 44. & 0.9996 & 0.9944 & 0.9555 & 0.8063 & 0.5115 & 0.2159 & 0.1000 & 0.2159 & 0.5115 & 0.8063 & 0.9555 & 0.9944 & 0.9996 \\
\hline 55. & 1.0000 & 0.9993 & 0.9867 & 0.8945 & 0.6125 & 0.2529 & 0.1000 & 0.2529 & 0.6125 & 0.8945 & 0.9867 & 0.9993 & 1.0000 \\
\hline 66. & 1.0000 & 0.9999 & 0.9968 & 0.9491 & 0.7064 & 0.2938 & 0.1000 & 0.2938 & 0.7064 & 0.9491 & 0.9968 & 0.9999 & 1.0000 \\
\hline 77. & 1.0000 & 1.0000 & 0.9994 & 0.9788 & 0.7897 & 0.3393 & 0.1000 & 0.3393 & 0.7897 & 0.9788 & 0.9994 & 1.0000 & 1.0000 \\
\hline 88. & 1.0000 & 1.0000 & 0.9999 & 0.9927 & 0.8597 & 0.3899 & 0.1000 & 0.3899 & 0.8597 & 0.9927 & 0.9999 & 1.0000 & 1.0000 \\
\hline 99. & 1.0000 & 1.0000 & 1.0000 & 0.9980 & 0.9146 & 0.4461 & 0.1000 & 0.4461 & 0.9146 & 0.9980 & 1.0000 & 1.0000 & 1.0000 \\
\hline 10. & 1.0000 & 1.0000 & 1.0000 & 0.9996 & 0.9539 & 0.5082 & 0.1000 & 0.5082 & 0.9539 & 0.9996 & 1.0000 & 1.0000 & 1.0000 \\
\hline 1. & 1.0000 & 1.0000 & 1.0000 & 0.9999 & 0.9788 & 0.5765 & 0.1000 & 0.5765 & 0.9788 & 0.9999 & 1.0000 & 1.0000 & 1.0000 \\
\hline 2. & 1.0000 & 1.0000 & 1.0000 & 1.0000 & 0.9922 & 0.6507 & 0.1000 & 0.6507 & 0.9922 & 1.0000 & 1.0000 & 1.0000 & 1.0000 \\
\hline 143. & 1.0000 & 1.0000 & 1.0000 & 1.0000 & 0.9979 & 0.7293 & 0.1000 & 0.7293 & 0.9979 & 1.0000 & 1.0000 & 1.0000 & 1.0000 \\
\hline 154. & 1.0000 & 1.0000 & 1.0000 & 1.0000 & 0.9997 & 0.8093 & 0.1000 & 0.8093 & 0.9997 & 1.0000 & 1.0000 & 1.0000 & 1.0000 \\
\hline 65. & 1.0000 & 1.0000 & 1.0000 & 1.0000 & 1.0000 & 0.8850 & 0.1000 & 0.8850 & 1.0000 & 1.0000 & 1.0000 & 1.0000 & 1.0000 \\
\hline 6. & 1.0000 & 1.0000 & 1.0000 & 1.0000 & 1.0000 & 0.9473 & 0.1000 & 0.9473 & 1.0000 & 1.0000 & 1.0000 & 1.0000 & 1.0000 \\
\hline 187. & 1.0000 & 1.0000 & 1.0000 & 1.0000 & 1.0000 & 0.9861 & 0.1000 & 0.9861 & 1.0000 & 1.0000 & 1.0000 & 1.0000 & 1.0000 \\
\hline 198. & 1.0000 & 1.0000 & 1.0000 & 1.0000 & 1.0000 & 0.9990 & 0.1000 & 0.9990 & 1.0000 & 1.0000 & 1.0000 & 1.0000 & 1.0000 \\
\hline 9. & 1.0000 & 1.0000 & 1.0000 & 1.0000 & 1.0000 & 1.0000 & 0.1000 & 1.0000 & 1.0000 & 1.0000 & 1.0000 & 1.0000 & 1.0000 \\
\hline & 1.0000 & 1.0000 & 1.0000 & 1.0000 & 1.0000 & 1.0000 & 0.1000 & 1.0000 & 1.0000 & 1.0000 & 1.0000 & 1.0000 & 1.0000 \\
\hline
\end{tabular}


TAIULE A.45 COMHUNITY CENIER

ITIE POWER OF TIIE TEST OF TUI: HY IU'TIESIS TUAT MX = MO WILN TIE SIGNIFICANCE LEVEL IS 0.05

\begin{tabular}{|c|c|c|c|c|c|c|c|c|c|c|c|c|c|}
\hline \multirow[b]{2}{*}{$\mathbf{N}$} & \multicolumn{13}{|c|}{$(M X-M O)$} \\
\hline & -15.00 & -12.50 & -10.00 & -7.50 & -5.00 & -2.50 & 0.00 & 2.50 & 5.00 & 7.50 & 10.00 & 12.50 & 15.00 \\
\hline 11. & 0.6352 & 0.4846 & 0.3364 & 0.2107 & 0.1199 & 0.0671 & 0.0500 & 0.0671 & 0.1199 & 0.2107 & 0.3364 & 0.4846 & 0.6352 \\
\hline 22 . & 0.9175 & 0.7969 & 0.6074 & 0.3877 & 0.2004 & 0.0864 & 0.0500 & 0.0864 & 0.2004 & 0.3877 & 0.6074 & 0.7969 & 0.9175 \\
\hline 33. & 0.9880 & 0.9401 & 0.8030 & 0.5593 & 0.2902 & 0.1083 & 0.0500 & 0.1083 & 0.2902 & 0.5593 & 0.8030 & 0.9401 & 0.9880 \\
\hline 44. & 0.9989 & 0.9869 & 0.9171 & 0.7086 & 0.3871 & 0.1332 & 0.0500 & 0.1332 & 0.3871 & 0.7086 & 0.9171 & 0.9869 & 0.9989 \\
\hline 55. & 0.9999 & 0.9979 & 0.9713 & 0.8253 & 0.4883 & 0.1616 & 0.0500 & 0.1616 & 0.4883 & 0.8253 & 0.9713 & 0.9979 & 0.9999 \\
\hline 66. & 1.0000 & 0.9998 & 0.9921 & 0.9068 & 0.5900 & 0.1943 & 0.0500 & 0.1943 & 0.5900 & 0.9068 & 0.9921 & 0.9998 & 1.0000 \\
\hline 77. & 1.0000 & 1.0000 & 0.9984 & 0.9569 & 0.6880 & 0.2319 & 0.0500 & 0.2319 & 0.6880 & 0.9569 & 0.9984 & 1.0000 & 1.0000 \\
\hline 88. & 1.0000 & 1.0000 & 0.9998 & 0.9833 & 0.7776 & 0.2754 & 0.0500 & 0.2754 & 0.7776 & 0.9833 & 0.9998 & 1.0000 & 1.0000 \\
\hline 99. & 1.0000 & 1.0000 & 1.0000 & 0.9948 & 0.8542 & 0.3257 & 0.0500 & 0.3257 & 0.8542 & 0.9948 & 1.0000 & 1.0000 & 1.0000 \\
\hline 110. & 1.0000 & 1.0000 & 1.0000 & 0.9988 & 0.9145 & 0.3839 & 0.0500 & 0.3839 & 0.9145 & 0.9988 & 1.0000 & 1.0000 & 1.0000 \\
\hline 121. & 1.0000 & 1.0000 & 1.0000 & 0.9998 & 0.9568 & 0.4513 & 0.0500 & 0.4513 & 0.9568 & 0.9998 & 1.0000 & 1.0000 & 1.0000 \\
\hline 132. & 1.0000 & 1.0000 & 1.0000 & 1.0000 & 0.9823 & 0.5286 & 0.0500 & 0.5286 & 0.9823 & 1.0000 & 1.0000 & 1.0000 & 1.0000 \\
\hline 143. & 1.0000 & 1.0000 & 1.0000 & 1.0000 & 0.9946 & 0.6162 & 0.0500 & 0.6162 & 0.9946 & 1.0000 & 1.0000 & 1.0000 & 1.0000 \\
\hline 154. & 1.0000 & 1.0000 & 1.0000 & 1.0000 & 0.9990 & 0.7124 & 0.0500 & 0.7124 & 0.9990 & 1.0000 & 1.0000 & 1.0000 & 1.0000 \\
\hline 165. & 1.0000 & 1.0000 & 1.0000 & 1.0000 & 0.9999 & 0.8120 & 0.0500 & 0.8120 & 0.9999 & 1.0000 & 1.0000 & 1.0000 & 1.0000 \\
\hline 176. & 1.0000 & 1.0000 & 1.0000 & 1.0000 & 1.0000 & 0.9040 & 0.0500 & 0.9040 & 1.0000 & 1.0000 & 1.0000 & 1.0000 & 1.0000 \\
\hline 187. & 1.0000 & 1.0000 & 1.0000 & 1.0000 & 1.0000 & 0.9703 & 0.0500 & 0.9703 & 1.0000 & 1.0000 & 1.0000 & 1.0000 & 1.0000 \\
\hline 198. & 1.0000 & 1.0000 & 1.0000 & 1.0000 & 1.0000 & 0.9973 & 0.0500 & 0.9973 & 1.0000 & 1.0000 & 1.0000 & 1.0000 & 1.0000 \\
\hline 209. & 1.0000 & 1.0000 & 1.0000 & 1.0000 & 1.0000 & 1.0000 & 0.0500 & 1.0000 & 1.0000 & 1.0000 & 1.0000 & 1.0000 & 1.0000 \\
\hline 220 . & 1.0000 & 1.0000 & 1.0000 & 1.0000 & 1.0000 & 1.0000 & 0.0500 & 1.0000 & 1.0000 & 1.0000 & 1.0000 & 1.0000 & 1.0000 \\
\hline
\end{tabular}


TAILE A.46 GYMNASIUM

IHE POWER OF THE TEST OF THE HY PUTHESIS THAT MX = MO WIEN THE SIGNIFICANCE LEVES, IS 0.20

\begin{tabular}{|c|c|c|c|c|c|c|c|c|c|c|c|c|c|}
\hline \multirow[b]{2}{*}{$\mathbf{N}$} & \multicolumn{13}{|c|}{$(M X-M O)$} \\
\hline & -15.00 & -12.50 & -10.00 & -7.50 & -5.00 & -2.50 & 0.00 & 2.50 & 5.00 & 7.50 & 10.00 & 12.50 & 15.00 \\
\hline 25. & 0.8134 & 0.7021 & 0.5690 & 0.4312 & 0.3108 & 0.2289 & 0.1998 & 0.2289 & 0.3108 & 0.4312 & 0.5690 & 0.7021 & 0.8134 \\
\hline 50. & 0.9686 & 0.9094 & 0.7923 & 0.6160 & 0.4173 & 0.2596 & 0.1998 & 0.2596 & 0.4173 & 0.6160 & 0.7923 & 0.9094 & 0.9686 \\
\hline 75. & 0.9961 & 0.9775 & 0.9111 & 0.7554 & 0.5177 & 0.2923 & 0.1998 & 0.2923 & 0.5177 & 0.7554 & 0.9111 & 0.9775 & 0.9961 \\
\hline 100. & 0.9996 & 0.9955 & 0.9665 & 0.8541 & 0.6107 & $0.32 \%$ & 0.1998 & 0.3270 & 0.6107 & 0.8541 & 0.9665 & 0.9955 & 0.9996 \\
\hline 125. & 1.0000 & 0.9993 & 0.9891 & 0.9193 & 0.6947 & 0.3638 & 0.1998 & 0.3638 & 0.6947 & 0.9193 & 0.9891 & 0.9993 & 1.0000 \\
\hline 150. & 1.0000 & 0.9999 & 0.9970 & 0.9592 & 0.7688 & 0.4029 & 0.1998 & 0.4029 & 0.7688 & 0.9592 & 0.9970 & 0.9999 & 1.0000 \\
\hline 175. & 1.0000 & 1.0000 & 0.9993 & 0.9814 & 0.8320 & 0.4444 & 0.1998 & 0.4444 & 0.8320 & 0.9814 & 0.9993 & 1.0000 & 1.0000 \\
\hline 200 & 1.0000 & 1.0000 & 0.9999 & 0.9925 & 0.8839 & 0.4884 & 0.1998 & 0.4884 & 0.8839 & 0.9925 & 0.9999 & 1.0000 & 1.0000 \\
\hline 225 . & 1.0000 & 1.0000 & 1.0000 & 0.9974 & 0.9245 & 0.5348 & 0.1998 & 0.5348 & 0.9245 & 0.9974 & 1.0000 & 1.0000 & 1.0000 \\
\hline 250 . & 1.0000 & 1.0000 & 1.0000 & 0.9993 & 0.9545 & 0.5837 & 0.1998 & 0.5837 & 0.9545 & 0.9993 & 1.0000 & 1.0000 & 1.0000 \\
\hline 275. & 1.0000 & 1.0000 & 1.0000 & 0.9998 & 0.9751 & 0.6348 & 0.1998 & 0.6348 & 0.9751 & 0.9998 & 1.0000 & 1.0000 & 1.0000 \\
\hline 300. & 1.0000 & 1.0000 & 1.0000 & 1.0000 & 0.9879 & 0.6877 & 0.1998 & 0.6877 & 0.9879 & 1.0000 & 1.0000 & 1.0000 & 1.0000 \\
\hline 325 . & 1.0000 & 1.0000 & 1.0000 & 1.0000 & 0.9950 & 0.7419 & 0.1998 & 0.7419 & 0.9950 & 1.0000 & 1.0000 & 1.0000 & 1.0000 \\
\hline 350. & 1.0000 & 1.0000 & 1.0000 & 1.0000 & 0.9983 & 0.7962 & 0.1998 & 0.7962 & 0.9983 & 1.0000 & 1.0000 & 1.0000 & 1.0000 \\
\hline 375. & 1.0000 & 1.0000 & 1.0000 & 1.0000 & 0.9996 & 0.8490 & 0.1998 & 0.8490 & 0.9996 & 1.0000 & 1.0000 & 1.0000 & 1.0000 \\
\hline 400 & 1.0000 & 1.0000 & 1.0000 & 1.0000 & 0.9999 & 0.8980 & 0.1998 & 0.8980 & 0.9999 & 1.0000 & 1.0000 & 1.0000 & 1.0000 \\
\hline 425. & 1.0000 & 1.0000 & 1.0000 & 1.0000 & 1.0000 & 0.9400 & 0.1998 & 0.9400 & 1.0000 & 1.0000 & 1.0000 & 1.0000 & 1.0000 \\
\hline 450 . & 1.0000 & 1.0000 & 1.0000 & 1.0000 & 1.0000 & 0.9719 & 0.1998 & 0.9719 & 1.0000 & 1.0000 & 1.0000 & 1.0000 & 1.0000 \\
\hline 475 . & 1.0000 & 1.0000 & 1.0000 & 1.0000 & 1.0000 & 0.9912 & 0.1998 & 0.9912 & 1.0000 & 1.0000 & 1.0000 & 1.0000 & 1.0000 \\
\hline 500 & 1.0000 & 1.0000 & 1.0000 & 1.0000 & 1.0000 & 0.9988 & 0.1998 & 0.9988 & 1.0000 & 1.0000 & 1.0000 & 1.0000 & 1.0000 \\
\hline
\end{tabular}


TAHLE A.47 GYMNASIUM

THE POWER OF THE TEST OF THL IIYIVTHESIS THAT MX = MO WHEN THE SIGNIFICANCE LUivid IS 0.10

\begin{tabular}{|c|c|c|c|c|c|c|c|c|c|c|c|c|c|}
\hline \multirow[b]{2}{*}{$\mathbf{N}$} & \multicolumn{13}{|c|}{$(M X-M O)$} \\
\hline & -15.00 & -12.50 & -10.00 & -7.50 & -5.00 & -2.50 & 0.00 & 2.50 & 5.00 & 7.50 & 10.00 & 12.50 & 15.00 \\
\hline 25 . & 0.7008 & 0.5656 & 0.4228 & 0.2912 & 0.1874 & 0.1221 & 0.1000 & 0.1221 & 0.1874 & 0.2912 & 0.4228 & 0.5656 & 0.7008 \\
\hline 50. & 0.9329 & 0.8350 & 0.6738 & 0.4713 & 0.2787 & 0.1462 & 0.1000 & 0.1462 & 0.2787 & 0.4713 & 0.6738 & 0.8350 & 0.9329 \\
\hline 75. & 0.9892 & 0.9496 & 0.8375 & 0.6283 & 0.3721 & 0.1723 & 0.1000 & 0.1723 & 0.3721 & 0.6283 & 0.8375 & 0.9496 & 0.9892 \\
\hline 100. & 0.9988 & 0.9877 & 0.9291 & 0.7552 & 0.4657 & 0.2008 & 0.1000 & 0.2008 & 0.4657 & 0.7552 & 0.9291 & 0.9877 & 0.9988 \\
\hline 125. & 0.9999 & 0.9976 & 0.9733 & 0.8502 & 0.5573 & 0.2319 & 0.1000 & 0.2319 & 0.5573 & 0.8502 & 0.9733 & 0.9976 & 0.9999 \\
\hline 150. & 1.0000 & 0.9997 & 0.9915 & 0.9159 & 0.6446 & 0.2660 & 0.1000 & 0.2660 & 0.6446 & 0.9159 & 0.9915 & 0.9997 & 1.0000 \\
\hline 175. & 1.0000 & 1.0000 & 0.9978 & 0.9573 & 0.7253 & 0.3032 & 0.1000 & 0.3032 & 0.7253 & 0.9573 & 0.9978 & 1.0000 & 1.0000 \\
\hline 200. & 1.0000 & 1.0000 & 0.9995 & 0.9807 & 0.7971 & 0.3440 & 0.1000 & 0.3440 & 0.7971 & 0.9807 & 0.9995 & 1.0000 & 1.0000 \\
\hline 225 . & 1.0000 & 1.0000 & 0.9999 & 0.9925 & 0.8584 & 0.3888 & 0.1000 & 0.3888 & 0.8584 & 0.9925 & 0.9999 & 1.0000 & 1.0000 \\
\hline 250. & 1.0000 & 1.0000 & 1.0000 & 0.9975 & 0.9078 & 0.4377 & 0.1000 & 0.4377 & 0.9078 & 0.9975 & 1.0000 & 1.0000 & 1.0000 \\
\hline 275 . & 1.0000 & 1.0000 & 1.0000 & 0.9994 & 0.9450 & 0.4912 & 0.1000 & 0.4912 & 0.9450 & 0.9994 & 1.0000 & 1.0000 & 1.0000 \\
\hline 300 . & 1.0000 & 1.0000 & 1.0000 & 0.9999 & 0.9707 & 0.5494 & 0.1000 & 0.5494 & 0.9707 & 0.9999 & 1.0000 & 1.0000 & 1.0000 \\
\hline 325. & 1.0000 & 1.0000 & 1.0000 & 1.0000 & 0.9866 & 0.6121 & 0.1000 & 0.6121 & 0.9866 & 1.0000 & 1.0000 & 1.0000 & 1.0000 \\
\hline 350. & 1.0000 & 1.0000 & 1.0000 & 1.0000 & 0.9950 & 0.6788 & 0.1000 & 0.6788 & 0.9950 & 1.0000 & 1.0000 & 1.0000 & 1.0000 \\
\hline 375. & 1.0000 & 1.0000 & 1.0000 & 1.0000 & 0.9986 & 0.7481 & 0.1000 & 0.7481 & 0.9986 & 1.0000 & 1.0000 & 1.0000 & 1.0000 \\
\hline 400 . & 1.0000 & 1.0000 & 1.0000 & 1.0000 & 0.9997 & 0.8177 & 0.1000 & 0.8177 & 0.9997 & 1.0000 & 1.0000 & 1.0000 & 1.0000 \\
\hline 425. & 1.0000 & 1.0000 & 1.0000 & 1.0000 & 1.0000 & 0.8834 & 0.1000 & 0.8834 & 1.0000 & 1.0000 & 1.0000 & 1.0000 & 1.0000 \\
\hline 450 . & 1.0000 & 1.0000 & 1.0000 & 1.0000 & 1.0000 & 0.9390 & 0.1000 & 0.9390 & 1.0000 & 1.0000 & 1.0000 & 1.0000 & 1.0000 \\
\hline 475 . & 1.0000 & 1.0000 & 1.0000 & 1.0000 & 1.0000 & 0.9778 & 0.1000 & 0.9778 & 1.0000 & 1.0000 & 1.0000 & 1.0000 & 1.0000 \\
\hline 500 & 1.0000 & 1.0000 & 1.0000 & 1.0000 & 1.0000 & 0.9961 & 0.1000 & 0.9961 & 1.0000 & 1.0000 & 1.0000 & 1.0000 & 1.0000 \\
\hline
\end{tabular}


TABLE A.48 GYMNASIUM

7IE POHER OF THE TEST OF THE HXYOTHESIS THAT MX $=$ MO WHEN THE SIGNIFICANCE LEVEL IS 0.05

\begin{tabular}{|c|c|c|c|c|c|c|c|c|c|c|c|c|c|}
\hline \multirow[b]{2}{*}{$\mathbf{N}$} & \multicolumn{13}{|c|}{$(M X-M O)$} \\
\hline & -15.00 & -12.50 & -10.00 & -7.50 & -5.00 & -2.50 & 0.00 & 2.50 & 5.00 & 7.50 & 10.00 & 12.50 & 15.00 \\
\hline 25 . & 0.5838 & 0.4403 & 0.3045 & 0.1922 & 0.1118 & 0.0651 & 0.0500 & 0.0651 & 0.1118 & 0.1922 & 0.3045 & 0.4403 & 0.5838 \\
\hline 50 . & 0.8816 & 0.7451 & 0.5538 & 0.3490 & 0.1821 & 0.0820 & 0.0500 & 0.0820 & 0.1821 & 0.3490 & 0.5538 & 0.7451 & 0.8816 \\
\hline 75. & 0.9764 & 0.9076 & 0.7483 & 0.5048 & 0.2599 & 0.1008 & 0.0500 & 0.1008 & 0.2599 & 0.5048 & 0.7483 & 0.9076 & 0.9764 \\
\hline 100 & 0.9966 & 0.9734 & 0.8758 & 0.6464 & 0.3437 & 0.1218 & 0.0500 & 0.1218 & 0.3437 & 0.6464 & 0.8758 & 0.9734 & 0.9966 \\
\hline 125. & 0.9997 & 0.9940 & 0.9470 & 0.7650 & 0.4319 & 0.1454 & 0.0500 & 0.1454 & 0.4319 & 0.7650 & 0.9470 & 0.9940 & 0.9997 \\
\hline 150. & 1.0000 & 0.9990 & 0.9808 & 0.8561 & 0.5221 & 0.1720 & 0.0500 & 0.1720 & 0.5221 & 0.8561 & 0.9808 & 0.9990 & 1.0000 \\
\hline 175. & 1.0000 & 0.9999 & 0.9942 & 0.9200 & 0.6116 & 0.2020 & 0.0500 & 0.2020 & 0.6116 & 0.9200 & 0.9942 & 0.9999 & 1.0000 \\
\hline 200 . & 1.0000 & 1.0000 & 0.9986 & 0.9603 & 0.6972 & 0.2359 & 0.0500 & 0.2359 & 0.6972 & 0.9603 & 0.9986 & 1.0000 & 1.0000 \\
\hline 225 . & 1.0000 & 1.0000 & 0.9997 & 0.9829 & 0.7758 & 0.2744 & 0.0500 & 0.2744 & 0.7758 & 0.9829 & 0.9997 & 1.0000 & 1.0000 \\
\hline 250 . & 1.0000 & 1.0000 & 1.0000 & 0.9938 & 0.8443 & 0.3181 & 0.0500 & 0.3181 & 0.8443 & 0.9938 & 1.0000 & 1.0000 & 1.0000 \\
\hline 275 . & 1.0000 & 1.0000 & 1.0000 & 0.9982 & 0.9003 & 0.3677 & 0.0500 & 0.3677 & 0.9003 & 0.9982 & 1.0000 & 1.0000 & 1.0000 \\
\hline 300. & 1.0000 & 1.0000 & 1.0000 & 0.9996 & 0.9425 & 0.4241 & 0.0500 & 0.4241 & 0.9425 & 0.9996 & 1.0000 & 1.0000 & 1.0000 \\
\hline 325. & 1.0000 & 1.0000 & 1.0000 & 0.9999 & 0.9712 & 0.4878 & 0.0500 & 0.4878 & 0.9712 & 0.9999 & 1.0000 & 1.0000 & 1.0000 \\
\hline 350. & 1.0000 & 1.0000 & 1.0000 & 1.0000 & 0.9880 & 0.5592 & 0.0500 & 0.5592 & 0.9880 & 1.0000 & 1.0000 & 1.0000 & 1.0000 \\
\hline 375. & 1.0000 & 1.0000 & 1.0000 & 1.0000 & 0.9962 & 0.6382 & 0.0500 & 0.6382 & 0.9962 & 1.0000 & 1.0000 & 1.0000 & 1.0000 \\
\hline 400 & 1.0000 & 1.0000 & 1.0000 & 1.0000 & 0.9992 & 0.7230 & 0.0500 & 0.7230 & 0.9992 & 1.0000 & 1.0000 & 1.0000 & 1.0000 \\
\hline 425. & 1.0000 & 1.0000 & 1.0000 & 1.0000 & 0.9999 & 0.8097 & 0.0500 & 0.8097 & 0.9999 & 1.0000 & 1.0000 & 1.0000 & 1.0000 \\
\hline 450 & 1.0000 & 1.0000 & 1.0000 & 1.0000 & 1.0000 & 0.8909 & 0.0500 & 0.8909 & 1.0000 & 1.0000 & 1.0000 & 1.0000 & 1.0000 \\
\hline 475 . & 1.0000 & 1.0000 & 1.0000 & 1.0000 & 1.0000 & 0.9550 & 0.0500 & 0.9550 & 1.0000 & 1.0000 & 1.0000 & 1.0000 & 1.0000 \\
\hline 500 & 1.0000 & 1.0000 & 1.0000 & 1.0000 & 1.0000 & 0.9906 & 0.0500 & 0.9906 & 1.0000 & 1.0000 & 1.0000 & 1.0000 & 1.0000 \\
\hline
\end{tabular}




\section{APPENDIX B}

\section{NUMBER OF BUILDINGS IN EACH STRATUM}

This Appendix gives a contingency table for each building type showing the number of buildings listed on the $1980 \mathrm{~F} . W$. Dodge Tapes that are in each of the 38 strata. The row totals show the number of buildings in each Census Division. The column totals show the number of buildings in each climate zone. Non-existent strata are identified by the symbol -, while a 0 indicates that the strata exists, but there were no construction starts or plans for 1980 . 
. 
Large Office

Census Division

Climate Zones

$\underline{\text { Totals }}$

\begin{tabular}{llllll}
1 & 2 & 3 & 4 & 6 & 7 \\
\hline
\end{tabular}

New Engl and (NE)

$142 \quad-\quad-\quad-\quad-$

43

Mid-Atlantic (NE)

$\begin{array}{llllll}0 & 53 & 54 & - & - & -\end{array}$

107

So. Atlantic (S)

$\begin{array}{llllll}0 & 0 & 69 & 49 & 47 & 10\end{array}$

175

E. So. Central ( S)

$\begin{array}{lllll}- & -10 & 14 & 2 & 1\end{array}$

27

W. So. Central ( S)

$\begin{array}{llllll}- & - & 0 & 32 & 105 & 65\end{array}$

202

E. No. Central (NC)

$\begin{array}{llllll}16 & 98 & 14 & - & - & -\end{array}$

128

W. No. Central (NC)

$\begin{array}{lll}18 & 10 & 31\end{array}$

$0 \quad-$

59

Mounta in

(W)

5

40

21

83

Pacific

(W)

3124

$24 \quad 177$

46

215

Totals

$\begin{array}{llllll}43 & 250 & 206 & 272 & 179 & 89\end{array}$

1039 
Sma11 Office

Census Division

Climate Zones

New Engl and (NE)

$43 \quad 182 \quad-\quad-\quad-\quad-$

225

Mid-Atrantic (NE)

$\begin{array}{lllll}10 & 230 & 237 & - & -\end{array}$

477

So. Atrantic (S)

$\begin{array}{llllll}9 & 5 & 345 & 576 & 969 & 91\end{array}$

1995

E. So. Central ( S)

$\begin{array}{llll}-\quad & - & 118 \quad 198 \quad 58 \quad 49\end{array}$

423

W. So. Central ( $S$ )

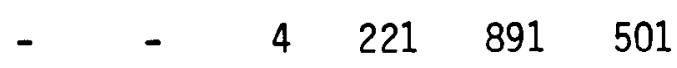

1617

E. No. Central (NC)

$203 \quad 1072 \quad 194$

1469

W. No. Central (NC)

$185 \quad 156 \quad 189$

530

Mounta in

(w)

$97 \quad 293 \quad 62$

799

Pacific

(w)

$\begin{array}{llll}72 & 41 & 294 & 1299\end{array}$

$32 \quad 127$

1865

Totals

$\begin{array}{llllll}619 & 1979 & 1443 & 2335 & 2198 & 826\end{array}$

9400 
High-Rise Apartment

Census Division

New Engl and

Mid-Atlantic

(NE)

So. Atlantic (S)

E. So. Central ( S)

W. So. Central ( S)

E. No. Central (NC)

W. No. Central (NC)

Mountain

Pacific

(w)

(w)

$15 \quad 21$

$3 \quad 0 \quad 34$

84
Totals

\begin{tabular}{llllll}
1 & 2 & 3 & 4 & 6 & 7 \\
\hline
\end{tabular}

75

125

493

30

36

120

51

42

143

Totals

$\begin{array}{llllll}67 & 253 & 194 & 124 & 454 & 23\end{array}$

1115 
Low-Rise Apartment

Census Division

Climate Zones

Totals

\begin{tabular}{lllllll}
1 & 2 & 3 & 4 & 6 & 7 \\
\hline
\end{tabular}

New Engl and

(NE) $\quad 111 \quad 229$

340

Mid-Atlantic (NE) $\quad 27 \quad 241 \quad 244 \quad-\quad$ -

512

So. Atlantic

7

$\begin{array}{lllll}8 & 354 & 682 & 2218 & 155\end{array}$

3424

E. So. Central ( $S$

- $\quad-201$

$215 \quad 68 \quad 86$

570

W. So. Central ( S

$-\quad-$

$\begin{array}{llll}3 & 190 & 581 & 304\end{array}$

1078

E. No. Central (NC) $4091261285 \quad-\quad-$

1955

W. No. Central (NC) $\begin{array}{lllllll}438 & 228 & 308 & 2 & -\end{array}$

976

Mountain

(w) $\quad 161 \quad 340$

69

$\begin{array}{lll}40 & 508 & 74\end{array}$

1192

Pacific

(w) 102

$43 \quad 461 \quad 2354$

$32 \quad 214$

3206

Totals 


\section{Elementary School}

Census Division

\begin{tabular}{llllll}
\multicolumn{3}{l}{ Cl imate Zones } \\
\cline { 2 - 4 }
\end{tabular}

Totals

New England (NE)

$81-\quad-\quad-$

Mid-Atrantic (NE)

0

So. Atlantic (S)

$\begin{array}{llllll}0 & 0 & 18 & 24 & 23 & 3\end{array}$

68

E. So. Central ( S)

$\begin{array}{llllll}- & - & 5 & 11 & 3 & 1\end{array}$

20

W. So. Central (S)

$\begin{array}{llll}- & 1 & 18 & 21\end{array}$

70

E. No. Central (NC)

$\begin{array}{llllll}5 & 24 & 6 & - & -\end{array}$

35

W. No. Central (NC)

$\begin{array}{llllll}12 & 9 & 15 & 0 & -\end{array}$

Mountain

( w)

$16 \quad 20$

Pacific

(w)

14

20

22

93

52

68

Totals

$\begin{array}{llllll}55 & 71 & 72 & 73 & 59 & 41\end{array}$

371 
Secondary School

Census Division

\begin{tabular}{llllll}
\multicolumn{7}{l}{ Climate Zones } \\
\cline { 2 - 4 }
\end{tabular}

Totals

New Engl and

(NE)

$74 \quad-$

11

Mid-Atlantic (NE)

$111 \quad 16$

28

So. Atlantic (S)

$\begin{array}{llllll}0 & 0 & 19 & 29 & 32 & 11\end{array}$

91

E. So. Central ( S )

$\begin{array}{lllll}- & - & 9 & 22 & 7\end{array}$

48

W. So. Central ( S

$\begin{array}{lllll}- & - & 3 & 34 & 48\end{array}$

167

E. No. Central (NC)

$12 \quad 46 \quad 14$

72

W. No. Central (NC)

$\begin{array}{lllll}10 & 12 & 22 & 0 & -\end{array}$

44

Mounta in

(w)

$19 \quad 23$

$\begin{array}{lll}3 & 7 & 12\end{array}$

1

65

Pacific

(w)

$18 \quad 10$

$21 \quad 16$

5

0

70

Totals 


\section{Clinic}

Census Division

Climate Zones

Totals

\begin{tabular}{llllll}
1 & 2 & 3 & 4 & 6 & 7 \\
\hline
\end{tabular}

New Engl and (NE) $\quad \begin{array}{llllll} & & 39 & - & - & -\end{array}$

Mid-Atlantic (NE) $\quad 8 \quad 64 \quad 58 \quad-\quad$ -

130

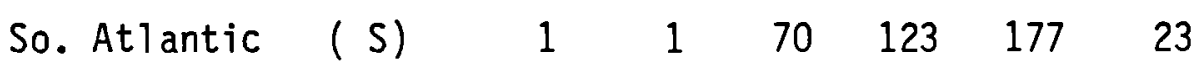

395

E. So. Central ( S) - $\quad$ - $\quad \begin{array}{lllll}42 & 72 & 15 & 20\end{array}$

149

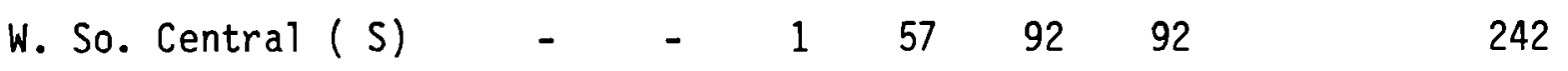

E No. Central (NC) $\quad \begin{array}{lllllll}56 & 227 & 50 & - & - & - & 333\end{array}$

$\begin{array}{llllllll}\text { W. No. Central (NC) } & 46 & 26 & 76 & 0 & - & - & 148\end{array}$

$\begin{array}{lllllllll}\text { Mountain } & \text { ( W }) & 13 & 16 & 10 & 7 & 24 & 1 & 71\end{array}$

$\begin{array}{lllllllll}\text { Pacific } & \text { ( W }) & 15 & 2 & 42 & 109 & 0 & 13 & 181\end{array}$

Totals

$\begin{array}{llllll}153 & 375 & 349 & 368 & 308 & 149\end{array}$

1702 
Hospital

Census Division

Climate Zones

Totals

\begin{tabular}{llllll}
1 & 2 & 3 & 4 & 6 & 7 \\
\hline
\end{tabular}

New Engl and

(NE)

1

Mid-Atlantic

0

So. Atlantic

( S)

0

0

$\begin{array}{lll}5 & 14 & 10\end{array}$

2

E. So. Central (S) - $\quad$ - $\quad \begin{array}{lllll}4 & 2 & 1 & 2\end{array}$

W. So. Central (S) - $\quad-\quad \begin{array}{lllll}6 & 0 & 6 & 9 & 3\end{array}$

18

E. No. Central (NC) $\quad \begin{array}{llllll}1 & 21 & 5 & - & - & -\end{array}$

W. No. Central (NC) $\quad \begin{array}{lllllll}4 & 4 & 2 & 0 & -\end{array}$

10

Mountain

(w) $\quad \begin{array}{llllll}6 & 7 & 0 & 1 & 1 & 1\end{array}$

16

Pacific

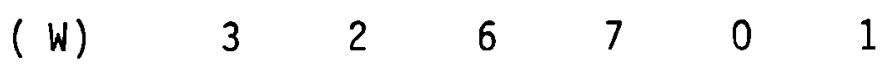

19

Totals

$\begin{array}{llllll}15 & 43 & 26 & 30 & 21 & 9\end{array}$ 
Nursing Home*

Census Division

$1 \quad 2$\begin{tabular}{llll}
$\frac{\text { Climate Zones }}{3}$ & & \\
\hline
\end{tabular}

$\underline{\text { Totals }}$

New England (NE) $\quad 0 \quad 0 \quad-\quad-\quad-\quad-$

Mid-Atlantic (NE) $\quad 0 \quad 0 \quad 0 \quad-\quad-$

So. Atlantic $(S) \quad 0 \quad 00 \begin{array}{lllll} & (S) & 0 & 0 & 0\end{array}$

E. So. Central (S) - $\quad-\quad \begin{array}{llllll} & 0 & 0 & 0 & 0 & 0\end{array}$

W. So. Central (S) - $\quad-\quad \begin{array}{llllll} & 0 & 0 & 0 & 0 & 0\end{array}$

E No. Central (NC) $0 \quad 0 \quad 0 \quad-\quad-\quad-\quad 0$

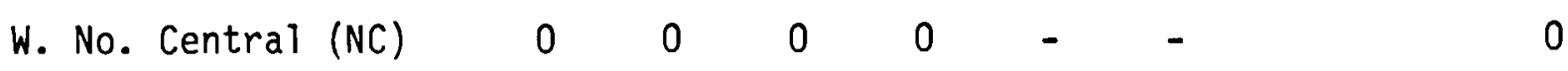

$\begin{array}{lllllllll}\text { Mountain } & (w) & 0 & 0 & 0 & 0 & 0 & 0 & 0\end{array}$

$\begin{array}{lllllllll}\text { Pacific } & (\mathrm{W}) & 1 & 4 & 6 & 7 & 1 & 0 & 19\end{array}$

$\begin{array}{llllllll}\text { Totals } & 1 & 4 & 6 & 7 & 1 & 0 & 19\end{array}$

* Nursing Home is apparently an ill-defined category. 
Store

Census Division

$1 \quad 2$\begin{tabular}{llll} 
& \multicolumn{3}{l}{ Climate Zones } \\
\end{tabular}

Totals

New England (NE) $18 \quad 103 \quad-\quad-\quad-\quad-$

121

$\begin{array}{lllllllll}\text { Mid-Atlantic } & \text { (NE) } & 8 & 187 & 140 & - & - & -\end{array}$

335

$\begin{array}{lllllllll}\text { So. Atlantic } & (\mathrm{S}) & 2 & 2 & 254 & 342 & 350 & 76 & 1206\end{array}$

E. So. Central (S) $\quad-\quad \begin{array}{lllllll} & - & 78 & 140 & 44 & 73 & 335\end{array}$

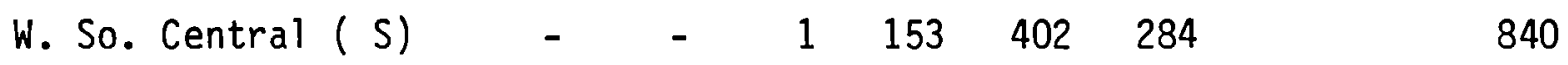

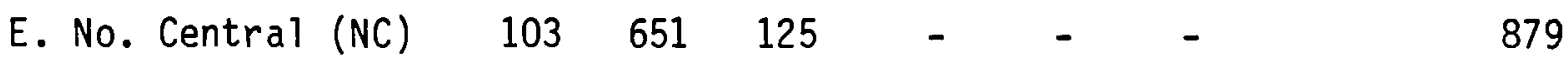

W. No. Central (NC) $\quad \begin{array}{lllllll}128 & 125 & 157 & 0 & - & - & 410\end{array}$

$\begin{array}{lllllllll}\text { Mountain } & (\mathrm{W}) & 39 & 102 & 25 & 18 & 172 & 88 & 444\end{array}$

$\begin{array}{lllllllll}\text { Pacific } & (\mathrm{W}) & 22 & 8 & 79 & 676 & 13 & 106 & 904\end{array}$

Totals

$\begin{array}{llllll}320 & 1178 & 859 & 1329 & 1161 & 627\end{array}$

5474 
Shopping Center

Census Division

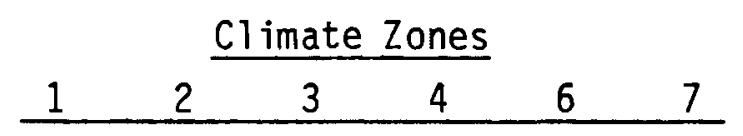

$\underline{\text { Totals }}$

New England (NE) $5 \quad \begin{array}{llllll} & 5 & - & - & - & -\end{array}$

$\begin{array}{llllllll}\text { Mid-Atlantic } & \text { (NE) } & 2 & 34 & 24 & - & - & -\end{array}$

60

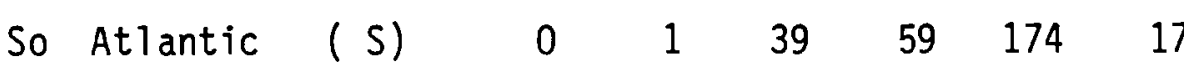

290

E. So. Central (S) - $\quad-\quad \begin{array}{llllll}21 & 30 & 6 & 7 & 64\end{array}$

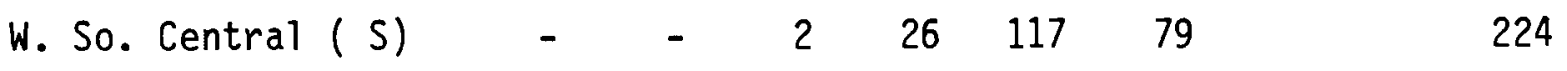

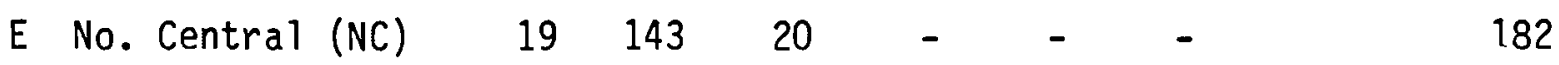

W. No. Central (NC) $\quad \begin{array}{lllllll}11 & 12 & 17 & 0 & - & - & 40\end{array}$

$\begin{array}{lllllllll}\text { Mountain } & (\mathrm{W}) & 15 & 32 & 0 & 7 & 37 & 7 & 98\end{array}$

$\begin{array}{lllllllll}\text { Pacific } & (\mathrm{W}) & 11 & 6 & 23 & 171 & 2 & 18 & 231\end{array}$

Totals

$\begin{array}{llllll}63 & 240 & 146 & 293 & 336 & 128\end{array}$

1206 
Hotel/Motel

Census Division

Climate Zones

$\underline{\text { Totals }}$

\begin{tabular}{llllll}
1 & 2 & 3 & 4 & 6 & 7 \\
\hline
\end{tabular}

New England

$$
\text { (NE) }
$$

$8 \quad 11$

19

$\begin{array}{llllllll}\text { Mid-Atlantic } & \text { (NE) } & 2 & 18 & 9 & - & - & -\end{array}$

29

$\begin{array}{llllllll}\text { So. Atlantic } & (\mathrm{S}) & 3 & 0 & 20 & 17 & 42 & 8\end{array}$

90

E. So. Central ( S) - $\quad$ - $\quad \begin{array}{llllll}4 & 4 & 5 & 2\end{array}$

23

W. So. Central (S) - $\quad \begin{array}{llllll} & - & 0 & 17 & 34 & 30\end{array}$

81

E. No. Central (NC) $14 \quad 41 \quad 8 \quad-\quad$ -

W. No. Central (NC) $\quad \begin{array}{lllllll}18 & 8 & 19 & 0 & - & -\end{array}$

Mounta in

$$
(w)
$$

$18 \quad 2$

$\begin{array}{lllll}21 & 2 & 2 & 15 & 4\end{array}$

62

Pacific

$$
\text { (w) }
$$

2

20

$\begin{array}{lll}57 & 7 & 14\end{array}$

101

Totals

$\begin{array}{llllll}65 & 100 & 82 & 105 & 103 & 58\end{array}$

513 
Warehouse

Census Division

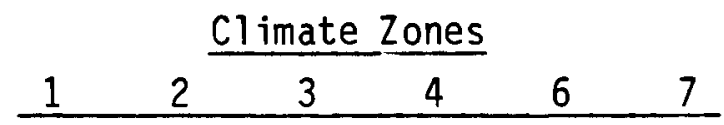

New Engl and (NE) $\quad \begin{array}{llllll}35 & 162 & - & - & - & -\end{array}$

Mid-Atlantic (NE) $\quad 9 \quad 227 \quad 255 \quad-\quad$ - $\quad$ -

491

So. Atlantic $\quad$ ( S) $\quad 4 \quad 4 \quad 1 \quad 317 \quad 466 \quad 1074 \quad 79$

1941

E. So. Central (S) $\quad-\quad \quad-\quad \begin{array}{lllll}S & 111 & 164 & 46 & 35\end{array}$

356

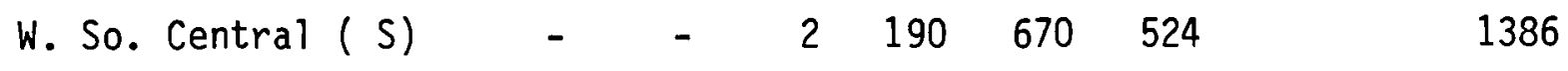

E. No. Central (NC) $\quad \begin{array}{lllllll}190 & 1091 & 215 & - & - & - & 1496\end{array}$

W. No. Central (NC) $\quad 219 \quad 137 \quad 194 \quad 0 \quad-\quad-\quad 550$

$\begin{array}{lllllllll}\text { Mountain } & (W) & 40 & 180 & 46 & 42 & 187 & 38 & 533\end{array}$

$\begin{array}{lllllllll}\text { Pacific } & \text { (W) } & 64 & 10 & 150 & 704 & 44 & 126 & 1098\end{array}$

Totals

$\begin{array}{llllll}561 & 1808 & 1290 & 1566 & 2021 & 802\end{array}$

8048 
Theater/Auditorium

Census Division

Climate Zones

$\underline{\text { Totals }}$

\begin{tabular}{llllll}
1 & 2 & 3 & 4 & 6 & 7 \\
\hline
\end{tabular}

New England

$$
\text { (NE) }
$$

32

5

Mid-Atlantic (NE) $\quad \begin{array}{llllll}0 & 5 & 1 & - & -\end{array}$

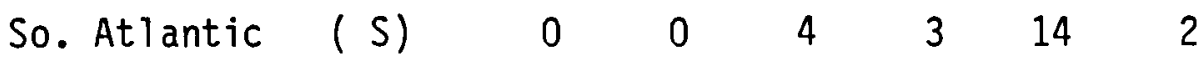

23

E. So. Central ( S) - $\quad-\quad 6 \quad \begin{array}{llll} & 6 & 3\end{array}$

20

W. So. Central ( S) $\quad-\quad \begin{array}{llllll} & 0 & 11 & 14 & 15\end{array}$

40

E. No. Central (NC)

$1 \quad 16 \quad 5 \quad-\quad-\quad-$

22

W. No. Central (NC)

$\begin{array}{llllll}2 & 2 & 7 & 0 & -\end{array}$

11

Mountain

$\begin{array}{lllllll}\text { (W) } & 2 & 4 & 1 & 1 & 4 & 1\end{array}$

13

Pacific

32

816

1

8

38

Totals

$\begin{array}{llllll}11 & 31 & 32 & 42 & 36 & 26\end{array}$

178 
Community Center

Census Division

Climate Zones

$\underline{\text { Totals }}$

\begin{tabular}{llllll}
1 & 2 & 3 & 4 & 6 & 7 \\
\hline
\end{tabular}

New Engl and

(NE)

0

Mid-Atlantic

(NE)

$\begin{array}{lll}0 & 7 & 1\end{array}$

So. Atlantic

$0 \quad 0$

52

13

21

E. So. Central (S) $\quad-\quad \begin{array}{llllll} & 4 & 8 & 0 & 0\end{array}$

12

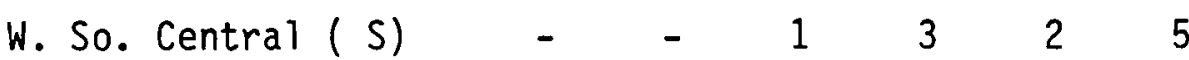

11

E. No. Central (NC) $9 \begin{array}{llllll} & 10 & 3 & - & -\end{array}$

22

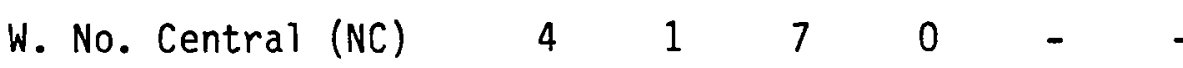

12

Mountain

$\begin{array}{lllllll}\text { (W) } & 7 & 17 & 1 & 2 & 2 & 1\end{array}$

30

Pacific

$\begin{array}{lllllll}\text { (w) } & 13 & 12 & 31 & 42 & 7 & 1\end{array}$

106

Totals

33

48

53

57

24

8

223 
Gymnasium

Census Division

Climate Zones

Totals

\begin{tabular}{llllll}
1 & 2 & 3 & 4 & 6 & 7 \\
\hline
\end{tabular}

New Engl and

(NE)

$\begin{array}{lllll}4 & 18- & - & -\end{array}$

22

Mid-Atlantic (NE) $\quad \begin{array}{llllll} & 0 & 28 & 15 & - & -\end{array}$

43

$\begin{array}{lllllllll}\text { So. Atlantic } & (\mathrm{S}) & 1 & 0 & 27 & 51 & 42 & 6 & 127\end{array}$

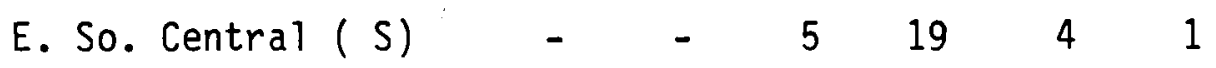

29

W. Co. Central (S) - $\quad-\quad \begin{array}{llllll}S & 1 & 31 & 45 & 52 & 129\end{array}$

E. No. Central (NC) $\quad \begin{array}{lllllll}7 & 50 & 13 & - & - & - & 70\end{array}$

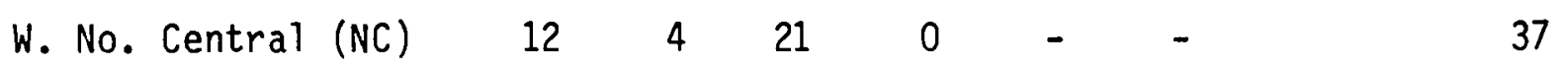

$\begin{array}{lllllllll}\text { Mountain } & (W) & 7 & 17 & 0 & 3 & 9 & 3 & 39\end{array}$

$\begin{array}{lllllllll}\text { Pacific } & (W) & 9 & 8 & 20 & 38 & 2 & 5 & 82\end{array}$

Totals

$\begin{array}{llllll}40 & 125 & 102 & 142 & 102 & 67\end{array}$

578 
No. of

Copies

\section{OFFSITE}

A. A. Churm DOE Patent Division 9800 S. Cass Avenue Argonne, IL 60439

27 DOE Technical Information Center

1 H. Misurie110

W. S. Fleming \& Associates, Inc. Two Metro Plaza

Suite 204 8240 Professional Place Landover, Maryland 20785

1 Gilford Deringer and Company 719 8th Street, S.E. Washington, D.C. 20003

5 R. Menge

Building \& Community Systems Department of Energy Forrestal Bldg. 1000 Independence Avenue Washington, D.C. 20585
No. of

Copies

ONSITE

DOE Richland Operations Office
H. E. Ransom

22 Pacific Northwest Laboratory

W. M. Bowen (5)

D. E. Deonigi

T. J. Foley

J. A. Mahaffey

A. L. Nieves (5)

A. R. 01 sen

T. L. Willke

Publishing Coordination (2)

Technical Information (5) 


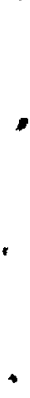

,

$?$

, 